\title{
Crystal and Substituent Effects on Paramagnetic NMR Shifts
}

\section{in Transition-Metal Complexes}

Jan Novotný, ${ }^{\dagger, \$}$ Lukáś Jeremias, ${ }^{\dagger, \$}$ Patrick Nimax, ${ }^{\dagger, \Delta}$ Stanislav Komorovsky, ${ }^{\$}$ Ivo Heinmaa, ${ }^{\mathfrak{R}}$ Radek Marek $* \dagger, \sharp, \Delta$

${ }^{\dagger}$ CEITEC - Central European Institute of Technology, Masaryk University, Kamenice 5, CZ62500 Brno, Czechia

$\$$ Department of Chemistry, Faculty of Science, Masaryk University, Kamenice 5, CZ-625 00 Brno, Czechia

\$Institute of Inorganic Chemistry, Slovak Academy of Sciences, Dúbravská cesta 9, SK-84536 Bratislava, Slovakia

${ }^{\S}$ Department of Chemistry and Biochemistry, Faculty of AgriSciences, Mendel University, Zemědělská 1, CZ-613 00 Brno, Czechia

${ }^{\Delta}$ National Center for Biomolecular Research, Faculty of Science, Masaryk University, Kamenice 5, CZ-625 00 Brno, Czechia

${ }^{£}$ National Institute of Chemical Physics and Biophysics, Akadeemia tee 23, EE-12618 Tallinn, Estonia

\section{Corresponding Author}

*E-mail: radek.marek@ceitec.muni.cz 


\section{SOLID-STATE NMR SPECTROSCOPY}

${ }^{13} \mathrm{C}$ and ${ }^{1} \mathrm{H}$ MAS NMR spectra were recorded on a Bruker AVANCE-II spectrometer at $4.7 \mathrm{~T}$ magnetic field (Larmor frequencies 50.307 and $200.03 \mathrm{MHz}$, for ${ }^{13} \mathrm{C}$ and ${ }^{1} \mathrm{H}$, respectively) using a home-built MAS probe for $1.8 \mathrm{~mm} \mathrm{Si}_{3} \mathrm{~N}_{4}$ rotors. The spectra were recorded with the spinecho pulse sequence $\pi / 2-\tau-\pi-\tau$ - rec, where $\pi / 2=1.8 \mu$ s and $1.5 \mu$ s for ${ }^{13} \mathrm{C}$ and ${ }^{1} \mathrm{H}$, respectively. The ${ }^{13} \mathrm{C}$ NMR spectra were recorded with a $25 \mathrm{~ms}$ relaxation delay between the accumulations and a minimal echo delay of $7 \mu \mathrm{s}$. This caused spinning sidebands to appear out of phase. For ${ }^{1} \mathrm{H}$ resonances the echo delay was one sample rotation period $\tau=\tau_{\mathrm{r}}$. We observed no change in the ${ }^{1} \mathrm{H}$ NMR spectrum when shorter refocusing times were used. ${ }^{1} \mathrm{H}$ NMR spectra were recorded with a $200 \mathrm{~ms}$ relaxation delay and 1200 averages. The chemical shifts are given in TMS scale.

Table S1. NMR parameters for compounds 19 and $2 \mathbf{a}$ obtained by fitting the ${ }^{13} \mathrm{C} \mathrm{CP/MAS}$ spectra in Figure S1 and S2, respectively.

\begin{tabular}{|c|c|c|c|c|}
\hline Temperature $[\mathrm{K}]$ & Parameter & $\mathrm{C} 1$ & $\mathrm{C} 2$ & $\mathrm{C} 3$ \\
\hline \multicolumn{5}{|c|}{ Compound 1a } \\
\hline \multirow[t]{2}{*}{313} & $\delta_{\text {iso }}$ & 1155 & 316 & -421 \\
\hline & (integral) & (153) & $(835)$ & $(1000)$ \\
\hline \multirow[t]{2}{*}{334} & $\delta_{\text {iso }}$ & 1156 & 313 & -397 \\
\hline & (integral) & $(144)$ & $(650)$ & $(1000)$ \\
\hline \multirow[t]{2}{*}{356} & $\delta_{\text {iso }}$ & 937 & 292 & -368 \\
\hline & (integral) & $(108)$ & $(731)$ & $(1000)$ \\
\hline \multicolumn{5}{|c|}{ Compound 2a } \\
\hline \multirow[t]{2}{*}{313} & $\delta_{\text {iso }}$ & 96 & -67 & 929 \\
\hline & (integral) & $(2838)$ & $(1000)$ & (579) \\
\hline \multirow[t]{2}{*}{334} & $\delta_{\text {iso }}$ & 100 & -57 & 841 \\
\hline & (integral) & (2408) & (1000) & (461) \\
\hline \multirow[t]{2}{*}{356} & $\delta_{\text {iso }}$ & 102 & -49 & 800 \\
\hline & (integral) & (2097) & $(1000)$ & $(254)$ \\
\hline
\end{tabular}




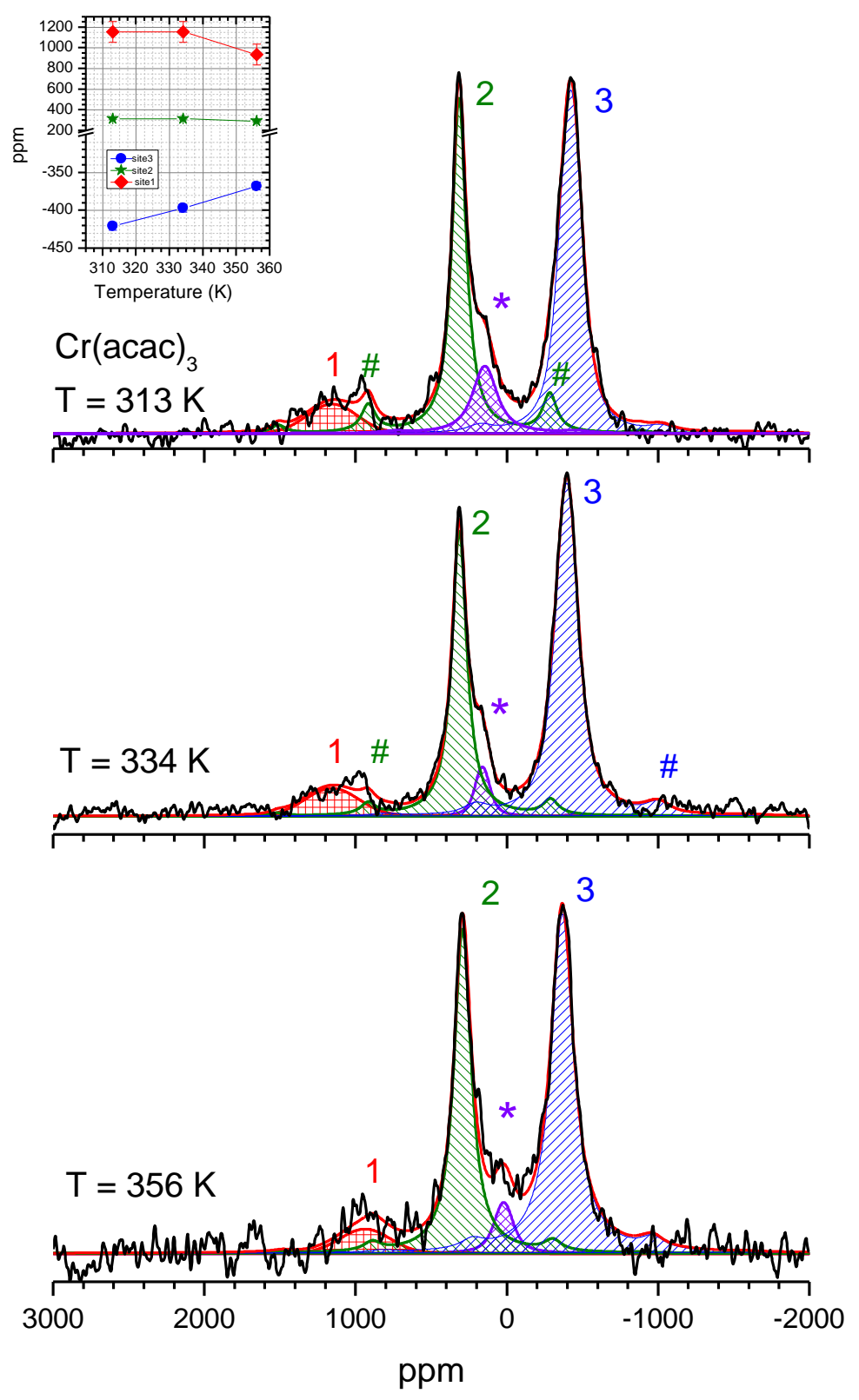

Figure S1. ${ }^{13} \mathrm{C}$ MAS NMR spectrum (30 kHz spinning) of compound $\mathbf{1 a}, \mathrm{Cr}(\mathrm{acac})_{3}$, recorded at $313 \mathrm{~K}$ (top), $334 \mathrm{~K}$ (middle), and $356 \mathrm{~K}$ (bottom). The inset shows the temperature dependence of the three resonances with the estimated error. 

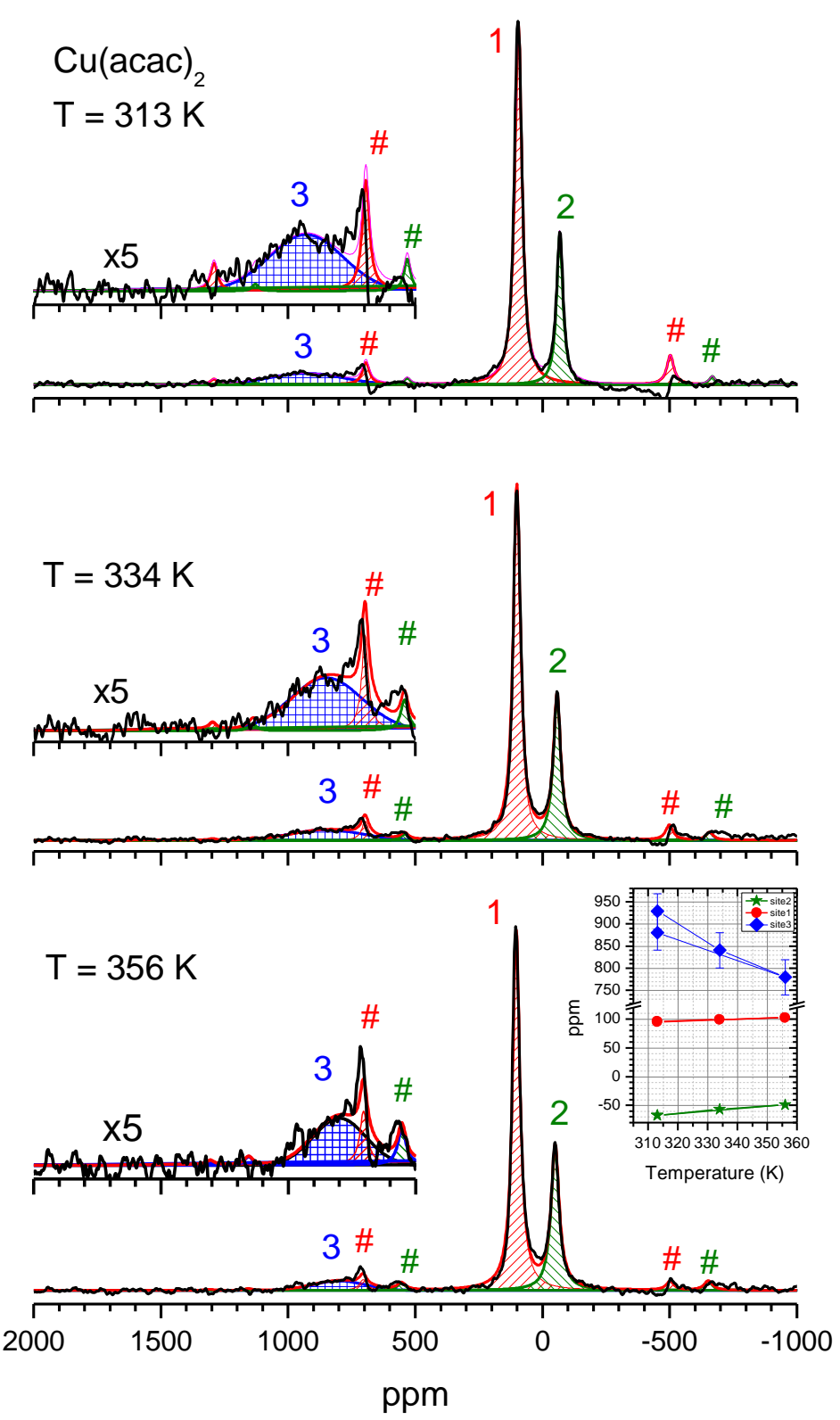

Figure S2. ${ }^{13} \mathrm{C}$ MAS NMR spectrum (30 kHz spinning) of compound $\mathbf{2 a}, \mathrm{Cu}(\mathrm{acac})_{2}$, recorded at $313 \mathrm{~K}$ (top), $334 \mathrm{~K}$ (middle), and $356 \mathrm{~K}$ (bottom). The inset shows the temperature dependence of the three resonances. (The spectrum at $313 \mathrm{~K}$ was recorded two times at different excitation frequencies). Here the scattering of the points gives an estimate of the measurement error of the broad line frequency. 


\section{DFT CALCULATIONS}

Four-component calculations of the NMR and EPR parameters. NMR and EPR parameters were calculated using the program ReSpect (version 5.1.0) ${ }^{1}$ with four-component Dirac-Kohn-Sham (DKS) methodology ${ }^{2-6}$ along with the uncontracted iglo-iii (uiglo-iii) ${ }^{7}$ basis set for the light atoms and Dyall's VTZ basis sets ${ }^{8}$ for the $\mathrm{Cu}, \mathrm{Cr}, \mathrm{Br}$, and I atoms. The calculations were carried out with the PBE0 functional ${ }^{14-15}$ in vacuo (if not stated otherwise).

The value of the temperature-dependent hyperfine shift $\left(\delta_{\mathrm{L}}^{\mathrm{HF}}\right)$ was obtained as the sum of the contributions from the Fermi-contact $\left(\delta_{\mathrm{L}}^{\mathrm{FC}}\right)$, spin-dipole $\left(\delta_{\mathrm{L}}^{\mathrm{SD}}\right)$, and paramagnetic spin-orbit $\left(\delta_{\mathrm{L}}^{\mathrm{PSO}}\right)$ terms, where the separation into $\delta_{\mathrm{L}}^{\mathrm{FC}}, \delta_{\mathrm{L}}^{\mathrm{SD}}$, and $\delta_{\mathrm{L}}^{\mathrm{PSO}}$ shifts is governed by the separation of the hyperfine coupling tensor. ${ }^{12,13}$

All zero-field splitting (ZFS) parameters D and E and the orbital NMR shielding values for compounds $1, \operatorname{Cr}(\mathrm{acac})_{3}$, were taken from the ADF calculations. The ZFS parameters were included in the calculation by using the pNMRShift program. ${ }^{14,15}$ The NMR shielding constants were converted into NMR chemical shifts using the same approach as for the two-component calculations, see main text. 
Table S2. Differences between the hyperfine NMR shifts (in ppm) calculated in the ADF program with and without inclusion of the ZFS parameters ${ }^{a}$ (for details, see Table S3).

\begin{tabular}{|c|c|c|c|}
\hline Atom & 1a & 1d & 2a $-\operatorname{clust}\left(3^{\uparrow \downarrow}\right)$ \\
\hline $\mathbf{C 1}$ & 0.0101 & 0.0131 & 0.3859 \\
\hline $\mathbf{C 2}$ & 0.0067 & 0.0098 & 0.0908 \\
\hline $\mathbf{C 3}$ & 0.0011 & 0.0010 & 0.1624 \\
\hline $\mathbf{H 2}$ & 0.0028 & - & -0.1285 \\
\hline $\mathbf{H 3}$ & 0.0002 & 0.0000 & 0.0147 \\
\hline
\end{tabular}

\section{Geometry optimization}

PBE0/def2-TZVPP/vacuum (Turbomole)

\section{${ }^{a}$ Calculation of NMR shift}

Method 1 (ADF)

a) Orbital shielding: 1c ZORA/PBE0/TZ2P/vacuum

b) $\mathbf{g}$ and A tensors: 2c ZORA/PBE0/TZ2P/vacuum

c) ZFS (D and E values) - 1c ZORA/PBE/TZ2P/vacuum 
Table S3. The orbital $\left(\delta_{\mathrm{L}}^{\mathrm{orb}}\right)$, hyperfine $\left(\delta_{\mathrm{L}}^{\mathrm{HF}}\right)$, and total $\left(\delta_{\mathrm{L}}^{\mathrm{tot}}\right){ }^{13} \mathrm{C}$ and ${ }^{1} \mathrm{H}$ NMR shifts (in ppm) for compounds 1a-e and 2a-e calculated ${ }^{\mathrm{a}}$ in vacuo at $313 \mathrm{~K}$.

\begin{tabular}{|c|c|c|c|c|c|c|c|}
\hline \multirow[t]{2}{*}{ Compound } & & & \multicolumn{5}{|c|}{ NMR Shift [ppm] } \\
\hline & & Atom & $\mathrm{C} 1$ & $\mathrm{C} 2$ & C3 & $\mathrm{H} 2 / \mathrm{C} 4$ & $\mathrm{H} 3$ \\
\hline \multirow{5}{*}{$1 \mathbf{a}$} & \multirow{3}{*}{$\mathrm{ADF}$} & orb & 197.5 & 109.4 & 35.1 & 8.4 & 1.5 \\
\hline & & $\mathrm{HF}$ & 1139.7 & 138.5 & -603.7 & 34.7 & 49.5 \\
\hline & & Tot & 1337.2 & 247.8 & -568.6 & 43.1 & 51.0 \\
\hline & \multirow{2}{*}{ ReSpect } & $\mathrm{HF}$ & 1098.5 & 128.2 & -554.5 & 31.8 & 44.8 \\
\hline & & Tot & 1296.0 & 237.6 & -519.4 & 40.2 & 46.3 \\
\hline \multirow{5}{*}{ 1b } & \multirow{3}{*}{$\mathrm{ADF}$} & orb & 208.0 & 96.9 & 37.7 & 127.8 & 2.0 \\
\hline & & $\mathrm{HF}$ & 1177.7 & 345.3 & -533.8 & 71.4 & 57.2 \\
\hline & & Tot & 1385.7 & 442.2 & -496.1 & 199.2 & 59.2 \\
\hline & \multirow{2}{*}{ ReSpect } & $\mathrm{HF}$ & 1127.8 & 325.2 & -491.7 & 71.5 & 52.1 \\
\hline & & Tot & 1335.8 & 422.1 & -454.0 & 199.4 & 54.1 \\
\hline \multirow{5}{*}{$1 \mathrm{c}$} & \multirow{3}{*}{$\mathrm{ADF}$} & orb & 195.3 & 116.3 & 38.2 & & 2.0 \\
\hline & & $\mathrm{HF}$ & 1177.5 & 796.9 & -513.2 & & 47.1 \\
\hline & & Tot & 1372.8 & 913.2 & -474.9 & & 49.1 \\
\hline & \multirow{2}{*}{ ReSpect } & $\mathrm{HF}$ & 1119.6 & 680.2 & -466.7 & & 46.4 \\
\hline & & Tot & 1314.9 & 796.5 & -428.5 & & 48.3 \\
\hline \multirow{5}{*}{ 1d } & \multirow{3}{*}{$\mathrm{ADF}$} & orb & 199.8 & 123.1 & 40.8 & & 2.0 \\
\hline & & $\mathrm{HF}$ & 1145.2 & 813.5 & -503.6 & & 51.4 \\
\hline & & Tot & 1345.1 & 936.6 & -462.7 & & 53.4 \\
\hline & $\mathrm{PeCnect}$ & $\mathrm{HF}$ & 1101.4 & 785.7 & -463.3 & & 46.9 \\
\hline & Kespect & Tot & 1301.2 & 908.9 & -422.4 & & 48.9 \\
\hline \multirow{5}{*}{$1 e$} & \multirow{3}{*}{$\mathrm{ADF}$} & orb & 202.4 & 108.9 & 40.4 & & 2.0 \\
\hline & & $\mathrm{HF}$ & 1084.3 & 880.0 & -504.3 & & 51.3 \\
\hline & & Tot & 1286.7 & 988.9 & -463.9 & & 53.3 \\
\hline & \multirow{2}{*}{ ReSpect } & $\mathrm{HF}$ & 1046.7 & 852.1 & -465.1 & & 47.3 \\
\hline & & Tot & 1249.1 & 961.0 & -424.7 & & 49.3 \\
\hline & & & & & & & \\
\hline \multirow{6}{*}{$2 \mathbf{a}$} & \multirow{3}{*}{$\mathrm{ADF}$} & orb & 202.7 & 109.7 & 36.4 & 5.5 & 1.3 \\
\hline & & $\mathrm{HF}$ & -65.7 & -126.8 & 1130.1 & -26.9 & -3.4 \\
\hline & & Tot & 136.9 & -17.1 & 1166.6 & -21.4 & -2.1 \\
\hline & & orb & 195.8 & 103.8 & 22.4 & 6.4 & 1.8 \\
\hline & ReSpect & $\mathrm{HF}$ & -50.5 & -128.3 & 1036.3 & -23.3 & -3.1 \\
\hline & & Tot & 145.2 & -24.6 & 1058.7 & $\begin{array}{l}-16.9 \\
\end{array}$ & $\begin{array}{l}-1.3 \\
\end{array}$ \\
\hline & & orb & 209.5 & 98.3 & 38.2 & 125.5 & 1.7 \\
\hline & $\mathrm{ADF}$ & $\mathrm{HF}$ & -158.7 & -153.7 & 1021.9 & -77.6 & -3.9 \\
\hline $2 b$ & & Tot & 50.8 & $\begin{array}{l}-55.4 \\
\end{array}$ & 1060.1 & 47.9 & -2.1 \\
\hline & & orb & 202.9 & 94.7 & 25.0 & 121.2 & 2.4 \\
\hline & ReSpect & $\mathrm{HF}$ & -122.1 & -146.5 & 935.4 & -68.9 & -4.3 \\
\hline & & Tot & 80.9 & -51.7 & 960.5 & 52.2 & -1.9 \\
\hline & & orb & 200.8 & 121.5 & 38.5 & & 1.7 \\
\hline & $\mathrm{ADF}$ & $\mathrm{HF}$ & -47.5 & -253.0 & 993.8 & & -0.6 \\
\hline $2 c$ & & Tot & 153.3 & -131.5 & 1032.3 & & 1.1 \\
\hline & & orb & 193.9 & 113.5 & 24.8 & & 2.3 \\
\hline & ReSpect & $\mathrm{HF}$ & -23.6 & -241.0 & 920.4 & & -1.0 \\
\hline & & Tot & $\begin{array}{l}170.2 \\
\end{array}$ & -127.5 & 945.2 & & 1.3 \\
\hline & & orb & 200.9 & 118.8 & 41.1 & & 1.7 \\
\hline & $\mathrm{ADF}$ & HF & -39.7 & -266.7 & 995.5 & & 0.7 \\
\hline $2 d$ & & Tot & 161.3 & $\begin{array}{l}-147.9 \\
\end{array}$ & 1036.6 & & 2.5 \\
\hline & & orb & 195.2 & 103.2 & 27.5 & & 2.5 \\
\hline & ReSpect & $\mathrm{HF}$ & -23.6 & -254.4 & 915.8 & & -0.1 \\
\hline & & Tot & $\begin{array}{l}171.6 \\
\end{array}$ & -151.2 & 943.3 & & 2.4 \\
\hline $2 \mathrm{e}$ & & orb & 201.5 & 106.2 & 40.4 & & 2.0 \\
\hline $2 \mathrm{e}$ & $\mathrm{ADF}$ & $\mathrm{HF}$ & -28.4 & -256.9 & 1004.5 & & 2.1 \\
\hline & & Tot & \begin{tabular}{|l|}
173.1 \\
\end{tabular} & -150.7 & 1044.9 & & 4.1 \\
\hline
\end{tabular}




\begin{tabular}{|l|r|r|r|r|r|r|r|}
\hline \multirow{3}{*}{ ReSpect } & orb & 197.4 & 73.8 & 32.0 & & 2.7 \\
\cline { 3 - 8 } & & HF & -15.8 & -246.4 & 921.9 & & 0.9 \\
\cline { 3 - 7 } & Tot & $\mathbf{1 8 1 . 6}$ & $-\mathbf{- 1 7 2 . 6}$ & $\mathbf{9 5 3 . 9}$ & & $\mathbf{3 . 6}$ \\
\hline
\end{tabular}

Geometry optimization

PBE0/def2-TZVPP/vacuum (Turbomole)

${ }^{a}$ Calculation of NMR shift

Method 1 (ADF)

a) Orbital shielding: 1c ZORA/PBE0/TZ2P/vacuum

b) $\mathbf{g}$ and A tensors: 2c ZORA/PBE0/TZ2P/vacuum

Method 2 (ReSpect)

a) Orbital shielding: compounds $\mathbf{1}(\mathrm{Cr})$ : from $\mathrm{ADF}$;

compounds $2(\mathrm{Cu})$ : 4c PBE0/VTZ/uiglo-iii/vacuum

b) $\mathbf{g}$ and A tensors: 4c PBE0/VTZ/uiglo-iii/vacuum

Reference

Adamantane $38.48 \mathrm{ppm}\left({ }^{13} \mathrm{C}\right), 1.82 \mathrm{ppm}\left({ }^{1} \mathrm{H}\right)$ 
Table S4. The hyperfine contributions to the ${ }^{13} \mathrm{C}$ and ${ }^{1} \mathrm{H}$ NMR shifts (in ppm) for compounds 1a-e and 2a-e. The values were calculated in the ReSpect program in vacuo at $313 \mathrm{~K}$ (for details, see Table S3).

\begin{tabular}{|c|c|c|c|c|c|c|}
\hline Compound & & & $\mathrm{NM}$ & ift $[\mathrm{ppm}$ & & \\
\hline & Atom & $\mathrm{C} 1$ & $\mathrm{C} 2$ & C3 & $\mathrm{H} 2 / \mathrm{C} 4$ & $\mathrm{H} 3$ \\
\hline \multirow{4}{*}{$1 \mathrm{a}$} & FC & 1104.9 & 128.4 & -554.0 & 31.9 & 44.9 \\
\hline & PSO & -4.3 & 0.0 & -1.6 & -0.2 & -0.1 \\
\hline & SD & 0.1 & 0.1 & 0.0 & 0.0 & 0.0 \\
\hline & HF & 1098.5 & 128.2 & -554.5 & 31.8 & 44.8 \\
\hline \multirow{4}{*}{$1 b$} & FC & 1135.1 & 326.0 & -491.0 & 71.7 & 52.2 \\
\hline & PSO & -5.0 & -0.2 & -1.7 & 0.0 & -0.1 \\
\hline & SD & 0.1 & 0.1 & 0.0 & 0.0 & 0.0 \\
\hline & HF & 1127.8 & 325.2 & -491.7 & 71.5 & 52.1 \\
\hline \multirow{4}{*}{$1 \mathrm{c}$} & FC & 1126.2 & 681.7 & -466.1 & & 46.5 \\
\hline & PSO & -4.4 & -0.2 & -1.6 & & -0.1 \\
\hline & SD & 0.1 & 0.1 & 0.0 & & 0.0 \\
\hline & HF & 1119.6 & 680.2 & -466.7 & & 46.4 \\
\hline \multirow{4}{*}{ 1d } & $\mathrm{FC}$ & 1108.4 & 783.1 & -462.6 & & 47.0 \\
\hline & $\mathrm{PSO}$ & -4.8 & 4.1 & -1.6 & & -0.1 \\
\hline & SD & 0.1 & 0.1 & 0.0 & & 0.0 \\
\hline & HF & 1101.4 & 785.7 & -463.3 & & 46.9 \\
\hline \multirow{4}{*}{$1 \mathrm{e}$} & $\mathrm{FC}$ & 1054.3 & 842.0 & -464.3 & & 47.4 \\
\hline & PSO & -5.5 & 11.8 & -1.7 & & -0.1 \\
\hline & SD & 0.1 & -0.1 & 0.0 & & 0.0 \\
\hline & HF & 1046.7 & 852.1 & -465.1 & & 47.3 \\
\hline \multirow{4}{*}{$2 \mathbf{a}$} & $\mathrm{FC}$ & -44.2 & -125.0 & 1040.0 & -22.0 & -2.1 \\
\hline & PSO & -0.4 & -0.2 & 1.1 & -0.4 & -0.4 \\
\hline & SD & -6.0 & -3.4 & -2.7 & -0.9 & -0.6 \\
\hline & HF & -50.5 & -128.3 & 1036.3 & -23.3 & -3.1 \\
\hline \multirow{4}{*}{$2 b$} & FC & -116.3 & -143.2 & 938.9 & -68.1 & -3.3 \\
\hline & PSO & 0.3 & -0.1 & 1.5 & -0.4 & -0.4 \\
\hline & SD & -6.2 & -3.6 & -3.1 & -0.5 & -0.6 \\
\hline & HF & -122.1 & -146.5 & 935.4 & -68.9 & -4.3 \\
\hline \multirow{4}{*}{$2 c$} & FC & -17.5 & -240.2 & 923.9 & & 0.0 \\
\hline & PSO & 0.1 & 2.3 & 1.3 & & -0.4 \\
\hline & SD & -6.2 & -3.7 & -3.0 & & -0.6 \\
\hline & HF & -23.6 & -241.0 & 920.4 & & -1.0 \\
\hline \multirow{4}{*}{$2 d$} & $\mathrm{FC}$ & -17.6 & -254.7 & 919.2 & & 0.9 \\
\hline & PSO & 0.3 & 3.4 & 1.4 & & -0.4 \\
\hline & SD & -6.2 & -3.7 & -3.0 & & -0.6 \\
\hline & HF & -23.6 & -254.4 & 915.8 & & -0.1 \\
\hline \multirow{4}{*}{$2 e$} & FC & -17.6 & -254.7 & 919.2 & & 0.9 \\
\hline & PSO & 0.5 & 5.3 & 1.6 & & -0.4 \\
\hline & SD & -6.3 & -3.7 & -3.0 & & -0.6 \\
\hline & HF & -15.8 & -246.4 & 921.9 & & 0.9 \\
\hline
\end{tabular}


Table S5. The orbital $\left(\delta_{\mathrm{L}}^{\mathrm{orb}}\right)$, hyperfine $\left(\delta_{\mathrm{L}}^{\mathrm{HF}}\right)$, and total $\left(\delta_{\mathrm{L}}^{\mathrm{tot}}\right){ }^{13} \mathrm{C}$ and ${ }^{1} \mathrm{H}$ NMR shifts (in ppm) for $\mathrm{Cr}(\mathrm{R} \text {-acac })_{3}$ and $\mathrm{Cu}(\mathrm{R} \text {-acac })_{2}$, where $\mathrm{R}=\mathrm{Me}$ or $\mathrm{NO}_{2}$. The values were calculated in vacuo at $313 \mathrm{~K}$ (for details, see Table S3).

\begin{tabular}{|c|c|c|c|c|c|c|c|c|}
\hline Compound & & & \multicolumn{6}{|c|}{ NMR Shift [ppm] } \\
\hline & & Atom & $\mathrm{C} 1$ & $\mathrm{C} 2$ & $\mathrm{C} 3$ & $\mathrm{C} 4$ & $\mathrm{H} 3$ & $\mathrm{H} 4$ \\
\hline \multirow{5}{*}{$\mathrm{Cr}(\mathrm{Me}-\mathrm{acac})_{3}$} & \multirow{3}{*}{$\mathrm{ADF}$} & orb & 198.7 & 110.2 & 38.2 & 26.2 & 2.0 & 1.5 \\
\hline & & $\mathrm{HF}$ & 1146.1 & 429.9 & -589.9 & 90.0 & 45.5 & 49.2 \\
\hline & & Tot & 1344.8 & 540.1 & -551.7 & 116.2 & 47.4 & 50.7 \\
\hline & \multirow{2}{*}{ ReSpect } & $\mathrm{HF}$ & 936.6 & 332.2 & -508.9 & 80.3 & 41.0 & 31.8 \\
\hline & & Tot & 1135.3 & 442.4 & -470.7 & 106.5 & 42.9 & 33.3 \\
\hline \multirow{5}{*}{$\mathrm{Cr}\left(\mathrm{NO}_{2}-\mathrm{acac}\right)_{3}$} & \multirow{3}{*}{$\mathrm{ADF}$} & orb & 201.4 & 145.5 & 40.0 & & & 2.2 \\
\hline & & $\mathrm{HF}$ & 1179.5 & 629.5 & -460.8 & & & 56.8 \\
\hline & & Tot & 1380.9 & 775.1 & -420.8 & & & $\mathbf{5 9 . 0}$ \\
\hline & \multirow{2}{*}{ ReSpect } & $\mathrm{HF}$ & 1127.8 & 596.6 & -425.2 & & & 51.8 \\
\hline & & Tot & 1329.3 & 742.2 & -385.2 & & & 54.0 \\
\hline & & & & & & & & \\
\hline \multirow{6}{*}{$\mathrm{Cu}(\mathrm{Me}-\mathrm{acac})_{2}$} & \multirow{3}{*}{$\mathrm{ADF}$} & orb & 200.0 & 114.4 & 38.8 & 25.6 & 1.8 & 0.9 \\
\hline & & $\mathrm{HF}$ & 21.3 & -146.4 & 1143.6 & -50.2 & -1.3 & 0.0 \\
\hline & & Tot & 221.2 & -31.9 & 1182.4 & -24.6 & 0.4 & 0.8 \\
\hline & \multirow{3}{*}{ ReSpect } & orb & 193.7 & 105.4 & 23.8 & 10.6 & 2.0 & 1.5 \\
\hline & & $\mathrm{HF}$ & 35.1 & -136.1 & 1059.8 & -45.6 & -1.7 & -0.1 \\
\hline & & Tot & 228.8 & -30.7 & 1083.6 & -35.1 & 0.3 & 1.4 \\
\hline \multirow{6}{*}{$\mathrm{Cu}\left(\mathrm{NO}_{2}-\mathrm{acac}\right)_{2}$} & \multirow{3}{*}{$\mathrm{ADF}$} & orb & 203.5 & 147.3 & 36.2 & & 2.0 & \\
\hline & & $\mathrm{HF}$ & -106.4 & -168.7 & 932.8 & & 3.8 & \\
\hline & & Tot & 97.1 & -21.4 & 969.0 & & 5.9 & \\
\hline & \multirow{3}{*}{ ReSpect } & orb & 198.3 & 143.8 & 25.0 & & 2.4 & \\
\hline & & $\mathrm{HF}$ & -91.5 & -162.7 & 858.2 & & 2.9 & \\
\hline & & Tot & 106.8 & -18.8 & 883.2 & & 5.3 & \\
\hline
\end{tabular}

Table S6. The hyperfine contributions to the ${ }^{13} \mathrm{C}$ and ${ }^{1} \mathrm{H} N M R$ shifts for $\mathrm{Cr}(\mathrm{R}-\mathrm{acac})_{3}$ and $\mathrm{Cu}(\mathrm{R}-$ acac) 2 , where $\mathrm{R}=\mathrm{Me}$ or $\mathrm{NO}_{2}$. The values were calculated in the ReSpect program in vacuo at $313 \mathrm{~K}$ (for details, see Table S3).

\begin{tabular}{|c|c|c|c|c|c|c|c|}
\hline \multirow[t]{2}{*}{ Compound } & & \multicolumn{6}{|c|}{ NMR Shift [ppm] } \\
\hline & Atom & $\mathrm{C} 1$ & $\mathrm{C} 2$ & $\mathrm{C} 3$ & $\mathrm{C} 4$ & $\mathrm{H} 3$ & $\mathrm{H} 4$ \\
\hline \multirow{4}{*}{$\mathrm{Cr}(\mathrm{Me}-\mathrm{acac})_{3}$} & $\mathrm{FC}$ & 942.6 & 333.5 & -508.5 & 80.4 & 41.1 & 31.8 \\
\hline & PSO & -4.2 & -0.7 & -1.4 & 0.0 & -0.1 & 0.0 \\
\hline & SD & 0.1 & 0.1 & 0.0 & 0.0 & 0.0 & 0.0 \\
\hline & HF & 936.6 & 332.2 & -508.9 & 80.3 & 41.0 & 31.8 \\
\hline \multirow{4}{*}{$\mathrm{Cr}\left(\mathrm{NO}_{2}-\mathrm{acac}\right)_{3}$} & $\mathrm{FC}$ & 1135.4 & 598.5 & -424.3 & & & 51.9 \\
\hline & PSO & -5.3 & -0.8 & -1.8 & & & -0.1 \\
\hline & SD & 0.1 & 0.1 & 0.0 & & & 0.0 \\
\hline & HF & 1127.8 & 596.6 & -425.2 & & & 51.8 \\
\hline \multirow{4}{*}{$\mathrm{Cu}(\mathrm{Me}-\mathrm{acac})_{2}$} & $\mathrm{FC}$ & 41.6 & -133.5 & 1063.7 & -44.7 & -0.7 & 0.6 \\
\hline & PSO & -0.4 & 0.4 & 1.2 & -0.5 & -0.4 & -0.3 \\
\hline & SD & -6.0 & -3.3 & -3.0 & -0.5 & -0.6 & -0.4 \\
\hline & HF & 35.1 & -136.1 & 1059.8 & -45.6 & -1.7 & -0.1 \\
\hline \multirow{4}{*}{$\mathrm{Cu}\left(\mathrm{NO}_{2}-\mathrm{acac}\right)_{2}$} & $\mathrm{FC}$ & -85.8 & -160.1 & 861.4 & & 3.9 & \\
\hline & PSO & 0.6 & 0.5 & 1.5 & & -0.4 & \\
\hline & SD & -6.4 & -3.5 & -2.9 & & -0.6 & \\
\hline & HF & -91.5 & -162.7 & 858.2 & & 2.9 & \\
\hline
\end{tabular}


Table S7. Hyperfine coupling constants $\left(A_{\text {iso }}\right)$ and hyperfine NMR shifts $(\mathrm{T}=313 \mathrm{~K})$ for compounds $1 \mathbf{a}$ and $1 \mathbf{d}$ calculated ${ }^{\mathrm{a}}$ at the $1 \mathrm{c}$ and $2 \mathrm{c}$ levels of theory.

\begin{tabular}{|c|c|c|c|c|}
\hline \multirow{3}{*}{ Atom } & \multicolumn{4}{|c|}{$1 a$} \\
\hline & \multicolumn{2}{|c|}{$A_{\text {iso }}[\mathrm{MHz}]$} & \multicolumn{2}{|c|}{ Total NMR shift [ppm] } \\
\hline & $2 \mathrm{c}$ & $1 \mathrm{c}$ & $2 c$ & $1 \mathrm{c}$ \\
\hline $\mathrm{C} 1$ & 2.295 & 2.290 & 1337.2 & 1335.0 \\
\hline $\mathrm{C} 2$ & 0.279 & 0.297 & 247.8 & 256.8 \\
\hline $\mathrm{C} 3$ & -1.216 & -1.214 & -568.6 & -567.8 \\
\hline $\mathrm{H} 2$ & 0.278 & 0.280 & 43.1 & 43.4 \\
\hline \multirow[t]{3}{*}{$\mathrm{H} 3$} & 0.396 & 0.396 & 51.0 & 51.0 \\
\hline & \multicolumn{4}{|c|}{ 1d } \\
\hline & $2 c$ & $1 \mathrm{c}$ & $2 c$ & $1 \mathrm{c}$ \\
\hline $\mathrm{C} 1$ & 2.306 & 2.290 & 1345.0 & 1337.3 \\
\hline $\mathrm{C} 2$ & 1.638 & 1.652 & 936.6 & 943.6 \\
\hline $\mathrm{C} 3$ & -1.014 & -1.009 & -462.7 & -460.0 \\
\hline $\mathrm{H} 3$ & 0.412 & 0.412 & 53.4 & 53.4 \\
\hline
\end{tabular}

\section{Geometry optimization}

PBE0/def2-TZVPP/vacuum (Turbomole)

\section{${ }^{\text {a }}$ Calculation of NMR shift}

a) Orbital shielding: 1c ZORA/PBE0/TZ2P/vacuum

b) g-tensor: 2c ZORA/PBE0/TZ2P/vacuum

c) A-tensors: 1c ZORA/PBE0/TZ2P/vacuum

Reference

Adamantane $38.48 \mathrm{ppm}\left({ }^{13} \mathrm{C}\right), 1.82 \mathrm{ppm}\left({ }^{1} \mathrm{H}\right)$ 
Table S8. The effect of the exact-exchange admixture in the PBE-based functional on the total NMR shift of compounds $\mathbf{1 a}$ and $\mathbf{2 a}$ in $\mathrm{CHCl}_{3}$ at $313 \mathrm{~K}$.

\begin{tabular}{|c|c|c|c|c|c|}
\hline \multirow{2}{*}{$\begin{array}{l}\text { Functional } \\
\qquad 1 \mathbf{a}\end{array}$} & \multicolumn{5}{|c|}{ NMR Shift [ppm] } \\
\hline & $\mathrm{C} 1$ & $\mathrm{C} 2$ & $\mathrm{C} 3$ & $\mathrm{H} 2$ & H3 \\
\hline PBE & 1741.0 & 222.6 & -696.3 & 62.8 & 107.2 \\
\hline PBE0-05 & 1651.3 & 231.7 & -681.3 & 58.7 & 89.5 \\
\hline PBE0-10 & 1564.9 & 229.6 & -646.9 & 52.9 & 76.5 \\
\hline PBE0-15 & 1483.1 & 228.8 & -615.3 & 48.4 & 66.2 \\
\hline PBE0-20 & 1414.1 & 226.8 & -582.3 & 44.4 & 58.2 \\
\hline PBEO & 1354.7 & 225.4 & -550.6 & 40.9 & 51.6 \\
\hline PBE0-30 & 1296.3 & 218.9 & -518.2 & 38.3 & 46.2 \\
\hline PBE0-35 & 1253.8 & 224.1 & -491.1 & 35.0 & 41.9 \\
\hline PBE0-40 & 1204.9 & 219.6 & -465.6 & 32.1 & 38.3 \\
\hline $2 \mathbf{a}$ & $\mathrm{C} 1$ & $\mathrm{C} 2$ & $\mathrm{C} 3$ & $\mathrm{H} 2$ & $\mathrm{H} 3$ \\
\hline PBE & 13.2 & -4.9 & 1658.6 & -28.0 & 1.9 \\
\hline PBE0-05 & 25.4 & -16.1 & 1570.8 & -27.6 & 1.6 \\
\hline PBE0-10 & 34.9 & -22.2 & 1447.5 & -26.3 & 0.9 \\
\hline PBE0-15 & 45.3 & -21.8 & 1328.6 & -24.6 & 0.2 \\
\hline PBE0-20 & 99.0 & -17.2 & 1217.0 & -22.7 & -0.6 \\
\hline PBEO & 121.6 & -11.3 & 1111.4 & -21.5 & -1.2 \\
\hline PBE0-30 & 140.7 & -2.0 & 1013.7 & -19.2 & -1.8 \\
\hline PBE0-35 & 159.9 & 5.8 & 925.1 & -17.6 & -1.9 \\
\hline PBE0-40 & 175.2 & 15.2 & 846.2 & -16.0 & -2.2 \\
\hline PBE0-45 & 188.7 & 23.1 & 777.8 & -15.4 & -3.0 \\
\hline PBE0-50 & 193.4 & 31.3 & 719.7 & -14.3 & -3.3 \\
\hline
\end{tabular}

\section{Geometry optimization}

PBE0/def2-TZVPP/CHCl 3 (Turbomole)

\section{Calculation of NMR shift}

a) Orbital shielding: 1c ZORA/PBE0-X/TZ2P/CHCl

b) g- and A-tensors: 2c ZORA/PBE0-X/TZ2P/CHCl 3 


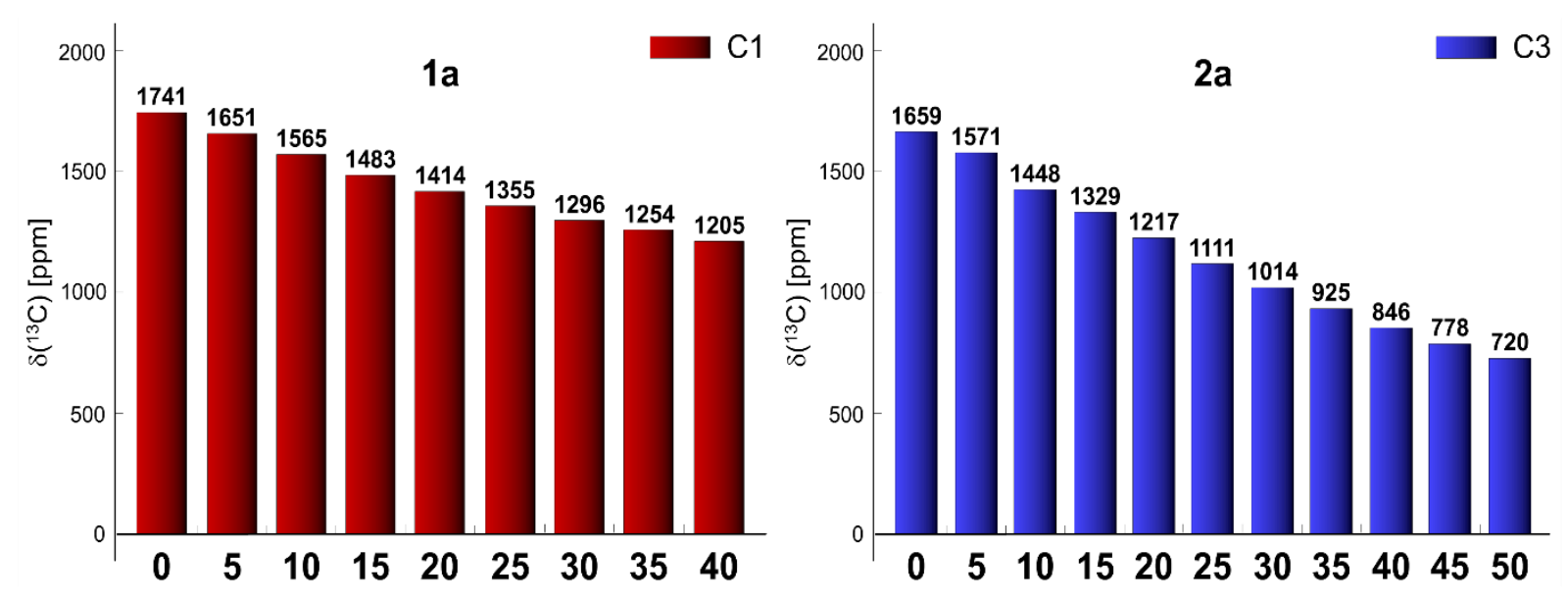

Figure S3. The effect of the exact-exchange admixture in the PBE-based functional on the total NMR shift of $\mathrm{C} 1$ in compound $\mathbf{1 a}$ (left) and C3 in compound 2a (right) in chloroform at 313 K. For the similar dependence for C2 in Ru(III) compounds, see Ref. 16. 


\section{SYNTHESIS}

Solvents used during the preparation of the compounds were of p.a. grade. $\mathrm{CH}_{2} \mathrm{Cl}_{2}, \mathrm{CHCl}_{3}$, and benzene were distilled over $\mathrm{Na}_{2} \mathrm{SO}_{4}$ and stored under mol sieves ( $4 \AA$ ) for $24 \mathrm{~h}$ before use. DMF was dried over $\mathrm{MgSO}_{4}$, distilled under reduced pressure, and stored over mol sieves. Pentane2,4-dione was purchased from Penta, distilled over $\mathrm{MgSO}_{4}$, and stored over mol sieves prior to use. $\mathrm{ClSO}_{2} \mathrm{NCO}$ was purchased from Sigma Aldrich, 3-chloropentane-2,4-dione was purchased from TCI and used as is. Unless stated otherwise, no protective gas was used during the preparation of the compounds.

\section{Ligands}

Preparation of compound L1. Compound L1 (3-cyanopentane-2,4-dione) was prepared according to Silvernail et al. ${ }^{17} \mathrm{~A}$ hot, $100 \mathrm{~mL}$ flask was sealed with a septum and purged under heating three times with argon. It was charged with $27 \mathrm{~mL}$ of $\mathrm{CH}_{2} \mathrm{Cl}_{2}$ and 2,4-pentanedione $(10.0 \mathrm{~g}, 0.100 \mathrm{~mol})$. Under vigorous stirring, $\mathrm{ClSO}_{2} \mathrm{NCO}(4.75 \mathrm{~mL}, 7.72 \mathrm{~g}, 0.055 \mathrm{~mol})$ was added dropwise over a period of $5 \mathrm{~min}$ and stirred for an additional $5 \mathrm{~min}$ before DMF was added $(8.40 \mathrm{~mL}, 0.108 \mathrm{~mol})$. The solution was stirred at room temperature for $30 \mathrm{~min}$, washed with $\mathrm{H}_{2} \mathrm{O}(2 \times 50 \mathrm{~mL})$, and dried over $\mathrm{Na}_{2} \mathrm{SO}_{4}$. The solvent was removed in vacuo and the residue was taken up in $2 \mathrm{~mL}$ of $\mathrm{CH}_{2} \mathrm{Cl}_{2}$ and stored at $-5^{\circ} \mathrm{C}$ overnight in order to crystallize. The red needles were washed with $\mathrm{H}_{2} \mathrm{O}$ and dried in vacuo. Yield: $4.3 \mathrm{~g}(34.4 \%)$.

Analysis:

MS, m/z (ESI-): $124.04\left(\left(\text { acac-CN)-H }{ }^{+}\right)^{-}\right)$.

\section{Compound 1}

Preparation of compound 1a - $\mathbf{C r}(\mathbf{a c a c})_{3}$. Compound 1a was prepared using a variation of Wang et al. ${ }^{18}$ Pentane-2,4-dione $(0.76 \mathrm{~mL}, 7.5 \mathrm{mmol})$ was added to $\mathrm{CrCl}_{3} \cdot 6 \mathrm{H}_{2} \mathrm{O}(0.67 \mathrm{~g}, 2.5$ $\mathrm{mmol})$ in $\mathrm{H}_{2} \mathrm{O}$ and urea $(0.45 \mathrm{mmol})$, and the solution was stirred at reflux for $5 \mathrm{~h}$. The solvent was partially removed in vacuo, and the purple, crystalline precipitate was isolated by filtration and dried in vacuo. Yield: $0.3 \mathrm{~g}(34 \%)$.

Analysis:

MS, m/z (ESI+): $350.08\left(\left[\mathrm{Cr}(\mathrm{acac})_{3}\right]+\mathrm{H}^{+}\right)$.

${ }^{1} \mathrm{H} \mathrm{NMR}\left(\mathrm{CDCl}_{3}, 298 \mathrm{~K}\right), \delta:+28.7 \mathrm{ppm}(\mathrm{H} 2, \mathrm{CH}),+41.0 \mathrm{ppm}\left(\mathrm{H} 3, \mathrm{CH}_{3}\right)$.

${ }^{1} \mathrm{H}$ NMR (solid state, $\left.313 \mathrm{~K}\right), \delta:+40.8 \mathrm{ppm}\left(\mathrm{H} 3, \mathrm{CH}_{3}\right)$.

Preparation of compound 1b - $\mathbf{C r}(\mathbf{a c a c C N}) 3.3-C y a n o p e n t a n e-2,4-d i o n e(\mathbf{L 1}, 0.94 \mathrm{~g}$, $7.5 \mathrm{mmol})$ was added to $\mathrm{CrCl}_{3} \cdot 6 \mathrm{H}_{2} \mathrm{O}(0.67 \mathrm{~g}, 2.5 \mathrm{mmol})$ and urea $(0.45 \mathrm{~g}, 2.5 \mathrm{mmol})$ in a mixture of $\mathrm{H}_{2} \mathrm{O} / \mathrm{EtOH}(1: 1,50 \mathrm{~mL})$. The solution was refluxed for $18 \mathrm{~h}$, the solvent was extracted with Brine/DCM, and the organic phase was washed with water and dried over $\mathrm{MgSO}_{4}$. Column chromatography (silica, DCM) afforded the product as a brown powder that was dried in vacuo and recrystallized from benzene/ $\mathrm{CHCl}_{3}$. Yield: $0.58 \mathrm{~g}(54 \%)$.

Analysis:

${ }^{1} \mathrm{H}$ NMR $\left(\mathrm{CDCl}_{3}, 313 \mathrm{~K}\right), \delta:+44.4 \mathrm{ppm}\left(\mathrm{H} 3, \mathrm{CH}_{3}\right)$.

IR, $v\left(\mathrm{~cm}^{-1}\right): 2923.5,2207.3,1732.0,1563.5,1342.0,1016.6,799.5,631.3,453.0$. 
Preparation of compound $1 \mathrm{c}-\mathbf{C r}(\operatorname{acacCl})_{3}$. Compound $\mathbf{1 c}$ was prepared using a variation of Muena et al. ${ }^{19}$ 3-Chloropentane-2,4-dione $(3.71 \mathrm{~mL}, 16.5 \mathrm{mmol})$ was added to $\mathrm{CrCl}_{3} \cdot 6 \mathrm{H}_{2} \mathrm{O}$ $(1.48 \mathrm{~g}, 5.5 \mathrm{mmol})$ and urea $(1.0 \mathrm{~g}, 5.5 \mathrm{mmol})$ in a mixture of $\mathrm{H}_{2} \mathrm{O} / \mathrm{EtOH}(1: 1,50 \mathrm{~mL})$. The solution was stirred at RT for $3 \mathrm{~h}$, and then stirred at reflux for an additional $2 \mathrm{~h}$. The brown precipitate was isolated by filtration, washed with water and $\mathrm{Et}_{2} \mathrm{O}$, and dried in vacuo. Yield: $1.8 \mathrm{~g}(72 \%)$.

Analysis:

${ }^{1} \mathrm{H}$ NMR $\left(\mathrm{CDCl}_{3}, 313 \mathrm{~K}\right), \delta:+38.9 \mathrm{ppm}\left(\mathrm{H} 3, \mathrm{CH}_{3}\right)$.

IR, $v\left(\mathrm{~cm}^{-1}\right): 3337.7,3013.3,2924.3,1561.5,1378.5,1017.3,625.6,461.7$.

Preparation of compound 1d - $\mathbf{C r}(\mathbf{a c a c B r}) 3$. Compound 1d was prepared according to Collman et al..$^{20}$ In a flame-dried flask, NBS $(0.4 \mathrm{~g}, 0.023 \mathrm{~mol})$ was dissolved in anhydrous $\mathrm{CHCl}_{3}(10 \mathrm{~mL})$ and compound $\mathbf{1 a}(0.5 \mathrm{~g}, 1.44 \mathrm{mmol})$ dissolved in $\mathrm{CHCl}_{3}(10 \mathrm{~mL})$ was added dropwise under stirring at RT over a period of $5 \mathrm{~min}$. The solution was stirred for $20 \mathrm{~min}$, during which time a precipitate formed. This was washed with water $(5 \mathrm{~mL}), \mathrm{NaHCO}_{3}$ solution $(5 \mathrm{~mL})$, water $(5 \mathrm{~mL})$, and $\mathrm{Et}_{2} \mathrm{O}(30 \mathrm{~mL})$, to yield a brown powder that was dried in vacuo. Yield: $0.6 \mathrm{~g}$ $(71 \%)$.

Analysis:

${ }^{1} \mathrm{H} \mathrm{NMR}\left(\mathrm{CDCl}_{3}, 313 \mathrm{~K}\right), \delta:+38.1 \mathrm{ppm}\left(\mathrm{H} 3, \mathrm{CH}_{3}\right)$.

IR, $v\left(\mathrm{~cm}^{-1}\right): 1546.2,1327.5,1020.8,752.18,608.8,466.4$.

\section{Compound 2}

Preparation of compound 2a - Cu(acac) 2. After $\mathrm{Cu}\left(\mathrm{NO}_{3}\right)_{2} \cdot 3 \mathrm{H}_{2} \mathrm{O}(10 \mathrm{~g}, 41.4 \mathrm{mmol})$ in $\mathrm{NH}_{3}$ (aq., $25 \mathrm{~mL}$ ) had been stirred for $15 \mathrm{~min}$, pentane-2,4-dione $(11 \mathrm{~mL}, 107 \mathrm{mmol})$ was added dropwise. The solution was then stirred for $10 \mathrm{~min}$ at RT. The blue precipitate was isolated by filtration, washed with $\mathrm{H}_{2} \mathrm{O}(3 \times 50 \mathrm{~mL})$ and cold $\mathrm{DCM}(20 \mathrm{~mL})$, and dried in vacuo. Recrystallization from DCM afforded blue needles of 2a. Yield:6.3 g (58\%).

Analysis:

MS, m/z (ESI+): $262.02\left(\left[\mathrm{Cu}(\mathrm{acac})_{2}\right]+\mathrm{H}^{+}\right), 423.0\left(\left[\mathrm{Cu}_{2}(\mathrm{acac})_{3}\right]^{+}\right)$.

${ }^{1} \mathrm{H} \mathrm{NMR}\left(\mathrm{CDCl}_{3}, 293 \mathrm{~K}\right), \delta:+1.0 \mathrm{ppm}\left(\mathrm{H} 3, \mathrm{CH}_{3}\right),-22.1 \mathrm{ppm}(\mathrm{H} 2, \mathrm{CH})$.

${ }^{1} \mathrm{H}$ NMR (solid state, $\left.313 \mathrm{~K}\right), \delta:+1.8 \mathrm{ppm}\left(\mathrm{H} 3, \mathrm{CH}_{3}\right),-8.6 \mathrm{ppm}(\mathrm{H} 2, \mathrm{CH})$

Preparation of compound $2 \mathbf{b}-\mathbf{C u}(\mathbf{a c a c C N})_{2}$. Compound $2 \mathrm{~b}$ was prepared according to Silvernail et al. ${ }^{17}$ 3-Cyanopentane-2,4-dione (L1, $\left.0.62 \mathrm{~g}, \quad 5 \mathrm{mmol}\right)$ was added to $\mathrm{Cu}\left(\mathrm{NO}_{3}\right)_{2} \times 3 \mathrm{H}_{2} \mathrm{O}(0.60 \mathrm{~g}, 2.5 \mathrm{mmol})$ in a mixture of $\mathrm{EtOH} / \mathrm{H}_{2} \mathrm{O}(1: 1,50 \mathrm{~mL})$. The solution was stirred for $2 \mathrm{~h}$ at $\mathrm{RT}$, and the blue precipitate was isolated by filtration and dried in vacuo.

Yield: $0.6 \mathrm{~g}(77 \%)$.

Analysis:

MS, m/z (ESI-): 435.05 ([Cu(acac-CN $\left.\left.)_{3}\right]^{-}\right)$.

${ }^{1} \mathrm{H}$ NMR (methanol- $\left.d_{4}, 313 \mathrm{~K}\right), \delta:+2.4 \mathrm{ppm}\left(\mathrm{H} 3, \mathrm{CH}_{3}\right)$.

${ }^{1} \mathrm{H}$ NMR (solid state, $\left.313 \mathrm{~K}\right), \delta:+6.2 \mathrm{ppm}\left(\mathrm{H} 3, \mathrm{CH}_{3}\right)$.

IR, $v\left(\mathrm{~cm}^{-1}\right): 3491.3,2926.8,2194,1590.8,1394.1,1001.1,638.97,447.65$. 
Preparation of compound $2 \mathbf{c}-\mathbf{C u}(\mathbf{a c a c C l}) 2$. Compound $2 \mathbf{c}$ was prepared using a variation of Muena et al. ${ }^{19}$ 3-Chloropentane-2,4-dione $(2.24 \mathrm{~mL}, 10 \mathrm{mmol})$ was added to $\mathrm{Cu}\left(\mathrm{NO}_{3}\right)_{2} \times 3 \mathrm{H}_{2} \mathrm{O}(1.2 \mathrm{~g}, 5 \mathrm{mmol})$ and urea $(0.9 \mathrm{~g}, 5 \mathrm{mmol})$ in $\mathrm{EtOH} / \mathrm{H}_{2} \mathrm{O}(1: 1,50 \mathrm{~mL})$. The solution was stirred for $3 \mathrm{~h}$ at RT, and the green precipitate was isolated by filtration and dried in vacuo. Recrystallisation from benzene/hexane afforded green needles. Yield: $1.3 \mathrm{~g}$ (78\%).

Analysis:

MS, m/z (ESI+): $526.87\left(\left[\mathrm{Cu}_{2}(\text { acac-Cl })_{3}\right]^{+}\right)$.

${ }^{1} \mathrm{H} \mathrm{NMR}\left(\mathrm{CDCl}_{3}, 313 \mathrm{~K}\right), \delta:+6.2 \mathrm{ppm}\left(\mathrm{H} 3, \mathrm{CH}_{3}\right)$.

${ }^{1} \mathrm{H}$ NMR (solid state, $\left.313 \mathrm{~K}\right), \delta:+7.2 \mathrm{ppm}\left(\mathrm{H} 3, \mathrm{CH}_{3}\right)$.

IR, $v\left(\mathrm{~cm}^{-1}\right): 3337.7,3013.3,2924.3,1561.5,1378.5,1017.3,625.6,461.7$.

Preparation of compound 2d - $\mathbf{C u}(\mathbf{a c a c B r}) 2$. Compound 2d was prepared according to Collman et al. ${ }^{20}$ In a flame-dried flask, NBS $(0.4 \mathrm{~g}, 0.023 \mathrm{~mol})$ was dissolved in anhydrous $\mathrm{CHCl}_{3}(10 \mathrm{~mL})$ and compound $\mathbf{2 a}(0.57 \mathrm{~g}, 2.1 \mathrm{mmol})$ dissolved in $\mathrm{CHCl}_{3}(10 \mathrm{~mL})$ was added dropwise under stirring at RT over a period of $5 \mathrm{~min}$. The solution was stirred for $20 \mathrm{~min}$, during which time the solution changed color from blue to green. The solvent was removed in vacuo, and the product was washed with water $(5 \mathrm{~mL}), \mathrm{NaHCO}_{3}$ solution $(5 \mathrm{~mL})$, water $(5 \mathrm{~mL})$, and $\mathrm{Et}_{2} \mathrm{O}(30 \mathrm{~mL})$, to yield a green powder that was recrystallized from benzene/hexane and dried in vacuo. Yield: $0.64 \mathrm{~g}(72 \%)$.

Analysis:

MS, m/z (ESI+): 502.90 ([Cu(acac-Br)(acacH-Br) $\left.]^{+}+\mathrm{HBr}\right)$.

${ }^{1} \mathrm{H} \mathrm{NMR}, \delta\left(\mathrm{CDCl}_{3}, 308 \mathrm{~K}\right):+7.1 \mathrm{ppm}\left(\mathrm{H} 3, \mathrm{CH}_{3}\right)$.

${ }^{1} \mathrm{H}$ NMR, $\delta($ solid state, $313 \mathrm{~K}):+8.0 \mathrm{ppm}\left(\mathrm{H} 3, \mathrm{CH}_{3}\right)$.

IR, $v\left(\mathrm{~cm}^{-1}\right): 3297.7,3117.9,3012.1,2925.7,2811.4,2311.5,2115,1703.9,1559.3,1375.9$, 1019.3, 775.36, 630.51, 463.45.

Preparation of $\mathrm{Cu}(\mathbf{a c a c I})$ (acac). This compound was prepared according to Collman et al. ${ }^{20}$ In a flame-dried flask, NIS $(0.41 \mathrm{~g}, 0.02 \mathrm{~mol})$ was dissolved in anhydrous $\mathrm{CHCl}_{3}(10 \mathrm{~mL})$ and compound 2a $(0.5 \mathrm{~g}, 1.84 \mathrm{mmol})$ dissolved in $\mathrm{CHCl}_{3}(10 \mathrm{~mL})$ was added dropwise under stirring at RT over a period of $5 \mathrm{~min}$. The solution was stirred for $20 \mathrm{~min}$, during which time it changed color from blue to green. The solvent was removed in vacuo, and the product was washed with water $(5 \mathrm{~mL}), \mathrm{NaHCO}_{3}$ solution $(5 \mathrm{~mL})$, water $(5 \mathrm{~mL})$, and $\mathrm{Et}_{2} \mathrm{O}(30 \mathrm{~mL})$, to yield a green powder that was recrystallized from benzene/hexane and dried in vacuo. Yield: $0.78 \mathrm{~g}$ (82\%).

Analysis:

MS, m/z (ESI+): $387.93\left([\mathrm{Cu}(\text { acac-I })(\text { acac })]^{+}\right) ; 513.82\left([\mathrm{Cu}(\text { acac-I })(\text { acacH-I })]^{+}\right)$.

${ }^{1} \mathrm{H}$ NMR $\left(\mathrm{CDCl}_{3}, 313 \mathrm{~K}\right), \delta:+8.1 \mathrm{ppm}(\mathrm{H} 3$, acac-I), +0.9 ppm (H3, acac), -21.4 ppm (H2, acac).

${ }^{1} \mathrm{H}$ NMR (solid state, $\left.313 \mathrm{~K}\right), \delta:+9.2 \mathrm{ppm}(\mathrm{H} 3$, acac-I), +1.1 ppm (H3, acac), -9.0 ppm (H2, acac).

IR, $v\left(\mathrm{~cm}^{-1}\right): 2917.8,1547.5,1408,1015.3,801.08,630,458.64$. 


\section{REFEFERENCES}

(1) Repisky, M.; Komorovsky, S.; Kadek, M.; Konecny, L.; Ekström, U.; Malkin, E.; Kaupp, M.; Ruud, K.; Malkina, O. L.; Malkin, V. G. ReSpect: Relativistic Spectroscopy DFT Program Package. J. Chem. Phys. 2020, 152 (18), 184101. https://doi.org/10.1063/5.0005094.

(2) Repiský, M.; Komorovský, S.; Malkin, E.; Malkina, O. L.; Malkin, V. G. Relativistic Four-Component Calculations of Electronic g-Tensors in the Matrix Dirac-KohnSham Framework. Chem. Phys. Lett. 2010, 488 (1-3), 94-97. https://doi.org/10.1016/j.cplett.2010.01.077.

(3) Gohr, S.; Hrobárik, P.; Repiský, M.; Komorovský, S.; Ruud, K.; Kaupp, M. FourComponent Relativistic Density Functional Theory Calculations of EPR g- and Hyperfine-Coupling Tensors Using Hybrid Functionals: Validation on Transition-Metal Complexes with Large Tensor Anisotropies and Higher-Order Spin-Orbit Effects. $J$. Phys. Chem. A 2015, 119 (51), 12892-12905. https://doi.org/10.1021/acs.jpca.5b10996.

(4) Malkin, E.; Repiský, M.; Komorovský, S.; Mach, P.; Malkina, O. L.; Malkin, V. G. Effects of Finite Size Nuclei in Relativistic Four-Component Calculations of Hyperfine Structure. J. Chem. Phys. 2011, 134 (4), 044111. https://doi.org/10.1063/1.3526263.

(5) Komorovský, S.; Repiský, M.; Malkina, O. L.; Malkin, V. G.; Malkin Ondík, I.; Kaupp, M. A Fully Relativistic Method for Calculation of Nuclear Magnetic Shielding Tensors with a Restricted Magnetically Balanced Basis in the Framework of the Matrix Dirac-Kohn-Sham Equation. J. Chem. Phys. 2008, 128 (10), 104101. https://doi.org/10.1063/1.2837472.

(6) Komorovský, S.; Repiský, M.; Malkina, O. L.; Malkin, V. G. Fully Relativistic Calculations of NMR Shielding Tensors Using Restricted Magnetically Balanced Basis and Gauge Including Atomic Orbitals. J. Chem. Phys. 2010, 132 (15), 154101. https://doi.org/10.1063/1.3359849.

(7) Kutzelnigg, W.; Fleischer, U.; Schindler, M. The IGLO-Method: Ab-Initio Calculation and Interpretation of NMR Chemical Shifts and Magnetic Susceptibilities. In Deuterium and Shift Calculation; NMR Basic Principles and Progress; Springer, Berlin, Heidelberg, 1990; pp 165-262. https://doi.org/10.1007/978-3-642-75932-1_3.

(8) Dyall, K. Relativistic Double-Zeta, Triple-Zeta, and Quadruple-Zeta Basis Sets for the 4d Elements Y-Cd. Theor. Chem. Acc. Theory Comput. Model. 2007, 117 (4), 483489. https://doi.org/10.1007/s00214-006-0174-5.

(9) Perdew, J. P.; Burke, K.; Ernzerhof, M. Generalized Gradient Approximation Made Simple. Phys. Rev. Lett. 1996, 77 (18), 3865-3868. https://doi.org/10.1103/PhysRevLett.77.3865.

(10) Perdew, J. P.; Ernzerhof, M.; Burke, K. Rationale for Mixing Exact Exchange with Density Functional Approximations. J. Chem. Phys. 1996, 105 (22), 9982-9985. https://doi.org/10.1063/1.472933.

(11) Adamo, C.; Barone, V. Toward Chemical Accuracy in the Computation of NMR Shieldings: The PBE0 Model. Chem. Phys. Lett. 1998, 298 (1), 113-119. https://doi.org/10.1016/S0009-2614(98)01201-9.

(12) Novotný, J.; Přichystal, D.; Sojka, M.; Komorovsky, S.; Nečas, M.; Marek, R. Hyperfine Effects in Ligand NMR: Paramagnetic Ru(III) Complexes with 3-Substituted Pyridines. Inorg. Chem. 2018, 57 (2), 641-652. https://doi.org/10.1021/acs.inorgchem.7b02440.

(13) Komorovsky, S.; Repisky, M.; Ruud, K.; Malkina, O. L.; Malkin, V. G. FourComponent Relativistic Density Functional Theory Calculations of NMR Shielding Tensors for Paramagnetic Systems. J. Phys. Chem. A 2013, 117 (51), 14209-14219. https://doi.org/10.1021/jp408389h. 
(14) Autschbach, J.; Patchkovskii, S.; Pritchard, B. Calculation of Hyperfine Tensors and Paramagnetic NMR Shifts Using the Relativistic Zeroth-Order Regular Approximation and Density Functional Theory. J. Chem. Theory Comput. 2011, 7 (7), 2175-2188. https://doi.org/10.1021/ct200143w.

(15) Martin, B.; Autschbach, J. Temperature Dependence of Contact and Dipolar NMR Chemical Shifts in Paramagnetic Molecules. J. Chem. Phys. 2015, 142 (5), 054108. https://doi.org/10.1063/1.4906318.

(16) Jeremias, L.; Novotný, J.; Repisky, M.; Komorovsky, S.; Marek, R. Interplay of Through-Bond Hyperfine and Substituent Effects on the NMR Chemical Shifts in $\mathrm{Ru}(\mathrm{III})$ Complexes. Inorg. Chem. 2018, 57 (15), 8748-8759. https://doi.org/10.1021/acs.inorgchem.8b00073.

(17) Silvernail, C. M.; Yap, G.; Sommer, R. D.; Rheingold, A. L.; Day, V. W.; Belot, J. A. An Effective Synthesis of Alkyl $\beta$-Cyano- $\alpha, \gamma$-Diketones Using Chlorosulfonylisocyanate and a Representative $\mathrm{Cu}(\mathrm{II})$ Complex. Polyhedron 2001, 20 (26), 3113-3117. https://doi.org/10.1016/S0277-5387(01)00935-4.

(18) Wang, Y.; Wang, L.; Chen, J.; Wang, S.; Yang, Y.; Chen, Q. Synthesis and Evaluation of Ethylene Trimerization Catalysts with Heteroatomic Multidentate Ligands Containing a Phosphorous Atom. React. Kinet. Mech. Catal. 2012, 106 (1), 177-184. https://doi.org/10.1007/s11144-011-0418-9.

(19) Muena, J. P.; Villagrán, M.; Costamagna, J.; Aguirre, M. J. Dinaphthotetraaza[14]Annulene Copper(II) Complexes in the Electrocatalytic Reduction of Carbon Dioxide and Bisulfite Anion. J. Coord. Chem. 2008, 61 (4), 479489. https://doi.org/10.1080/00958970701370559.

(20) Collman, J. P.; Moss, R. A.; Maltz, H.; Heindel, C. C. The Reaction of Metal Chelates. I. Halogenation of Metal Chelates of 1,3-Diketones1,2. J. Am. Chem. Soc. 1961, 83 (3), 531-534. https://doi.org/10.1021/ja01464a006. 


\section{CARTESIAN COORDINATES}

\section{Isolated molecules}

\section{Compound 1a}

\begin{tabular}{|c|c|c|c|}
\hline $\mathrm{Cr}$ & 4.7415188 & 7.4455305 & 1.3629873 \\
\hline 0 & 6.6229587 & 7.9358276 & 1.6336088 \\
\hline 0 & 4.3559757 & 7.8144072 & 3.2517739 \\
\hline O & 5.1115619 & 7.0852690 & -0.5309308 \\
\hline O & 5.1754679 & 5.5863833 & 1.8195300 \\
\hline 0 & 4.3197010 & 9.3102576 & 0.9197680 \\
\hline 0 & 2.8666791 & 6.9367397 & 1.0805140 \\
\hline $\mathrm{C}$ & 7.1744852 & 8.3016853 & 2.7102280 \\
\hline $\mathrm{C}$ & 5.1704884 & 8.1846484 & 4.1441980 \\
\hline $\mathrm{C}$ & 5.4915843 & 5.9955085 & -1.0457383 \\
\hline $\mathrm{C}$ & 5.5374699 & 4.6664193 & 1.0323305 \\
\hline $\mathrm{C}$ & 3.2010683 & 9.7831989 & 0.5704679 \\
\hline $\mathrm{C}$ & 1.9128751 & 7.6848792 & 0.7231859 \\
\hline $\mathrm{C}$ & 6.5306218 & 8.4329291 & 3.9431349 \\
\hline $\mathrm{C}$ & 5.7095038 & 4.8052855 & -0.3472185 \\
\hline $\mathrm{C}$ & 2.0152616 & 9.0528713 & 0.4584703 \\
\hline $\mathrm{C}$ & 8.6386379 & 8.6074458 & 2.6063683 \\
\hline $\mathrm{C}$ & 4.5826535 & 8.3631999 & 5.5118989 \\
\hline $\mathrm{C}$ & 5.7104138 & 6.0312065 & -2.5286829 \\
\hline C & 5.7932522 & 3.3373062 & 1.6772386 \\
\hline $\mathrm{C}$ & 3.1945163 & 11.2516428 & 0.2673102 \\
\hline C & 0.5839338 & 7.0044650 & 0.5853913 \\
\hline $\mathrm{H}$ & 7.1188561 & 8.7501393 & 4.7913880 \\
\hline $\mathrm{H}$ & 6.0290192 & 3.9394858 & -0.9080115 \\
\hline $\mathrm{H}$ & 1.1224681 & 9.5787866 & 0.1542549 \\
\hline $\mathrm{H}$ & 9.0673977 & 8.9201844 & 3.5560899 \\
\hline $\mathrm{H}$ & 8.7857468 & 9.3932790 & 1.8630650 \\
\hline $\mathrm{H}$ & 9.1634831 & 7.7214762 & 2.2437810 \\
\hline $\mathrm{H}$ & 5.3250086 & 8.6628310 & 6.2485640 \\
\hline $\mathrm{H}$ & 4.1153551 & 7.4271703 & 5.8231681 \\
\hline $\mathrm{H}$ & 3.7928057 & 9.1155328 & 5.4652226 \\
\hline $\mathrm{H}$ & 6.0414284 & 5.0732873 & -2.9242039 \\
\hline $\mathrm{H}$ & 6.4524361 & 6.7977742 & -2.7598603 \\
\hline $\mathrm{H}$ & 4.7812179 & 6.3265116 & -3.0199276 \\
\hline $\mathrm{H}$ & 6.0954116 & 2.5772912 & 0.9598657 \\
\hline $\mathrm{H}$ & 4.8878361 & 3.0127828 & 2.1934443 \\
\hline $\mathrm{H}$ & 6.5704191 & 3.4520209 & 2.4353093 \\
\hline $\mathrm{H}$ & 2.2155270 & 11.6080298 & -0.0460453 \\
\hline $\mathrm{H}$ & 3.5139667 & 11.8006545 & 1.1553696 \\
\hline $\mathrm{H}$ & -0.2040950 & 7.6879098 & 0.2763927 \\
\hline $\mathrm{H}$ & 0.3174987 & 6.5486296 & 1.5408680 \\
\hline $\mathrm{H}$ & 0.6707733 & 6.1953428 & -0.1423153 \\
\hline $\mathrm{H}$ & 3.9264202 & 11.4562962 & -0.5162121 \\
\hline
\end{tabular}

\section{Compound 1b}

$\begin{array}{ll}\text { 46 } & \\ \text { Cr } & 4.7514541 \\ \text { O } & 6.6212384 \\ \text { O } & 4.3790773 \\ \text { O } & 5.1302269 \\ \text { O } & 5.1876875 \\ \text { O } & 4.3107568 \\ \text { O } & 2.8792520 \\ \text { C } & 7.1919864 \\ \text { C } & 5.1621140 \\ \text { C } & 5.4939477 \\ \text { C } & 5.5329992 \\ \text { C } & 3.2094920 \\ \text { C } & 1.9090647 \\ \text { C } & 6.5388021 \\ \text { C } & 5.7004985 \\ \text { C } & 2.0073113\end{array}$

7.4452424
7.9457789
7.8136212
7.0708344
5.5880173
9.2991774
6.9506288
8.3202909
8.1879987
5.9966093
4.6490745
9.7961506
7.6695586
8.4512155
4.7853178
9.0564982

1. 3623109

1. 6527890

3. 2480193 $-0.5213710$

1.8001688

0.9164515

1. 0748560

2. 7083970

4. 1573373

$-1.0636577$

1. 0389485

0.5694243

0.7251782

3. 9576341

$-0.3603332$

0.4583564 


$\begin{array}{rr}\mathrm{H} & 6.7925366 \\ \mathrm{H} & 2.8782795 \\ \mathrm{H} & 2.5059916 \\ \mathrm{H} & -0.1590773 \\ \mathrm{H} & 0.7091785 \\ \mathrm{H} & 0.2104397 \\ \mathrm{H} & 4.2122951\end{array}$

2. 9882971

11.4325766

11.7890055

7.4516847

5.9373989

7.0646854

11.6443447
1.5115016

$-0.7749113$

0.8978649

1.2399580

0.8644719

$-0.4265865$

0.3921490

\section{Compound 1d}

43

Cr $\quad 4.7508352$

o $\quad 6.6145269$

o $\quad 4.3923994$

○ $\quad 5.1326555$

5.1848901

4.3010960

2.8788783

7. 1945404

5.1772745

5.4968776

5.5297111

3. 1985906

1.9020175

6.5418326

5.6955598

2.0103598

8.6500922

4.5615681

5.7088022

5.7616066

3.2238801

0.5907141

Br 7.5743979

Br 6.2295431

Br 0.4391813

$\mathrm{H} \quad 8.8503191$

$\mathrm{H} \quad 8.9443258$

$\mathrm{H} \quad 9.2465717$

$\mathrm{H} \quad 5.0611005$

$\mathrm{H} \quad 3.5069798$

$\mathrm{H} \quad 4.6776653$

$\mathrm{H} \quad 6.7297051$

$\mathrm{H} \quad 5.5120742$

$\mathrm{H} \quad 5.0481103$

$\mathrm{H} \quad 5.1054507$

$\mathrm{H} \quad 5.5732584$

$\mathrm{H} \quad 6.7849146$

$\mathrm{H} \quad 2.9057822$

$\mathrm{H} \quad 2.5269817$

$\mathrm{H} \quad-0.1577930$

$\mathrm{H} \quad 0.7296150$

H $\quad 0.2084068$

$\mathrm{H} \quad 4.2343103$

\section{Compound 1e}

43

Cr 4.7508023

o 6.6100995

o 4.4013921

o $\quad 5.1356502$

o $\quad 5.1846657$

o 4.2936168

O $\quad 2.8788496$

C $\quad 7.1951737$

C $\quad 5.1812147$

C $\quad 5.5007837$

C $\quad 5.5302907$

C $\quad 3.1934957$

C $\quad 1.8978835$
7.4474404

7.9540872

7. 8111560

7.0675346

5.5904636

9.2945192

6.9631222

8.3290069

8.1839289

5. 9905846

4. 6438780

9.7957265

7.6788483

8.4478356

4. 7902447

9.0527591

8.6464950

8.3373507

6.0615046

3. 3262771

11.2598349

6.9687686

9.0099676

3.2599141

9.9716588

9.6751341

8.5001804

8.0059502

7.6912942

8.0811427

9.3604139

5.7737869

7.0769131

5. 3629332

2.5615886

3. 4356867

2. 9850739

11.4413422

11.8005966

7. 4283430

5. 9254937

7. 0413607

11.6323811
1.3679553

1. 6637890

3. 2535717

$-0.5102866$

1. 7928696

0.9180980

1. 0854365

2. 7218517

4. 1707752

$-1.0616264$

1. 0303410

0.5580700

0.7249339

3. 9609687

$-0.3578014$

0.4487622

2. 5832321

5. 5257167

$-2.5410846$

1. 7001156

0.2505481

0.6032024

5. 4549911

$-1.3515409$

$-0.1003771$

2. 8877911

1. 5470095

3. 2353500

6.2496730

5.4633337

5.8876462

$-2.7978722$

$-2.8757199$

$-3.0576173$

1. 2808924

2. 7651219

1.5339958

$-0.7775882$

0.8932335

1. 2508062

0.8752208

$-0.4165858$

0.3988388
7. 4464920

7. 9549333

7. 8113631

7.0585976

5. 5899104

9. 2888825

6.9722625

8.3317271

8.1851672

5. 9845202

4.6392827

9.7965162

7. 6837202
1.3669774

1. 6713144

3. 2515693

$-0.5064893$

1. 7825452

0.9158364

1.0839315

2. 7261896

4. 1729640

$-1.0633457$

1.0250311

0.5567066

0.7253173 


$\begin{array}{lr}\mathrm{C} & 6.5492005 \\ \mathrm{C} & 5.7009731 \\ \mathrm{C} & 1.9974430 \\ \mathrm{C} & 8.6502539 \\ \mathrm{C} & 4.5424550 \\ \mathrm{C} & 5.7089437 \\ \mathrm{C} & 5.7548061 \\ \mathrm{C} & 3.2441313 \\ \mathrm{C} & 0.5963098 \\ \mathrm{I} & 7.6882559 \\ \mathrm{I} & 6.2911967 \\ \mathrm{I} & 0.2629211 \\ \mathrm{H} & 8.8602492 \\ \mathrm{H} & 8.9260293 \\ \mathrm{H} & 9.2571728 \\ \mathrm{H} & 5.0306429 \\ \mathrm{H} & 3.4904220 \\ \mathrm{H} & 4.6442581 \\ \mathrm{H} & 6.7306563 \\ \mathrm{H} & 5.5067137 \\ \mathrm{H} & 5.0492118 \\ \mathrm{H} & 5.0973847 \\ \mathrm{H} & 5.5615830 \\ \mathrm{H} & 6.7780072 \\ \mathrm{H} & 2.9343140 \\ \mathrm{H} & 2.5568145 \\ \mathrm{H} & -0.1571653 \\ \mathrm{H} & 0.7546856 \\ \mathrm{H} & 0.2069896 \\ \mathrm{H} & 4.2608319 \\ & \end{array}$

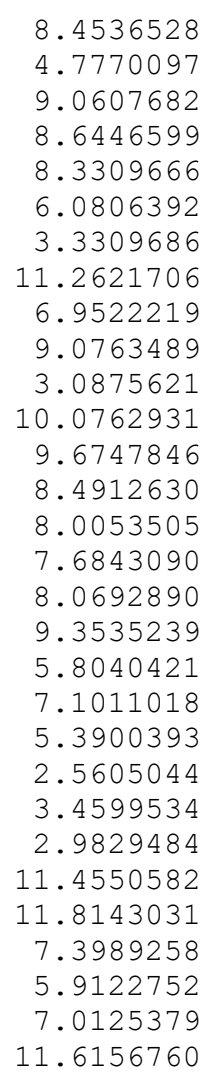

\section{Compound 2a}

$\begin{array}{lrrr}29 & & & \\ \mathrm{Cu} & -0.0001166 & 0.0001583 & -0.0001206 \\ \mathrm{C} & 3.2713981 & 2.5001741 & -0.8602257 \\ \mathrm{C} & 2.1219984 & 1.9148579 & -0.0937551 \\ \mathrm{O} & 1.5087291 & 0.9765806 & -0.6783300 \\ \mathrm{C} & 1.8099717 & 2.4217987 & 1.1699638 \\ \mathrm{C} & 0.7600913 & 1.9421201 & 1.9567161 \\ \mathrm{O} & -0.0276252 & 1.0068147 & 1.6357783 \\ \mathrm{C} & 0.5069275 & 2.5553581 & 3.3025507 \\ \mathrm{H} & 3.9892913 & 1.7093933 & -1.0857781 \\ \mathrm{H} & 3.7700783 & 3.3002085 & -0.3170026 \\ \mathrm{H} & 2.9055958 & 2.8810891 & -1.8156039 \\ \mathrm{H} & 2.4130850 & 3.2283746 & 1.5597872 \\ \mathrm{H} & -0.5135187 & 2.9419120 & 3.3320708 \\ \mathrm{H} & 1.2061785 & 3.3563230 & 3.5336594 \\ \mathrm{H} & 0.5763628 & 1.7790091 & 4.0668649 \\ \mathrm{O} & -1.5072233 & -0.9781369 & 0.6792904 \\ \mathrm{C} & -2.1202084 & -1.9167438 & 0.0949508 \\ \mathrm{C} & -3.2671108 & -2.5047017 & 0.8631373 \\ \mathrm{H} & -3.9843633 & -1.7148185 & 1.0937236 \\ \mathrm{H} & -3.7673253 & -3.3031221 & 0.3189502 \\ \mathrm{H} & -2.8980941 & -2.8887901 & 1.8160273 \\ \mathrm{C} & -1.8099340 & -2.4218468 & -1.1699348 \\ \mathrm{C} & -0.7620165 & -1.9400825 & -1.9580301 \\ \mathrm{O} & 0.0256601 & -1.0047180 & -1.6371607 \\ \mathrm{C} & -0.5111551 & -2.5509058 & -3.3053936 \\ \mathrm{H} & 0.5103997 & -2.9341590 & -3.3387115 \\ \mathrm{H} & -1.2087180 & -3.3536719 & -3.5353529 \\ \mathrm{H} & -0.5855420 & -1.7737941 & -4.0684908 \\ \mathrm{H} & -2.4128162 & -3.2286809 & -1.5595803\end{array}$

\section{Compound 2b}

$\begin{array}{lrrr}31 & & & \\ \mathrm{Cu} & -0.0010788 & 0.0012377 & -0.0008483 \\ \mathrm{C} & 3.3364938 & 2.4416129 & -0.8248386 \\ \mathrm{C} & 2.1606847 & 1.8836481 & -0.0869644 \\ \mathrm{O} & 1.5377279 & 0.9456863 & -0.6445206\end{array}$




$$
\begin{array}{r}
1.8170136 \\
0.7243121 \\
-0.0495624 \\
0.4507192 \\
3.4647361 \\
4.2400609 \\
3.1899633 \\
-0.4180456 \\
0.2787143 \\
1.3179824 \\
-1.5427427 \\
-2.1649667 \\
-3.3441383 \\
-3.4697261 \\
-4.2468493 \\
-3.2048923 \\
-1.8177768 \\
-0.7225752 \\
0.0503883 \\
-0.4450332 \\
0.4234868 \\
-0.2703885 \\
-1.3113025 \\
-2.6092110 \\
2.6093996 \\
-3.2534320 \\
3.2543833
\end{array}
$$

2.4289494

1.9735777

1.0456795

2. 6209636

1. 9058501

2. 3583074

3.5059688

2.1565583

3. 6911640

2.5235477

$-0.9400489$

$-1.8788155$

$-2.4330660$

$-1.9002442$

$-2.3409080$

$-3.4991998$

$-2.4280127$

$-1.9754141$

$-1.0465108$

$-2.6271621$

$-2.1624644$

$-3.6963732$

$-2.5347862$

$-3.4880256$

3.4879063

$-4.3509811$

4. 3500114
1.1736838

1.9504199

1. 6055758

3.2711900

$-1.7615711$

$-0.2177821$

$-1.0195809$

3.7300144

3. 1403600

3.9273356

0.6406382

0.0836172

0.8189281

1.7576971

0.2117518

1.0088567

$-1.1743732$

$-1.9492003$

$-1.6050212$

$-3.2669977$

$-3.7259874$

$-3.1314555$

$-3.9251374$

$-1.6855419$

1. 6855672

$-2.1015991$

2.1022050

\section{Compound 2c}

\section{9}

$\mathrm{Cu} \quad 0.0011297$

C $\quad 3.3230153$

C $\quad 2.1558278$

O $\quad 1.5284716$

C $\quad 1.8127691$

C $\quad 0.7368639$

O $\quad-0.0393856$

C $\quad 0.4516122$

$\mathrm{H} \quad 3.4204555$

$\mathrm{H} \quad 4.2396398$

$\mathrm{H} \quad 3.1858517$

Cl $\quad 2.7848779$

$\mathrm{H} \quad-0.4145556$

$\begin{array}{ll}\mathrm{H} & 0.2637855 \\ \mathrm{H} & 1.3136368\end{array}$

O $\quad-1.5417231$

C $\quad-2.1690535$

C $\quad-3.3513789$

$\mathrm{H} \quad-3.4553939$

$\mathrm{H} \quad-4.2593084$

$\mathrm{H} \quad-3.2280537$

C $\quad-1.8132307$

C $\quad-0.7224007$

-0.7224007
0.0571281

$-0.4236984$

0.4525989

$-0.2462806$

$-1.2754672$

$-2.7877337$

\section{Compound 2d}

29

$\mathrm{Cu} \quad 0.0024555$

C $\quad 3.3187950$

C $\quad 2.1565099$

O 1.5263993

C $\quad 1.8148436$

C $\quad 0.7385033$

o $\quad-0.0356432$
-0.0012735
2.4479501
1.8964749
0.9534936
2.4249369
1.9739532
1.0390252
2.6043949
1.9076646
2.3525944
3.5133868
3.7258335
2.1182837
3.6733487
2.5090396
-0.9388935
-1.8818374
-2.4165734
-1.8691642
-2.3152733
-3.4821180
-2.4243942
-1.9899887
-1.0587782
-2.6352686
-2.1604215
-3.7045623
-2.5392886
-3.7225446

0.0009434

$-0.8418490$

$-0.0839379$

$-0.6440622$

1.1709226

1.9523539

1. 6059710

3.2798247

$-1.7800324$

$-0.2570722$

$-1.0350506$

1. 7988190

3.7214606

3.1644625

3.9425300

0.6341758

0.0739557

0.8203229

1.7536569

0.2230556

1. 0218849

$-1.1712997$

$-1.9413359$

$-1.5923257$

$-3.2586746$

$-3.6925108$

$-3.1305975$

$-3.9344054$

$-1.8011854$

$$
\begin{array}{rr}
-0.0028182 & 0.0020240 \\
2.4403483 & -0.8578653 \\
1.8990585 & -0.0844350 \\
0.9552040 & -0.6404305 \\
2.4273127 & 1.1722979 \\
1.9746456 & 1.9544382 \\
1.0393737 & 1.6036076
\end{array}
$$




$\begin{array}{lrrr}\mathrm{C} & 0.4393146 & 2.5927641 & 3.2850773 \\ \mathrm{H} & 3.4022618 & 1.8920792 & -1.7928575 \\ \mathrm{H} & 4.2417523 & 2.3441952 & -0.2833871 \\ \mathrm{H} & 3.1852924 & 3.5048578 & -1.0582708 \\ \mathrm{Br} & 2.8768328 & 3.8473773 & 1.8582440 \\ \mathrm{H} & -0.4278620 & 2.0968380 & 3.7138194 \\ \mathrm{H} & 0.2452291 & 3.6614275 & 3.1782521 \\ \mathrm{H} & 1.2954202 & 2.4964459 & 3.9551630 \\ \mathrm{O} & -1.5382582 & -0.9423100 & 0.6317332 \\ \mathrm{C} & -2.1708273 & -1.8832853 & 0.0736504 \\ \mathrm{C} & -3.3517355 & -2.4039915 & 0.8328725 \\ \mathrm{H} & -3.4414429 & -1.8490561 & 1.7633407 \\ \mathrm{H} & -4.2645504 & -2.2980715 & 0.2440348 \\ \mathrm{H} & -3.2364723 & -3.4690832 & 1.0411887 \\ \mathrm{C} & -1.8159017 & -2.4260730 & -1.1731762 \\ \mathrm{C} & -0.7215537 & -1.9934110 & -1.9415129 \\ \mathrm{O} & 0.0568568 & -1.0630119 & -1.5871949 \\ \mathrm{C} & -0.4070972 & -2.6283052 & -3.2606742 \\ \mathrm{H} & 0.4740397 & -2.1479824 & -3.6785813 \\ \mathrm{H} & -0.2294803 & -3.6984026 & -3.1405557 \\ \mathrm{H} & -1.2498443 & -2.5268460 & -3.9467697 \\ \mathrm{Br} & -2.8838376 & -3.8392803 & -1.8640327\end{array}$

\section{Compound 2e}

$\begin{array}{lrrr}29 & & & \\ \mathrm{Cu} & 0.0001509 & -0.0001804 & 0.0001604 \\ \mathrm{C} & 3.3228086 & 2.4207441 & -0.8690200 \\ \mathrm{C} & 2.1602271 & 1.8994267 & -0.0801453 \\ \mathrm{O} & 1.5259321 & 0.9561226 & -0.6329406 \\ \mathrm{C} & 1.8220418 & 2.4370678 & 1.1769527 \\ \mathrm{C} & 0.7389927 & 1.9801427 & 1.9527845 \\ \mathrm{O} & -0.0328888 & 1.0444050 & 1.5972579 \\ \mathrm{C} & 0.4187539 & 2.5855150 & 3.2857218 \\ \mathrm{H} & 3.3923397 & 1.8596106 & -1.7976600 \\ \mathrm{H} & 4.2507259 & 2.3211213 & -0.3030218 \\ \mathrm{H} & 3.1993950 & 3.4836625 & -1.0842347 \\ \mathrm{I} & 2.9952688 & 4.0043146 & 1.9349783 \\ \mathrm{H} & -0.4502738 & 2.0786339 & 3.6979482 \\ \mathrm{H} & 0.2155746 & 3.6534047 & 3.1874674 \\ \mathrm{H} & 1.2650339 & 2.4888755 & 3.9682553 \\ \mathrm{O} & -1.5375667 & -0.9432412 & 0.6241963 \\ \mathrm{C} & -2.1710104 & -1.8874598 & 0.0719881 \\ \mathrm{C} & -3.3447047 & -2.3964414 & 0.8524181 \\ \mathrm{H} & -3.4200067 & -1.8289385 & 1.7767260 \\ \mathrm{H} & -4.2662644 & -2.2945471 & 0.2765123 \\ \mathrm{H} & -3.2302307 & -3.4587258 & 1.0755118 \\ \mathrm{C} & -1.8227599 & -2.4362731 & -1.1775152 \\ \mathrm{C} & -0.7283635 & -1.9919685 & -1.9446976 \\ \mathrm{O} & 0.0456171 & -1.0586114 & -1.5874855 \\ \mathrm{C} & -0.3965120 & -2.6101907 & -3.2688707 \\ \mathrm{H} & 0.4802438 & -2.1118997 & -3.6751676 \\ \mathrm{H} & -0.2008594 & -3.6784045 & -3.1594710 \\ \mathrm{H} & -1.2342794 & -2.5142578 & -3.9619190 \\ \mathrm{I} & -2.9973855 & -4.0019068 & -1.9367303\end{array}$

\section{Compound $\mathrm{Cr}(\mathrm{Me}-\mathrm{acac})_{3}$}

$\begin{array}{lllr}52 & & & \\ \mathrm{Cr} & 4.7913635 & 7.5932676 & 1.4493183 \\ \mathrm{O} & 6.6296831 & 8.1997461 & 1.7212704 \\ \mathrm{O} & 4.4587395 & 7.9428197 & 3.3423256 \\ \mathrm{O} & 5.1594759 & 7.2111861 & -0.4334982 \\ \mathrm{O} & 5.3267887 & 5.7602153 & 1.8699595 \\ \mathrm{O} & 4.2291791 & 9.4075171 & 0.9862891 \\ \mathrm{O} & 2.9403796 & 7.0167478 & 1.1963604 \\ \mathrm{C} & 7.2383132 & 8.4268267 & 2.8068469 \\ \mathrm{C} & 5.2920732 & 8.1820703 & 4.2639093 \\ \mathrm{C} & 5.4051480 & 6.0991510 & -0.9845318 \\ \mathrm{C} & 5.5449132 & 4.7961445 & 1.0802618 \\ \mathrm{C} & 3.1357180 & 9.7946821 & 0.4822681\end{array}$




\begin{tabular}{|c|c|c|c|}
\hline $\mathrm{C}$ & 1.9752758 & 7.6526350 & 0.6804978 \\
\hline $\mathrm{C}$ & 6.6727103 & 8.3805362 & 4.0926772 \\
\hline $\mathrm{C}$ & 5.5540080 & 4.8675229 & -0.3236279 \\
\hline $\mathrm{C}$ & 2.0052584 & 8.9904696 & 0.2530460 \\
\hline $\mathrm{C}$ & 8.6898510 & 8.7851249 & 2.6446341 \\
\hline $\mathrm{C}$ & 4.6910981 & 8.2677404 & 5.639890 \\
\hline $\mathrm{C}$ & 5.5490093 & 6.1649057 & -2.4802562 \\
\hline $\mathrm{C}$ & 5.8290903 & 3.4858051 & 1.7613800 \\
\hline $\mathrm{C}$ & 3.0939364 & 11.2571656 & 0.1335645 \\
\hline $\mathrm{C}$ & 0.7060795 & 6.8573212 & 0.547168 \\
\hline $\mathrm{H}$ & 8.9555998 & 9.6690208 & 3.2259247 \\
\hline $\mathrm{H}$ & 8.8947818 & 8.9594319 & 1.5911006 \\
\hline $\mathrm{H}$ & 9.3231788 & 7.9652667 & $2.9941^{\circ}$ \\
\hline $\mathrm{H}$ & 4.9976821 & 7.4073996 & 6.240601 \\
\hline $\mathrm{H}$ & 3.6073309 & 8.2633912 & 5.5 \\
\hline $\mathrm{H}$ & 5.0171968 & 9.1645205 & 6.16 \\
\hline $\mathrm{H}$ & 6.4409059 & 5.6401812 & -2.8250 \\
\hline $\mathrm{H}$ & 5.5928189 & 7.2079115 & -2.784428 \\
\hline $\mathrm{H}$ & 4.6888146 & 5.6942935 & -2.9 \\
\hline $\mathrm{H}$ & 4.9967438 & 2.7931146 & 1.6 \\
\hline $\mathrm{H}$ & 5.9494416 & 3.6609794 & 2.8 \\
\hline $\mathrm{H}$ & 6.7238056 & 3.0076599 & 1.36042 \\
\hline $\mathrm{H}$ & 3.0871707 & 11.3875258 & -0.9517 \\
\hline $\mathrm{H}$ & 2.1968999 & 11.7401559 & 0.5233 \\
\hline $\mathrm{H}$ & -0.1598688 & 7.4117336 & 0.91088 \\
\hline $\mathrm{H}$ & 0.8113620 & 5.9286336 & 1.10284 \\
\hline $\mathrm{H}$ & 0.5193228 & 6.6179558 & -0.5032 \\
\hline $\mathrm{H}$ & 3.9799727 & 11.7411247 & 0.5 \\
\hline $\mathrm{C}$ & 5.8026912 & 3.6194280 & -1.128518 \\
\hline $\mathrm{H}$ & 5.3084655 & 3.6635611 & -2.098829 \\
\hline $\mathrm{H}$ & 6.8665520 & 3.4362800 & -1.3194564 \\
\hline $\mathrm{H}$ & 5.4128 & 2.7347186 & -0.6 \\
\hline $\mathrm{C}$ & 0.7807969 & 9.5901202 & -0.3855 \\
\hline $\mathrm{H}$ & 0.2365569 & 8.8538204 & -0.97716 \\
\hline $\mathrm{H}$ & 0.0727667 & 10.0006818 & 0.343737 \\
\hline $\mathrm{H}$ & 1.0409977 & 10.3994728 & -1.06684 \\
\hline $\mathrm{C}$ & 7.5442818 & 8.6288521 & 5.294967 \\
\hline $\mathrm{H}$ & 7.6288689 & 9.6910318 & 5.552852 \\
\hline $\mathrm{H}$ & 7.1632628 & 8.1164549 & 6.17 \\
\hline $\mathrm{H}$ & 8.5571274 & 8.2579599 & 5.13804 \\
\hline
\end{tabular}

\section{Compound $\mathrm{Cr}\left(\mathrm{NO}_{2} \text {-acac }\right)_{3}$}

1.0558790

0.5688426

0.7741737

3. 9096967

$-0.3463840$

0.5074341

2. 4859629

5. 4760101

$-2.5420085$

1. 7722738

0.2935158

0.6593326

2.3127564

1.6122777

3. 3591775

6.1355856 


$\begin{array}{rrr}3.5143438 & 8.4831295 & 5.3195029 \\ 4.9299787 & 9.3611185 & 5.9735812 \\ 6.5458329 & 5.3971764 & -2.8869604 \\ 5.9072841 & 7.0660761 & -2.7813609 \\ 4.8288394 & 5.6837645 & -3.0670697 \\ 5.3086612 & 2.4497933 & 1.2109919 \\ 5.2139604 & 3.3784994 & 2.7380970 \\ 6.7689320 & 3.0965563 & 1.9240574 \\ 3.3636736 & 11.4295374 & -0.7843727 \\ 2.4803648 & 11.8039246 & 0.6783179 \\ 0.0662815 & 7.0948285 & 1.5686478 \\ 0.8755875 & 5.8858600 & 0.5488068 \\ 0.0383054 & 7.2950832 & -0.1694384 \\ 4.2619436 & 11.6416934 & 0.7344476 \\ 0.8595804 & 9.7824966 & 0.1429104 \\ 6.0256593 & 3.5315056 & -1.0980922 \\ 7.3824628 & 9.0692506 & 5.0148901 \\ 7.2568637 & 8.5402712 & 6.1026369 \\ 8.1217395 & 10.0098449 & 4.7960094 \\ -0.1506327 & 9.5631828 & 0.7836280 \\ 0.9213338 & 10.5676117 & -0.7837076 \\ 5.4380237 & 3.3252284 & -2.1429201 \\ 6.8883904 & 2.8025648 & -0.6469600\end{array}$

\section{Compound $\mathrm{Cu}(\mathrm{Me}-\mathrm{acac})_{2}$}

\section{5}

$\mathrm{Cu} \quad-0.0018994$

C $\quad 3.2790679$

C 2.1296112

o 1.4965289

C $\quad 1.8432770$

1.8432770
0.7259356

$-0.0702136$

0.3888941

3. 2459507

4. 2312971

3. 2435784

$-0.5814854$

0.3715671

1.1369236

$-1.5061903$

$-2.1379393$

$-3.2934172$

$-3.2659760$

$-4.2421786$

$-3.2602078$

$-1.8451983$

$-0.7230811$

0.0722753

$-0.3790297$

0.5938271

$-0.3635785$

$-1.1220646$

$-2.7119138$

$-2.7317658$

$-2.3775990$

$-3.7462077$

2.7107643

3. 7418403

2.3684117

2.7425635
0.0019192

2. 4744132

1. 9200710

0.9888333

2. 4503962

2. 0054422

1.0843257

2. 6505002

2. 0542071

2. 2044106

3. 5635291

2. 2882016

3. 7385999

2.3923976

$-0.9789778$

$-1.9112020$

$-2.4596782$

$-2.0348531$

$-2.1902954$

$-3.5486058$

$-2.4479636$

$-2.0081635$

$-1.0864439$

$-2.6598065$

$-2.3002940$

$-3.7475501$

$-2.4040748$

$-3.5429729$

$-3.5184019$

$-4.5453158$

$-3.4444927$

3. 5445332

3. 4541398

4.5478039

3.5102346
$-0.0009990$

$-0.8767282$

$-0.0787163$

$-0.6570450$

1. 1909637

1. 9182327

1. 5715237

3. 2356982

$-1.8789936$

$-0.4128936$

$-0.9300560$

3. 5663016

3. 1580370

3. 9897716

0.6506474

0.0724689

0.8657651

1. 8662501

0.3945185

0.9243687

$-1.1930646$

$-1.9160518$

$-1.5690902$

$-3.2284933$

$-3.5548304$

$-3.1457790$

$-3.9882911$

$-1.7560594$

$-2.8451979$

$-1.4645212$

$-1.4275450$

1. 7544685

1.4139230

1. 4757206

2.8431181

\section{Compound $\mathrm{Cu}\left(\mathrm{NO}_{2} \text {-acac }\right)_{2}$}

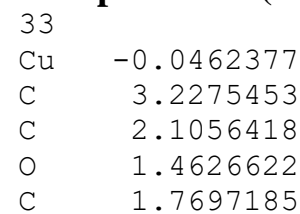

0.0503589

2. 5113640

1. 9602545

1.0252793

2. 4810458
$-0.0312659$

$-0.9558799$

$-0.1315748$

$-0.6758072$

1.1396325 


$$
\begin{array}{rrr}
0.6967770 & 2.0169656 & 1.9355913 \\
-0.0612794 & 1.0787721 & 1.5764334 \\
0.4032628 & 2.5743189 & 3.2939335 \\
3.0552063 & 2.2158702 & -1.9883791 \\
4.1726805 & 2.0885006 & -0.6126666 \\
3.3182489 & 3.5928964 & -0.8751620 \\
-0.1953333 & 1.8412133 & 3.8299711 \\
-0.1652820 & 3.4996601 & 3.1922717 \\
1.3046311 & 2.8180691 & 3.8530238 \\
-1.5939105 & -0.8818699 & 0.5841570 \\
-2.2296650 & -1.8246084 & 0.0447942 \\
-3.4292854 & -2.2899751 & 0.8102521 \\
-3.6966221 & -1.5059626 & 1.5151306 \\
-4.2722807 & -2.5319582 & 0.1658548 \\
-3.1801509 & -3.1991726 & 1.3589574 \\
-1.8671347 & -2.3741832 & -1.2067593 \\
-0.7632683 & -1.9443204 & -1.9791261 \\
0.0076425 & -1.0205934 & -1.6100461 \\
-0.3931914 & -2.5867784 & -3.2799822 \\
0.6493984 & -2.3504246 & -3.4805804 \\
-0.5501158 & -3.6637260 & -3.2781045 \\
-1.0153583 & -2.1780385 & -4.0773189 \\
-2.6805080 & -3.4504807 & -1.7329912 \\
2.5788020 & 3.5627531 & 1.6612343 \\
-3.0192668 & -4.3356429 & -0.9706394 \\
-2.9831028 & -3.4135701 & -2.9105134 \\
3.7869772 & 3.4769844 & 1.5497923 \\
2.0085108 & 4.5014789 & 2.1838088
\end{array}
$$

\section{Supramolecular clusters}

\section{Compound 1a $-\mathrm{X}$-ray cluster}

$\begin{array}{lrrr}387 & & & \\ \mathrm{Cr} & 2.119915 & 2.004090 & 7.603111 \\ \mathrm{O} & 2.262205 & 3.795437 & 8.397835 \\ \mathrm{O} & 3.779460 & 1.453748 & 8.492187 \\ \mathrm{O} & 3.187444 & 2.595170 & 6.068271 \\ \mathrm{O} & 1.943357 & 0.208217 & 6.845223 \\ \mathrm{O} & 0.519482 & 2.545379 & 6.646825 \\ \mathrm{O} & 1.065535 & 1.423572 & 9.143282 \\ \mathrm{C} & 3.060843 & 5.683725 & 9.530546 \\ \mathrm{H} & 2.862516 & 6.248024 & 8.779283 \\ \mathrm{H} & 3.871947 & 5.980962 & 9.948992 \\ \mathrm{H} & 2.340116 & 5.727481 & 10.163870 \\ \mathrm{C} & 3.231106 & 4.257136 & 9.058139 \\ \mathrm{C} & 4.385392 & 3.546481 & 9.369307 \\ \mathrm{H} & 5.070433 & 4.008180 & 9.797124 \\ \mathrm{C} & 4.594583 & 2.205895 & 9.091421 \\ \mathrm{C} & 5.867332 & 1.540505 & 9.556396 \\ \mathrm{H} & 5.673884 & 0.961118 & 10.297966 \\ \mathrm{H} & 6.496934 & 2.210421 & 9.831052 \\ \mathrm{H} & 6.240546 & 1.025998 & 8.837445 \\ \mathrm{C} & 4.251618 & 2.635909 & 3.984767 \\ \mathrm{H} & 4.281838 & 3.572886 & 4.190920 \\ \mathrm{H} & 3.843847 & 2.510676 & 3.124610 \\ \mathrm{H} & 5.144241 & 2.282090 & 3.969580 \\ \mathrm{C} & 3.444604 & 1.917710 & 5.031527 \\ \mathrm{C} & 3.059312 & 0.600510 & 4.819881 \\ \mathrm{H} & 3.284502 & 0.218024 & 4.001892 \\ \mathrm{C} & 2.370109 & -0.192375 & 5.718974 \\ \mathrm{C} & 2.070047 & -1.627262 & 5.399081 \\ \mathrm{H} & 2.374237 & -2.186280 & 6.118355 \\ \mathrm{H} & 2.520833 & -1.875463 & 4.588363 \\ \mathrm{H} & 1.123578 & -1.738915 & 5.286311 \\ \mathrm{C} & -1.711709 & 2.829792 & 6.005262 \\ \mathrm{H} & -1.357341 & 3.547236 & 5.475338 \\ \mathrm{H} & -2.495311 & 3.131556 & 6.470561 \\ \mathrm{H} & -1.942738 & 2.093488 & 5.433332 \\ \mathrm{C} & -0.662859 & 2.377146 & 7.015025\end{array}$




\begin{tabular}{|c|c|c|c|}
\hline $\mathrm{C}$ & -1.059406 & 1.814356 & 8.215431 \\
\hline $\mathrm{H}$ & -1.971070 & 1.702703 & 8.359221 \\
\hline $\mathrm{C}$ & -0.190630 & 1.403957 & 9.222124 \\
\hline C & -0.712787 & 0.878888 & 10.543540 \\
\hline $\mathrm{H}$ & -0.055111 & 0.303273 & 10.940983 \\
\hline $\mathrm{H}$ & -1.521985 & 0.384749 & 10.394903 \\
\hline $\mathrm{H}$ & -0.891896 & 1.615192 & 11.133242 \\
\hline $\mathrm{Cr}$ & 0.835988 & 1.767960 & 15.681214 \\
\hline 0 & 0.978278 & -0.023387 & 16.475938 \\
\hline 0 & 2.495533 & 2.318302 & 16.570290 \\
\hline 0 & 1.903518 & 1.176880 & 14.146374 \\
\hline 0 & 0.659430 & 3.563833 & 14.923326 \\
\hline 0 & -0.764445 & 1.226671 & 14.724928 \\
\hline 0 & -0.218392 & 2.348478 & 17.221385 \\
\hline C & 1.776916 & -1.911675 & 17.608650 \\
\hline $\mathrm{H}$ & 1.578589 & -2.475974 & 16.857386 \\
\hline $\mathrm{H}$ & 2.588021 & -2.208912 & 18.027095 \\
\hline $\mathrm{H}$ & 1.056189 & -1.955431 & 18.241973 \\
\hline $\mathrm{C}$ & 1.947180 & -0.485086 & 17.136242 \\
\hline $\mathrm{C}$ & 3.101465 & 0.225569 & 17.447411 \\
\hline $\mathrm{H}$ & 3.786507 & -0.236130 & 17.875227 \\
\hline C & 3.310657 & 1.566155 & 17.169524 \\
\hline $\mathrm{C}$ & 4.583405 & 2.231545 & 17.634499 \\
\hline $\mathrm{H}$ & 4.389957 & 2.810932 & 18.376069 \\
\hline $\mathrm{H}$ & 5.213007 & 1.561629 & 17.909155 \\
\hline $\mathrm{H}$ & 4.956619 & 2.746052 & 16.915548 \\
\hline $\mathrm{C}$ & 2.967691 & 1.136141 & 12.062870 \\
\hline $\mathrm{H}$ & 2.997912 & 0.199164 & 12.269023 \\
\hline $\mathrm{H}$ & 2.559920 & 1.261374 & 11.202714 \\
\hline $\mathrm{H}$ & 3.860314 & 1.489960 & 12.047683 \\
\hline $\mathrm{C}$ & 2.160677 & 1.854340 & 13.109631 \\
\hline $\mathrm{C}$ & 1.775386 & 3.171540 & 12.897984 \\
\hline $\mathrm{H}$ & 2.000576 & 3.554026 & 12.079996 \\
\hline C & 1.086182 & 3.964425 & 13.797077 \\
\hline C & 0.786121 & 5.399312 & 13.477184 \\
\hline $\mathrm{H}$ & 1.090310 & 5.958330 & 14.196459 \\
\hline $\mathrm{H}$ & 1.236907 & 5.647513 & 12.666466 \\
\hline $\mathrm{H}$ & -0.160349 & 5.510965 & 13.364414 \\
\hline $\mathrm{C}$ & -2.995635 & 0.942258 & 14.083365 \\
\hline $\mathrm{H}$ & -2.641268 & 0.224814 & 13.553442 \\
\hline $\mathrm{H}$ & -3.779237 & 0.640494 & 14.548664 \\
\hline $\mathrm{H}$ & -3.226664 & 1.678562 & 13.511436 \\
\hline $\mathrm{C}$ & -1.946785 & 1.394904 & 15.093128 \\
\hline $\mathrm{C}$ & -2.343333 & 1.957694 & 16.293534 \\
\hline $\mathrm{H}$ & -3.254996 & 2.069347 & 16.437325 \\
\hline $\mathrm{C}$ & -1.474556 & 2.368093 & 17.300228 \\
\hline $\mathrm{C}$ & -1.996714 & 2.893162 & 18.621644 \\
\hline $\mathrm{H}$ & -1.339038 & 3.468777 & 19.019086 \\
\hline $\mathrm{H}$ & -2.805911 & 3.387301 & 18.473007 \\
\hline $\mathrm{H}$ & -2.175822 & 2.156858 & 19.211345 \\
\hline $\mathrm{Cr}$ & 3.403842 & 1.767960 & -0.474992 \\
\hline 0 & 3.546131 & -0.023387 & 0.319731 \\
\hline 0 & 5.063387 & 2.318302 & 0.414084 \\
\hline 0 & 4.471371 & 1.176880 & -2.009832 \\
\hline 0 & 3.227283 & 3.563833 & -1.232880 \\
\hline 0 & 1.803408 & 1.226671 & -1.431278 \\
\hline 0 & 2.349462 & 2.348478 & 1.065179 \\
\hline $\mathrm{C}$ & 4.344769 & -1.911675 & 1.452443 \\
\hline $\mathrm{H}$ & 4.146443 & -2.475974 & 0.701179 \\
\hline $\mathrm{H}$ & 5.155874 & -2.208912 & 1.870889 \\
\hline $\mathrm{H}$ & 3.624042 & -1.955431 & 2.085766 \\
\hline $\mathrm{C}$ & 4.515033 & -0.485086 & 0.980035 \\
\hline $\mathrm{C}$ & 5.669318 & 0.225569 & 1.291204 \\
\hline $\mathrm{H}$ & 6.354360 & -0.236130 & 1.719020 \\
\hline $\mathrm{C}$ & 5.878510 & 1.566155 & 1.013317 \\
\hline $\mathrm{C}$ & 7.151258 & 2.231545 & 1.478293 \\
\hline $\mathrm{H}$ & 6.957810 & 2.810932 & 2.219863 \\
\hline $\mathrm{H}$ & 7.780861 & 1.561629 & 1.752948 \\
\hline $\mathrm{H}$ & 7.524472 & 2.746052 & 0.759342 \\
\hline $\mathrm{C}$ & 5.535544 & 1.136141 & -4.093337 \\
\hline
\end{tabular}




\begin{tabular}{|c|c|c|c|}
\hline $\mathrm{H}$ & 5.565765 & 0.199164 & -3.887183 \\
\hline $\mathrm{H}$ & 5.127773 & 1.261374 & -4.953493 \\
\hline $\mathrm{H}$ & 6.428167 & 1.489960 & -4.108523 \\
\hline $\mathrm{C}$ & 4.728530 & 1.854340 & -3.046576 \\
\hline $\mathrm{C}$ & 4.343239 & 3.171540 & -3.258222 \\
\hline $\mathrm{H}$ & 4.568429 & 3.554026 & -4.076211 \\
\hline $\mathrm{C}$ & 3.654035 & 3.964425 & -2.359129 \\
\hline $\mathrm{C}$ & 3.353974 & 5.399312 & -2.679022 \\
\hline $\mathrm{H}$ & 3.658163 & 5.958330 & -1.959748 \\
\hline $\mathrm{H}$ & 3.804760 & 5.647513 & -3.489741 \\
\hline $\mathrm{H}$ & 2.407504 & 5.510965 & -2.791792 \\
\hline $\mathrm{C}$ & -0.427782 & 0.942258 & -2.072841 \\
\hline $\mathrm{H}$ & -0.073414 & 0.224814 & -2.602765 \\
\hline $\mathrm{H}$ & -1.211384 & 0.640494 & -1.607543 \\
\hline $\mathrm{H}$ & -0.658811 & 1.678562 & -2.644771 \\
\hline $\mathrm{C}$ & 0.621068 & 1.394904 & -1.063078 \\
\hline $\mathrm{C}$ & 0.224520 & 1.957694 & 0.137328 \\
\hline $\mathrm{H}$ & -0.687143 & 2.069347 & 0.281118 \\
\hline $\mathrm{C}$ & 1.093297 & 2.368093 & 1.144021 \\
\hline $\mathrm{C}$ & 0.571139 & 2.893162 & 2.465437 \\
\hline $\mathrm{H}$ & 1.228816 & 3.468777 & 2.862880 \\
\hline $\mathrm{H}$ & -0.238058 & 3.387301 & 2.316800 \\
\hline $\mathrm{H}$ & 0.392031 & 2.156858 & 3.055139 \\
\hline $\mathrm{Cr}$ & 2.119915 & 9.548190 & 7.603111 \\
\hline 0 & 2.262205 & 11.339537 & 8.397835 \\
\hline 0 & 3.779460 & 8.997848 & 8.492187 \\
\hline 0 & 3.187444 & 10.139270 & 6.068271 \\
\hline 0 & 1.943357 & 7.752317 & 6.845223 \\
\hline 0 & 0.519482 & 10.089479 & 6.646825 \\
\hline 0 & 1.065535 & 8.967672 & 9.143282 \\
\hline $\mathrm{C}$ & 3.060843 & 13.227825 & 9.530546 \\
\hline $\mathrm{H}$ & 2.862516 & 13.792124 & 8.779283 \\
\hline $\mathrm{H}$ & 3.871947 & 13.525062 & 9.948992 \\
\hline $\mathrm{H}$ & 2.340116 & 13.271581 & 10.163870 \\
\hline C & 3.231106 & 11.801236 & 9.058139 \\
\hline C & 4.385392 & 11.090581 & 9.369307 \\
\hline $\mathrm{H}$ & 5.070433 & 11.552280 & 9.797124 \\
\hline $\mathrm{C}$ & 4.594583 & 9.749995 & 9.091421 \\
\hline $\mathrm{C}$ & 5.867332 & 9.084605 & 9.556396 \\
\hline $\mathrm{H}$ & 5.673884 & 8.505218 & 10.297966 \\
\hline $\mathrm{H}$ & 6.496934 & 9.754521 & 9.831052 \\
\hline $\mathrm{H}$ & 6.240546 & 8.570098 & 8.837445 \\
\hline $\mathrm{C}$ & 4.251618 & 10.180009 & 3.984767 \\
\hline $\mathrm{H}$ & 4.281838 & 11.116986 & 4.190920 \\
\hline $\mathrm{H}$ & 3.843847 & 10.054776 & 3.124610 \\
\hline $\mathrm{H}$ & 5.144241 & 9.826190 & 3.969580 \\
\hline $\mathrm{C}$ & 3.444604 & 9.461810 & 5.031527 \\
\hline C & 3.059312 & 8.144610 & 4.819881 \\
\hline $\mathrm{H}$ & 3.284502 & 7.762124 & 4.001892 \\
\hline C & 2.370109 & 7.351725 & 5.718974 \\
\hline C & 2.070047 & 5.916838 & 5.399081 \\
\hline $\mathrm{H}$ & 2.374237 & 5.357820 & 6.118355 \\
\hline $\mathrm{H}$ & 2.520833 & 5.668637 & 4.588363 \\
\hline $\mathrm{H}$ & 1.123578 & 5.805185 & 5.286311 \\
\hline C & -1.711709 & 10.373892 & 6.005262 \\
\hline $\mathrm{H}$ & -1.357341 & 11.091336 & 5.475338 \\
\hline $\mathrm{H}$ & -2.495311 & 10.675656 & 6.470561 \\
\hline $\mathrm{H}$ & -1.942738 & 9.637588 & 5.433332 \\
\hline $\mathrm{C}$ & -0.662859 & 9.921246 & 7.015025 \\
\hline $\mathrm{C}$ & -1.059406 & 9.358456 & 8.215431 \\
\hline $\mathrm{H}$ & -1.971070 & 9.246803 & 8.359221 \\
\hline $\mathrm{C}$ & -0.190630 & 8.948057 & 9.222124 \\
\hline $\mathrm{C}$ & -0.712787 & 8.422988 & 10.543540 \\
\hline $\mathrm{H}$ & -0.055111 & 7.847373 & 10.940983 \\
\hline $\mathrm{H}$ & -1.521985 & 7.928849 & 10.394903 \\
\hline $\mathrm{H}$ & -0.891896 & 9.159292 & 11.133242 \\
\hline $\mathrm{Cr}$ & 2.119915 & -5.540010 & 7.603111 \\
\hline 0 & 2.262205 & -3.748663 & 8.397835 \\
\hline 0 & 3.779460 & -6.090352 & 8.492187 \\
\hline 0 & 3.187444 & -4.948930 & 6.068271 \\
\hline
\end{tabular}




\begin{tabular}{|c|c|c|c|}
\hline 0 & 1.943357 & -7.335883 & 6.845223 \\
\hline 0 & 0.519482 & -4.998721 & 6.646825 \\
\hline 0 & 1.065535 & -6.120528 & 9.143282 \\
\hline $\mathrm{C}$ & 3.060843 & -1.860375 & 9.530546 \\
\hline $\mathrm{H}$ & 2.862516 & -1.296076 & 8.77928 \\
\hline $\mathrm{H}$ & 3.871947 & -1.563138 & 9.948992 \\
\hline $\mathrm{H}$ & 2.340116 & -1.816619 & 10.163870 \\
\hline $\mathrm{C}$ & 3.231106 & -3.286964 & 9.058139 \\
\hline $\mathrm{C}$ & 4.385392 & -3.997619 & 9.369307 \\
\hline $\mathrm{H}$ & 5.070433 & -3.535920 & 9.797124 \\
\hline $\mathrm{C}$ & 4.594583 & -5.338205 & 9.091421 \\
\hline $\mathrm{C}$ & 5.867332 & -6.003595 & 9.55639 \\
\hline $\mathrm{H}$ & 5.673884 & -6.582982 & 10.29796 \\
\hline $\mathrm{H}$ & 6.496934 & -5.333679 & 9.831052 \\
\hline $\mathrm{H}$ & 6.240546 & -6.518102 & 8.83744 \\
\hline $\mathrm{C}$ & 4.251618 & -4.908191 & 3.984767 \\
\hline $\mathrm{H}$ & 4.281838 & -3.971214 & 4.190920 \\
\hline $\mathrm{H}$ & 3.843847 & -5.033424 & 3.12461 \\
\hline $\mathrm{H}$ & 5.144241 & -5.262010 & 3.969580 \\
\hline $\mathrm{C}$ & 3.444604 & -5.626390 & 5.031527 \\
\hline $\mathrm{C}$ & 3.059312 & -6.943590 & 4.819881 \\
\hline $\mathrm{H}$ & 3.284502 & -7.326076 & 4.001892 \\
\hline $\mathrm{C}$ & 2.370109 & -7.736475 & 5.718974 \\
\hline $\mathrm{C}$ & 2.070047 & -9.171362 & 5.399081 \\
\hline $\mathrm{H}$ & 2.374237 & -9.730380 & 6.11835 \\
\hline $\mathrm{H}$ & 2.520833 & -9.419563 & 4.588363 \\
\hline $\mathrm{H}$ & 1.123578 & -9.283015 & 5.286311 \\
\hline C & -1.711709 & -4.714308 & 6.005262 \\
\hline $\mathrm{H}$ & -1.357341 & -3.996864 & 5.475338 \\
\hline $\mathrm{H}$ & -2.495311 & -4.412544 & 6.470561 \\
\hline $\mathrm{H}$ & -1.942738 & -5.450612 & 5.433332 \\
\hline $\mathrm{C}$ & -0.662859 & -5.166954 & 7.015025 \\
\hline $\mathrm{C}$ & -1.059406 & -5.729744 & 8.215431 \\
\hline $\mathrm{H}$ & -1.971070 & -5.841397 & 8.359221 \\
\hline C & -0.190630 & -6.140143 & 9.222124 \\
\hline $\mathrm{C}$ & -0.712787 & -6.665212 & 10.543540 \\
\hline $\mathrm{H}$ & -0.055111 & -7.240827 & 10.940983 \\
\hline $\mathrm{H}$ & -1.521985 & -7.159351 & 10.394903 \\
\hline $\mathrm{H}$ & -0.891896 & -5.928908 & 11.133242 \\
\hline $\mathrm{Cr}$ & -4.687768 & 5.540010 & 8.553096 \\
\hline 0 & -4.830058 & 3.748663 & 7.758372 \\
\hline 0 & -6.347313 & 6.090352 & 7.664020 \\
\hline 0 & -5.755298 & 4.948930 & 10.087935 \\
\hline 0 & -4.511210 & 7.335883 & 9.310983 \\
\hline 0 & -3.087335 & 4.998721 & 9.509382 \\
\hline 0 & -3.633388 & 6.120528 & 7.012925 \\
\hline $\mathrm{C}$ & -5.628696 & 1.860375 & 6.625660 \\
\hline $\mathrm{H}$ & -5.430369 & 1.296076 & 7.376924 \\
\hline $\mathrm{H}$ & -6.439800 & 1.563138 & 6.207215 \\
\hline $\mathrm{H}$ & -4.907969 & 1.816619 & 5.992337 \\
\hline $\mathrm{C}$ & -5.798959 & 3.286964 & 7.098068 \\
\hline $\mathrm{C}$ & -6.953245 & 3.997619 & 6.786899 \\
\hline $\mathrm{H}$ & -7.638286 & 3.535920 & 6.359083 \\
\hline $\mathrm{C}$ & -7.162436 & 5.338205 & 7.064786 \\
\hline $\mathrm{C}$ & -8.435185 & 6.003595 & 6.599810 \\
\hline $\mathrm{H}$ & -8.241737 & 6.582982 & 5.858241 \\
\hline $\mathrm{H}$ & -9.064787 & 5.333679 & 6.32515 \\
\hline $\mathrm{H}$ & -8.808399 & 6.518102 & 7. 318762 \\
\hline $\mathrm{C}$ & -6.819471 & 4.908191 & 12.171440 \\
\hline $\mathrm{H}$ & -6.849692 & 3.971214 & 11.965287 \\
\hline $\mathrm{H}$ & -6.411700 & 5.033424 & 13.03159 \\
\hline $\mathrm{H}$ & -7.712094 & 5.262010 & $12.18662^{\circ}$ \\
\hline $\mathrm{C}$ & -6.012457 & 5.626390 & 11.12467 \\
\hline $\mathrm{C}$ & -5.627166 & 6.943590 & 11.33632 \\
\hline $\mathrm{H}$ & -5.852356 & 7.326076 & 12.15431 \\
\hline $\mathrm{C}$ & -4.937962 & 7.736475 & 10.43723 \\
\hline $\mathrm{C}$ & -4.637900 & 9.171362 & 10.75712 \\
\hline $\mathrm{H}$ & -4.942090 & 9.730380 & 10.03785 \\
\hline $\mathrm{H}$ & -5.088686 & 9.419563 & 11.56784 \\
\hline $\mathrm{H}$ & -3.691431 & 9.283015 & 10.86989 \\
\hline
\end{tabular}




\begin{tabular}{|c|c|c|c|}
\hline C & -0.856144 & 4.714308 & 10.150945 \\
\hline $\mathrm{H}$ & -1.210512 & 3.996864 & 10.680868 \\
\hline $\mathrm{H}$ & -0.072542 & 4.412544 & 9.685646 \\
\hline $\mathrm{H}$ & -0.625115 & 5.450612 & 10.722874 \\
\hline $\mathrm{C}$ & -1.904994 & 5.166954 & 9.141182 \\
\hline $\mathrm{C}$ & -1.508447 & 5.729744 & 7.940776 \\
\hline $\mathrm{H}$ & -0.596784 & 5.841397 & 7.796985 \\
\hline C & -2.377224 & 6.140143 & 6.934082 \\
\hline $\mathrm{C}$ & -1.855066 & 6.665212 & 5.612666 \\
\hline $\mathrm{H}$ & -2.512742 & 7.240827 & 5.215223 \\
\hline $\mathrm{H}$ & -1.045869 & 7.159351 & 5.76130 \\
\hline $\mathrm{H}$ & -1.675957 & 5.928908 & 5.022965 \\
\hline $\mathrm{Cr}$ & -4.687768 & -2.004090 & 8.553096 \\
\hline 0 & -4.830058 & -3.795437 & 7.758372 \\
\hline 0 & -6.347313 & -1.453748 & 7.664020 \\
\hline 0 & -5.755298 & -2.595170 & 10.087935 \\
\hline 0 & -4.511210 & -0.208217 & 9.310983 \\
\hline 0 & -3.087335 & -2.545379 & 9.509382 \\
\hline 0 & -3.633388 & -1.423572 & 7.012925 \\
\hline $\mathrm{C}$ & -5.628696 & -5.683725 & 6.625660 \\
\hline $\mathrm{H}$ & -5.430369 & -6.248024 & 7.376924 \\
\hline $\mathrm{H}$ & -6.439800 & -5.980962 & 6.207215 \\
\hline $\mathrm{H}$ & -4.907969 & -5.727481 & 5.992337 \\
\hline C & -5.798959 & -4.257136 & 7.098068 \\
\hline $\mathrm{C}$ & -6.953245 & -3.546481 & 6.786899 \\
\hline $\mathrm{H}$ & -7.638286 & -4.008180 & 6.359083 \\
\hline $\mathrm{C}$ & -7.162436 & -2.205895 & 7.064786 \\
\hline C & -8.435185 & -1.540505 & 6.599810 \\
\hline $\mathrm{H}$ & -8.241737 & -0.961118 & 5.858241 \\
\hline $\mathrm{H}$ & -9.064787 & -2.210421 & 6.325155 \\
\hline $\mathrm{H}$ & -8.808399 & -1.025998 & 7.318762 \\
\hline $\mathrm{C}$ & -6.819471 & -2.635909 & 12.171440 \\
\hline $\mathrm{H}$ & -6.849692 & -3.572886 & 11.965287 \\
\hline $\mathrm{H}$ & -6.411700 & -2.510676 & 13.031596 \\
\hline $\mathrm{H}$ & -7.712094 & -2.282090 & 12.186627 \\
\hline $\mathrm{C}$ & -6.012457 & -1.917710 & 11.124679 \\
\hline $\mathrm{C}$ & -5.627166 & -0.600510 & 11.336325 \\
\hline $\mathrm{H}$ & -5.852356 & -0.218024 & 12.154314 \\
\hline $\mathrm{C}$ & -4.937962 & 0.192375 & 10.437233 \\
\hline C & -4.637900 & 1.627262 & 10.757125 \\
\hline $\mathrm{H}$ & -4.942090 & 2.186280 & 10.037851 \\
\hline $\mathrm{H}$ & -5.088686 & 1.875463 & 11.567844 \\
\hline $\mathrm{H}$ & -3.691431 & 1.738915 & 10.869896 \\
\hline C & -0.856144 & -2.829792 & 10.150945 \\
\hline $\mathrm{H}$ & -1.210512 & -3.547236 & 10.680868 \\
\hline $\mathrm{H}$ & -0.072542 & -3.131556 & 9.685646 \\
\hline $\mathrm{H}$ & -0.625115 & -2.093488 & 10.722874 \\
\hline $\mathrm{C}$ & -1.904994 & -2.377146 & 9.141182 \\
\hline C & -1.508447 & -1.814356 & 7.940776 \\
\hline $\mathrm{H}$ & -0.596784 & -1.702703 & 7.796985 \\
\hline $\mathrm{C}$ & -2.377224 & -1.403957 & 6.934082 \\
\hline $\mathrm{C}$ & -1.855066 & -0.878888 & 5.612666 \\
\hline $\mathrm{H}$ & -2.512742 & -0.303273 & 5.215223 \\
\hline $\mathrm{H}$ & -1.045869 & -0.384749 & 5.761303 \\
\hline $\mathrm{H}$ & -1.675957 & -1.615192 & 5.022965 \\
\hline $\mathrm{Cr}$ & 9.309232 & -2.004090 & 8.553096 \\
\hline 0 & 9.166942 & -3.795437 & 7.758372 \\
\hline 0 & 7.649687 & -1.453748 & 7.664020 \\
\hline 0 & 8.241702 & -2.595170 & 10.087935 \\
\hline 0 & 9.485790 & -0.208217 & 9.310983 \\
\hline 0 & 10.909665 & -2.545379 & 9.509382 \\
\hline 0 & 10.363612 & -1.423572 & 7.012925 \\
\hline $\mathrm{C}$ & 8.368304 & -5.683725 & 6.625660 \\
\hline $\mathrm{H}$ & 8.566631 & -6.248024 & 7.376924 \\
\hline $\mathrm{H}$ & 7.557200 & -5.980962 & 6.207215 \\
\hline $\mathrm{H}$ & 9.089031 & -5.727481 & 5.992337 \\
\hline $\mathrm{C}$ & 8.198041 & -4.257136 & 7.098068 \\
\hline $\mathrm{C}$ & 7.043755 & -3.546481 & 6.786899 \\
\hline $\mathrm{H}$ & 6.358714 & -4.008180 & 6.359083 \\
\hline$C$ & 6.834564 & -2.205895 & $7.0647 \varepsilon$ \\
\hline
\end{tabular}




\begin{tabular}{|c|c|c|c|}
\hline $\mathrm{C}$ & 5.561815 & -1.540505 & 6.599810 \\
\hline $\mathrm{H}$ & 5.755263 & -0.961118 & 5.858241 \\
\hline $\mathrm{H}$ & 4.932213 & -2.210421 & 6.325155 \\
\hline $\mathrm{H}$ & 5.188601 & -1.025998 & 7.318762 \\
\hline C & 7.177529 & -2.635909 & 12.171440 \\
\hline $\mathrm{H}$ & 7.147308 & -3.572886 & 11.965287 \\
\hline $\mathrm{H}$ & 7.585300 & -2.510676 & 13.031596 \\
\hline $\mathrm{H}$ & 6.284906 & -2.282090 & 12.186627 \\
\hline $\mathrm{C}$ & 7.984543 & -1.917710 & 11.124679 \\
\hline $\mathrm{C}$ & 8.369834 & -0.600510 & 11.336325 \\
\hline $\mathrm{H}$ & 8.144644 & -0.218024 & 12.154314 \\
\hline $\mathrm{C}$ & 9.059038 & 0.192375 & 10.437233 \\
\hline $\mathrm{C}$ & 9.359100 & 1.627262 & 10.757125 \\
\hline $\mathrm{H}$ & 9.054910 & 2.186280 & 10.037851 \\
\hline $\mathrm{H}$ & 8.908314 & 1.875463 & 11.567844 \\
\hline $\mathrm{H}$ & 10.305569 & 1.738915 & 10.869896 \\
\hline $\mathrm{C}$ & 13.140856 & -2.829792 & 10.150945 \\
\hline $\mathrm{H}$ & 12.786488 & -3.547236 & 10.680868 \\
\hline $\mathrm{H}$ & 13.924458 & -3.131556 & 9.685646 \\
\hline $\mathrm{H}$ & 13.371885 & -2.093488 & 10.722874 \\
\hline $\mathrm{C}$ & 12.092006 & -2.377146 & 9.141182 \\
\hline $\mathrm{C}$ & 12.488553 & -1.814356 & 7.940776 \\
\hline $\mathrm{H}$ & 13.400216 & -1.702703 & 7.79698 \\
\hline $\mathrm{C}$ & 11.619776 & -1.403957 & 6.934082 \\
\hline $\mathrm{C}$ & 12.141934 & -0.878888 & 5.612666 \\
\hline $\mathrm{H}$ & 11.484258 & -0.303273 & 5.21522 \\
\hline $\mathrm{H}$ & 12.951131 & -0.384749 & 5.761303 \\
\hline $\mathrm{H}$ & 12.321043 & -1.615192 & 5.02296 \\
\hline $\mathrm{Cr}$ & 9.309232 & 5.540010 & 8.553096 \\
\hline 0 & 9.166942 & 3.748663 & 7.758372 \\
\hline 0 & 7.649687 & 6.090352 & 7.664020 \\
\hline 0 & 8.241702 & 4.948930 & 10.08793 \\
\hline 0 & 9.485790 & 7.335883 & 9.31098 \\
\hline 0 & 10.909665 & 4.998721 & 9.509382 \\
\hline 0 & 10.363612 & 6.120528 & 7.01292 \\
\hline $\mathrm{C}$ & 8.368304 & 1.860375 & 6.625660 \\
\hline $\mathrm{H}$ & 8.566631 & 1.296076 & 7.376924 \\
\hline $\mathrm{H}$ & 7.557200 & 1.563138 & 6.20721 \\
\hline $\mathrm{H}$ & 9.089031 & 1.816619 & 5.992337 \\
\hline $\mathrm{C}$ & 8.198041 & 3.286964 & 7.098068 \\
\hline $\mathrm{C}$ & 7.043755 & 3.997619 & 6.786899 \\
\hline $\mathrm{H}$ & 6.358714 & 3.535920 & 6.359083 \\
\hline $\mathrm{C}$ & 6.834564 & 5.338205 & 7.064786 \\
\hline $\mathrm{C}$ & 5.561815 & 6.003595 & 6.599810 \\
\hline $\mathrm{H}$ & 5.755263 & 6.582982 & 5.858241 \\
\hline $\mathrm{H}$ & 4.932213 & 5.333679 & 6.32515 \\
\hline $\mathrm{H}$ & 5.188601 & 6.518102 & 7. 318762 \\
\hline $\mathrm{C}$ & 7.177529 & 4.908191 & 12.171440 \\
\hline $\mathrm{H}$ & 7.147308 & 3.971214 & 11.965287 \\
\hline $\mathrm{H}$ & 7.585300 & 5.033424 & 13.03159 \\
\hline $\mathrm{H}$ & 6.284906 & 5.262010 & 12.186627 \\
\hline $\mathrm{C}$ & 7.984543 & 5.626390 & 11.12467 \\
\hline $\mathrm{C}$ & 8.369834 & 6.943590 & 11.336325 \\
\hline $\mathrm{H}$ & 8.144644 & 7.326076 & 12.154314 \\
\hline $\mathrm{C}$ & 9.059038 & 7.736475 & 10.43723 \\
\hline $\mathrm{C}$ & 9.359100 & 9.171362 & 10.75712 \\
\hline $\mathrm{H}$ & 9.054910 & 9.730380 & 10.03785 \\
\hline $\mathrm{H}$ & 8.908314 & 9.419563 & 11.56784 \\
\hline $\mathrm{H}$ & 10.305569 & 9.283015 & 10.86989 \\
\hline $\mathrm{C}$ & 13.140856 & 4.714308 & 10.15094 \\
\hline $\mathrm{H}$ & 12.786488 & 3.996864 & 10.680868 \\
\hline $\mathrm{H}$ & 13.924458 & 4.412544 & 9.68564 \\
\hline $\mathrm{H}$ & 13.371885 & 5.450612 & 10.72287 \\
\hline $\mathrm{C}$ & 12.092006 & 5.166954 & 9.14118 \\
\hline $\mathrm{C}$ & 12.488553 & 5.729744 & 7.94077 \\
\hline $\mathrm{H}$ & 13.400216 & 5.841397 & 7.79698 \\
\hline $\mathrm{C}$ & 11.619776 & 6.140143 & 6.93408 \\
\hline $\mathrm{C}$ & 12.141934 & 6.665212 & 5.61266 \\
\hline $\mathrm{H}$ & 11.484258 & 7.240827 & 5.21522 \\
\hline $\mathrm{H}$ & 12.951131 & 7.159351 & 5.76130 \\
\hline
\end{tabular}




\section{Compound 1a with Ga atoms in embedding - optimized central molecule}

\begin{tabular}{|c|c|c|c|}
\hline $\mathrm{Cr}$ & 2.0781876 & 2.0080092 & 7.59701 \\
\hline 0 & 2.2201109 & 3.8076483 & 8.3880228 \\
\hline 0 & 3.7954402 & 1.5056508 & 8.4381697 \\
\hline 0 & 3.1022528 & 2.6076571 & 6.0168878 \\
\hline 0 & 1.9139972 & 0.2028129 & 6.8216153 \\
\hline 0 & 0.4047156 & 2.5271018 & 6.7079205 \\
\hline 0 & 1.0668326 & 1.4115519 & 9.1717919 \\
\hline $\mathrm{C}$ & 3.0486809 & 5.7281026 & 9.4370776 \\
\hline $\mathrm{H}$ & 2.7029315 & 6.3549873 & 8.6140063 \\
\hline $\mathrm{H}$ & 3.9644334 & 6.1463883 & 9.8484122 \\
\hline $\mathrm{H}$ & 2.2664899 & 5.7613774 & 10.2000217 \\
\hline $\mathrm{C}$ & 3.2199797 & 4.3120911 & 8.9876778 \\
\hline $\mathrm{C}$ & 4.4125248 & 3.6391236 & 9.2551295 \\
\hline $\mathrm{H}$ & 5.2169072 & 4.1918741 & 9.7175380 \\
\hline C & 4.6327903 & 2.2854728 & 8.9833047 \\
\hline $\mathrm{C}$ & 5.9307365 & 1.6664475 & 9.3975033 \\
\hline $\mathrm{H}$ & 5.7635890 & 1.0424277 & 10.2798596 \\
\hline $\mathrm{H}$ & 6.6761101 & 2.4221906 & 9.6351672 \\
\hline $\mathrm{H}$ & 6.3055402 & 1.0058394 & 8.6151925 \\
\hline $\mathrm{C}$ & 4.1998198 & 2.6236973 & 3.9375999 \\
\hline $\mathrm{H}$ & 4.3504258 & 3.6662567 & 4.2091151 \\
\hline $\mathrm{H}$ & 3.7100784 & 2.5577147 & 2.9635589 \\
\hline $\mathrm{H}$ & 5.1714768 & 2.1326115 & 3.8462185 \\
\hline $\mathrm{C}$ & 3.3914387 & 1.9293684 & 4.9898442 \\
\hline $\mathrm{C}$ & 3.0315172 & 0.5926044 & 4.7756886 \\
\hline $\mathrm{H}$ & 3.3371400 & 0.1440453 & 3.8410477 \\
\hline $\mathrm{C}$ & 2.3318272 & -0.1937550 & 5.6898899 \\
\hline $\mathrm{C}$ & 2.0150874 & -1.6188100 & 5.3566691 \\
\hline $\mathrm{H}$ & 2.3034995 & -2.2796747 & 6.1749560 \\
\hline $\mathrm{H}$ & 2.4963022 & -1.9422204 & 4.4357266 \\
\hline $\mathrm{H}$ & 0.9345080 & -1.7263341 & 5.2435587 \\
\hline $\mathrm{C}$ & -1.8699058 & 2.7840164 & 6.1998164 \\
\hline $\mathrm{H}$ & -1.5145373 & 3.6149857 & 5.5943162 \\
\hline $\mathrm{H}$ & -2.7725353 & 3.0636113 & 6.7405859 \\
\hline $\mathrm{H}$ & -2.1080156 & 1.9586970 & 5.5245501 \\
\hline $\mathrm{C}$ & -0.7790871 & 2.3475202 & 7.1269042 \\
\hline $\mathrm{C}$ & -1.1230797 & 1.7661823 & 8.3480328 \\
\hline $\mathrm{H}$ & -2.1738885 & 1.6482430 & 8.5725841 \\
\hline $\mathrm{C}$ & -0.1941773 & 1.3522111 & 9.3021883 \\
\hline $\mathrm{C}$ & -0.6797954 & 0.8394286 & 10.6225959 \\
\hline $\mathrm{H}$ & 0.0265044 & 0.1131841 & 11.0209956 \\
\hline $\mathrm{H}$ & -1.6710618 & 0.3964317 & 10.5403757 \\
\hline $\mathrm{H}$ & -0.7191816 & 1.6653817 & 11.3392470 \\
\hline $\mathrm{Ga}$ & 0.8359880 & 1.7679600 & 15.6812140 \\
\hline 0 & 0.9782780 & -0.0233870 & 16.4759380 \\
\hline 0 & 2.4955330 & 2.3183020 & 16.5702900 \\
\hline 0 & 1.9035180 & 1.1768800 & 14.1463740 \\
\hline 0 & 0.6594300 & 3.5638330 & 14.9233260 \\
\hline 0 & -0.7644450 & 1.2266710 & 14.7249280 \\
\hline 0 & -0.2183920 & 2.3484780 & 17.2213850 \\
\hline $\mathrm{C}$ & 1.7769160 & -1.9116750 & 17.6086500 \\
\hline $\mathrm{H}$ & 1.5277575 & -2.5436650 & 16.759152 \\
\hline $\mathrm{H}$ & 2.6682573 & -2.2974046 & 18.1283462 \\
\hline $\mathrm{H}$ & 0.9196862 & -1.9461376 & 18.309219 \\
\hline $\mathrm{C}$ & 1.9471800 & -0.4850860 & 17.1362420 \\
\hline $\mathrm{C}$ & 3.1014650 & 0.2255690 & 17.447411 \\
\hline $\mathrm{H}$ & 3.8812733 & -0.2969409 & 18.004155 \\
\hline $\mathrm{C}$ & 3.3106570 & 1.5661550 & 17.169524 \\
\hline C & 4.5834050 & 2.2315450 & 17.634499 \\
\hline $\mathrm{H}$ & 4.3388794 & 2.9650616 & 18.415955 \\
\hline $\mathrm{H}$ & 5.3223544 & 1.5200451 & 18.020310 \\
\hline $\mathrm{H}$ & 5.0166169 & 2.7981031 & 16.790700 \\
\hline C & 2.9676910 & 1.1361410 & 12.062870 \\
\hline $\mathrm{H}$ & 3.0254007 & 0.0695557 & 12.319446 \\
\hline
\end{tabular}




\begin{tabular}{|c|c|c|c|}
\hline $\mathrm{H}$ & 2.5362860 & 1.2669439 & 11.0638581 \\
\hline $\mathrm{H}$ & 3.9852333 & 1.5552690 & 12.041040 \\
\hline $\mathrm{C}$ & 2.1606770 & 1.8543400 & 13.109631 \\
\hline C & 1.7753860 & 3.1715400 & 12.897984 \\
\hline $\mathrm{H}$ & 2.0788614 & 3.6195015 & 11.957602 \\
\hline C & 1.0861820 & 3.9644250 & 13.79707 \\
\hline C & 0.7861210 & 5.3993120 & 13.477184 \\
\hline $\mathrm{H}$ & 1.1315324 & 6.0248241 & 14.322998 \\
\hline $\mathrm{H}$ & 1.2559710 & 5.7326380 & 12.55384 \\
\hline $\mathrm{H}$ & -0.3038024 & 5.5267783 & 13.41554 \\
\hline C & -2.9956350 & 0.9422580 & 14.083365 \\
\hline $\mathrm{H}$ & -2.5850808 & 0.1042994 & 13.482252 \\
\hline $\mathrm{H}$ & -3.9325954 & 0.6207927 & 14.55981 \\
\hline $\mathrm{H}$ & -3.2167685 & 1.7655218 & 13.401035 \\
\hline $\mathrm{C}$ & -1.9467850 & 1.3949040 & $15.09312 \varepsilon$ \\
\hline $\mathrm{C}$ & -2.3433330 & 1.9576940 & 16.293534 \\
\hline $\mathrm{H}$ & -3.4184172 & 2.0219062 & 16.489704 \\
\hline $\mathrm{C}$ & -1.4745560 & 2.3680930 & 17.300228 \\
\hline $\mathrm{C}$ & -1.9967140 & 2.8931620 & 18.6216440 \\
\hline $\mathrm{H}$ & -1.2717604 & 3.5857438 & 19.043340 \\
\hline $\mathrm{H}$ & -2.9849535 & 3.3569864 & 18.5290332 \\
\hline $\mathrm{H}$ & -2.1004275 & 2.0353204 & 19.328517 \\
\hline $\mathrm{Ga}$ & 3.4038420 & 1.7679600 & -0.474992 \\
\hline 0 & 3.5461310 & -0.0233870 & 0.319731 \\
\hline 0 & 5.0633870 & 2.3183020 & 0.414084 \\
\hline 0 & 4.4713710 & 1.1768800 & -2.009832 \\
\hline 0 & 3.2272830 & 3.5638330 & -1.232880 \\
\hline 0 & 1.8034080 & 1.2266710 & -1.431278 \\
\hline 0 & 2.3494620 & 2.3484780 & 1.0651790 \\
\hline $\mathrm{C}$ & 4.3447690 & -1.9116750 & 1.452443 \\
\hline $\mathrm{H}$ & 4.1077688 & -2.5432321 & $0.578518^{\circ}$ \\
\hline $\mathrm{H}$ & 5.2348017 & -2.2997331 & 1.960974 \\
\hline $\mathrm{H}$ & 3.4816996 & -1.9573886 & 2.124259 \\
\hline $\mathrm{C}$ & 4.5150330 & -0.4850860 & 0.980035 \\
\hline $\mathrm{C}$ & 5.6693180 & 0.2255690 & 1.2912040 \\
\hline $\mathrm{H}$ & 6.4488492 & -0.2983289 & 1.843616 \\
\hline $\mathrm{C}$ & 5.8785100 & 1.5661550 & 1.013317 \\
\hline C & 7.1512580 & 2.2315450 & 1.4782930 \\
\hline $\mathrm{H}$ & 6.9099432 & 2.9601445 & 2.2692861 \\
\hline $\mathrm{H}$ & 7.8905793 & 1.5194145 & $1.860129^{\circ}$ \\
\hline $\mathrm{H}$ & 7.5815217 & 2.8075474 & 0.6410879 \\
\hline $\mathrm{C}$ & 5.5355440 & 1.1361410 & -4.093337 \\
\hline $\mathrm{H}$ & 5.5574556 & 0.0678321 & -3.867057 \\
\hline $\mathrm{H}$ & 5.1696788 & 1.3283660 & -5.092930 \\
\hline $\mathrm{H}$ & 6.5976757 & 1.5093711 & -4.055965 \\
\hline $\mathrm{C}$ & 4.7285300 & 1.8543400 & -3.046576 \\
\hline C & 4.3432390 & 3.1715400 & -3.258222 \\
\hline $\mathrm{H}$ & 4.6718536 & 3.6366059 & -4.191634 \\
\hline $\mathrm{C}$ & 3.6540350 & 3.9644250 & -2.359129 \\
\hline $\mathrm{C}$ & 3.3539740 & 5.3993120 & -2.679022 \\
\hline $\mathrm{H}$ & 3.7505823 & 6.0343815 & -1.847141 \\
\hline $\mathrm{H}$ & 3.8204894 & 5.7380668 & -3.617955 \\
\hline $\mathrm{H}$ & 2.2854051 & 5.5493735 & -2.707796 \\
\hline $\mathrm{C}$ & -0.4277820 & 0.9422580 & -2.072841 \\
\hline $\mathrm{H}$ & -0.0040551 & 0.1475356 & -2.696833 \\
\hline $\mathrm{H}$ & -1.3570160 & 0.6424817 & -1.610603 \\
\hline $\mathrm{H}$ & -0.6498433 & 1.8060503 & -2.760593 \\
\hline $\mathrm{C}$ & 0.6210680 & 1.3949040 & -1.063078 \\
\hline $\mathrm{C}$ & 0.2245200 & 1.9576940 & 0.137328 \\
\hline $\mathrm{H}$ & -0.8458970 & 2.0588455 & 0.317222 \\
\hline $\mathrm{C}$ & 1.0932970 & 2.3680930 & 1.144021 \\
\hline $\mathrm{C}$ & 0.5711390 & 2.8931620 & 2.465437 \\
\hline $\mathrm{H}$ & 1.2577896 & 3.6739890 & 2.839009 \\
\hline $\mathrm{H}$ & -0.4447371 & 3.3282360 & 2.369153 \\
\hline $\mathrm{H}$ & 0.5450800 & 2.0925261 & 3.192104 \\
\hline $\mathrm{Ga}$ & 2.1199150 & 9.5481900 & 7.603111 \\
\hline 0 & 2.2622050 & 11.3395370 & 8.397835 \\
\hline 0 & 3.7794600 & 8.9978480 & $8.492187 C$ \\
\hline O & 3.1874440 & 10.1392700 & 6.068271 \\
\hline 0 & 1.94 & 7.7523170 & 6.845223 \\
\hline
\end{tabular}




\begin{tabular}{|c|c|c|c|}
\hline $\mathrm{O}$ & 0.5194820 & 10.0894790 & 6.6468250 \\
\hline 0 & 1.0655350 & 8.9676720 & 9.1432820 \\
\hline $\mathrm{C}$ & 3.0608430 & 13.2278250 & 9.5305460 \\
\hline $\mathrm{H}$ & 2.7899781 & 13.8612300 & 8.6721104 \\
\hline & 3.9530163 & 13.6233237 & 10.0242265 \\
\hline & 2.2086670 & 13.2625698 & 10.2325418 \\
\hline & 3.2311060 & 11.8012360 & 9.0581390 \\
\hline C & 4.3853920 & 11.0905810 & 9.369307 \\
\hline $\mathrm{H}$ & 5.1646866 & 11.6203446 & 9.9106890 \\
\hline 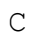 & 4.5945830 & 9.7499950 & 9.091421 \\
\hline C & 5.8673320 & 9.0846050 & 9.55639 \\
\hline $\mathrm{H}$ & 5.6368813 & 8.4013616 & 10.367890 \\
\hline $\mathrm{H}$ & 6.6108690 & 9.8184402 & 9.895577 \\
\hline $\mathrm{H}$ & 6.3053794 & 8.4782710 & 8.7377975 \\
\hline C & 4.2516180 & 10.1800090 & 3.9847670 \\
\hline $\mathrm{H}$ & 4.2536676 & 11.2626114 & 4.2036101 \\
\hline $\mathrm{H}$ & 3.8412632 & 10.0157018 & 2.9715109 \\
\hline $\mathrm{H}$ & 5.2811881 & 9.8155615 & 3.9886161 \\
\hline $\mathrm{C}$ & 3.4446040 & 9.4618100 & 5.0315270 \\
\hline $\mathrm{C}$ & 3.0593120 & 8.1446100 & 4.8198810 \\
\hline $\mathrm{H}$ & 3.3604961 & 7.6926281 & 3.8696601 \\
\hline $\mathrm{C}$ & 2.3701090 & 7.3517250 & 5.71897 \\
\hline $\mathrm{C}$ & 2.0700470 & 5.9168380 & 5.3990810 \\
\hline $\mathrm{H}$ & 2.3652282 & 5.2713962 & 6.2247961 \\
\hline $\mathrm{H}$ & 2.5598138 & 5.5792929 & 4.468328 \\
\hline $\mathrm{H}$ & 0.9834464 & 5.7867884 & 5.28026 \\
\hline $\mathrm{C}$ & -1.7117090 & 10.3738920 & 6.005262 \\
\hline $\mathrm{H}$ & -1.2946305 & 11.1744106 & 5.389087 \\
\hline $\mathrm{H}$ & -2.6401582 & 10.7089090 & 6.4954163 \\
\hline $\mathrm{H}$ & -1.9840907 & 9.5318265 & 5.339969 \\
\hline $\mathrm{C}$ & -0.6628590 & 9.9212460 & 7.015025 \\
\hline $\mathrm{C}$ & -1.0594060 & 9.3584560 & 8.2154310 \\
\hline $\mathrm{H}$ & -2.1324035 & 9.2346559 & 8.3952048 \\
\hline $\mathrm{C}$ & -0.1906300 & 8.9480570 & 9.2221240 \\
\hline $\mathrm{C}$ & -0.7127870 & 8.4229880 & 10.543540 \\
\hline $\mathrm{H}$ & 0.0687911 & 7.8070235 & 11.0085866 \\
\hline $\mathrm{H}$ & -1.6356494 & 7.8370539 & 10.4067170 \\
\hline $\mathrm{H}$ & -0.9264863 & 9.2660927 & 11.2045691 \\
\hline $\mathrm{Ga}$ & 2.1199150 & -5.5400100 & 7.6031110 \\
\hline 0 & 2.2622050 & -3.7486630 & 8.397835 \\
\hline O & 3.7794600 & -6.0903520 & 8.4921870 \\
\hline 0 & 3.1874440 & -4.9489300 & 6.0682710 \\
\hline 0 & 1.9433570 & -7.3358830 & 6.8452230 \\
\hline 0 & 0.5194820 & -4.9987210 & 6.646825 \\
\hline 0 & 1.0655350 & -6.1205280 & 9.1432820 \\
\hline $\mathrm{C}$ & 3.0608430 & -1.8603750 & 9.5305460 \\
\hline $\mathrm{H}$ & 2.8044920 & -1.2139625 & 8.6904302 \\
\hline $\mathrm{H}$ & 3.9764502 & -1.4730684 & 10.0245592 \\
\hline $\mathrm{H}$ & 2.2336438 & -1.8269321 & 10.2411058 \\
\hline $\mathrm{C}$ & 3.2311060 & -3.2869640 & 9.0581390 \\
\hline C & 4.3853920 & -3.9976190 & 9.3693070 \\
\hline $\mathrm{H}$ & 5.1908811 & -3.4757163 & 9.8902018 \\
\hline C & 4.5945830 & -5.3382050 & 9.0914210 \\
\hline $\mathrm{C}$ & 5.8673320 & -6.0035950 & 9.5563960 \\
\hline $\mathrm{H}$ & 5.6379454 & -6.6766538 & 10.4003805 \\
\hline $\mathrm{H}$ & 6.6394397 & -5.2885536 & 9.851144 \\
\hline $\mathrm{H}$ & 6.2544613 & -6.6514006 & 8.7417806 \\
\hline C & 4.2516180 & -4.9081910 & 3.9847670 \\
\hline $\mathrm{H}$ & 4.2692778 & -3.8440393 & 4.2125446 \\
\hline $\mathrm{H}$ & 3.8520042 & -5.0902886 & 2.9851188 \\
\hline $\mathrm{H}$ & 5.2879594 & -5.2947503 & 4.005324 \\
\hline C & 3.4446040 & -5.6263900 & 5.0315270 \\
\hline C & 3.0593120 & -6.9435900 & 4.8198810 \\
\hline $\mathrm{H}$ & 3.3527270 & -7.4042969 & 3.8835441 \\
\hline C & 2.3701090 & -7.7364750 & 5.7189740 \\
\hline C & 2.0700470 & -9.1713620 & 5.399081 \\
\hline $\mathrm{H}$ & 2.3649792 & -9.8016424 & 6.244322 \\
\hline $\mathrm{H}$ & 2.5379822 & -9.5141667 & 4.476128 \\
\hline $\mathrm{H}$ & 0.9619616 & -9.2894488 & 5.301095 \\
\hline & & -4.7143080 & \\
\hline
\end{tabular}




\begin{tabular}{|c|c|c|c|}
\hline $\mathrm{H}$ & -1.2765978 & -3.9320440 & 5.3734902 \\
\hline 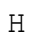 & -2.6237235 & -4.3324295 & 6.5088356 \\
\hline $\mathrm{H}$ & -1.9809905 & -5.5637504 & 5.3826504 \\
\hline $\mathrm{C}$ & -0.6628590 & -5.1669540 & 7.0150250 \\
\hline $\mathrm{C}$ & -1.0594060 & -5.7297440 & 8.2154310 \\
\hline 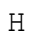 & -2.1339684 & -5.7636214 & 8.4306103 \\
\hline $\mathrm{C}$ & -0.1906300 & -6.1401430 & 9.2221240 \\
\hline $\mathrm{C}$ & -0.7127870 & -6.6652120 & 10.5435400 \\
\hline $\mathrm{H}$ & 0.0500874 & -7.2825509 & 11.0177613 \\
\hline $\mathrm{H}$ & -1.6566525 & -7.2087672 & 10.4363566 \\
\hline $\mathrm{H}$ & -0.9236860 & -5.8029280 & 11.2318475 \\
\hline $\mathrm{Ga}$ & -4.6877680 & 5.5400100 & 8.553096 \\
\hline 0 & -4.8300580 & 3.7486630 & 7.7583720 \\
\hline 0 & -6.3473130 & 6.0903520 & 7.6640200 \\
\hline O & -5.7552980 & 4.9489300 & 10.0879350 \\
\hline 0 & -4.5112100 & 7.3358830 & 9.3109830 \\
\hline 0 & -3.0873350 & 4.9987210 & 9.5093820 \\
\hline 0 & -3.6333880 & 6.1205280 & 7.0129250 \\
\hline $\mathrm{C}$ & -5.6286960 & 1.8603750 & 6.6256600 \\
\hline $\mathrm{H}$ & -5.3577250 & 1.2078651 & 7.4769165 \\
\hline $\mathrm{H}$ & -6.5310015 & 1.4646134 & 6.1451247 \\
\hline $\mathrm{H}$ & -4.7917121 & 1.8194851 & 5.9218684 \\
\hline $\mathrm{C}$ & -5.7989590 & 3.2869640 & 7.0980680 \\
\hline $\mathrm{C}$ & -6.9532450 & 3.9976190 & 6.7868990 \\
\hline $\mathrm{H}$ & -7.7354839 & 3.4657310 & 6.2475943 \\
\hline $\mathrm{C}$ & -7.1624360 & 5.3382050 & 7.0647860 \\
\hline C & -8.4351850 & 6.0035950 & 6.5998100 \\
\hline $\mathrm{H}$ & -8.2028796 & 6.7122522 & 5.8115533 \\
\hline $\mathrm{H}$ & -9.1866899 & 5.2797383 & 6.2486388 \\
\hline $\mathrm{H}$ & -8.8625263 & 6.5827842 & 7.4474369 \\
\hline C & -6.8194710 & 4.9081910 & 12.1714400 \\
\hline $\mathrm{H}$ & -6.8518950 & 3.8362444 & 11.9346492 \\
\hline $\mathrm{H}$ & -6.4296330 & 5.0742629 & 13.1722345 \\
\hline $\mathrm{H}$ & -7.8647391 & 5.2912820 & 12.1526250 \\
\hline C & -6.0124570 & 5.6263900 & 11.1246790 \\
\hline C & -5.6271660 & 6.9435900 & 11.3363250 \\
\hline $\mathrm{H}$ & -5.9331567 & 7.3948911 & 12.2808510 \\
\hline C & -4.9379620 & 7.7364750 & 10.4372330 \\
\hline C & -4.6379000 & 9.1713620 & 10.7571250 \\
\hline $\mathrm{H}$ & -4.9573435 & 9.7968555 & 9.9280926 \\
\hline $\mathrm{H}$ & -5.1159166 & 9.5055532 & 11.6952127 \\
\hline $\mathrm{H}$ & -3.5444991 & 9.2931770 & 10.8616423 \\
\hline C & -0.8561440 & 4.7143080 & 10.1509450 \\
\hline $\mathrm{H}$ & -1.2828114 & 3.9222383 & 10.7723879 \\
\hline $\mathrm{H}$ & 0.0620195 & 4.3535325 & 9.6489237 \\
\hline $\mathrm{H}$ & -0.5901591 & 5.5580703 & 10.7893056 \\
\hline C & -1.9049940 & 5.1669540 & 9.1411820 \\
\hline C & -1.5084470 & 5.7297440 & 7.9407760 \\
\hline $\mathrm{H}$ & -0.4384986 & 5.8167904 & 7.7449885 \\
\hline $\mathrm{C}$ & -2.3772240 & 6.1401430 & 6.9340820 \\
\hline $\mathrm{C}$ & -1.8550660 & 6.6652120 & 5.6126660 \\
\hline $\mathrm{H}$ & -2.6341993 & 7.2854975 & 5.1433807 \\
\hline $\mathrm{H}$ & -0.9288328 & 7.2304423 & 5.7391953 \\
\hline $\mathrm{H}$ & -1.6507657 & 5.8159525 & 4.9319147 \\
\hline $\mathrm{Ga}$ & -4.6877680 & -2.0040900 & 8.5530960 \\
\hline 0 & -4.8300580 & -3.7954370 & 7.7583720 \\
\hline 0 & -6.3473130 & -1.4537480 & 7.6640200 \\
\hline 0 & -5.7552980 & -2.5951700 & 10.0879350 \\
\hline 0 & -4.5112100 & -0.2082170 & 9.3109830 \\
\hline 0 & -3.0873350 & -2.5453790 & 9.5093820 \\
\hline 0 & -3.6333880 & -1.4235720 & 7.0129250 \\
\hline $\mathrm{C}$ & -5.6286960 & -5.6837250 & 6.6256600 \\
\hline $\mathrm{H}$ & -5.4440431 & -6.3279313 & 7.499461 \\
\hline $\mathrm{H}$ & -6.5151354 & -6.0606645 & 6.080884 \\
\hline $\mathrm{H}$ & -4.7515786 & -5.7411722 & 5.980723 \\
\hline $\mathrm{C}$ & -5.7989590 & -4.2571360 & 7.0980680 \\
\hline $\mathrm{C}$ & -6.9532450 & -3.5464810 & 6.786899 \\
\hline $\mathrm{H}$ & -7.7429641 & -4.0730959 & 6.2408589 \\
\hline $\mathrm{C}$ & -7.1624360 & -2.2058950 & 7.064786 \\
\hline $\mathrm{C}$ & -8.4351850 & -1.5405050 & 6.599810 \\
\hline
\end{tabular}




\begin{tabular}{|c|c|c|c|}
\hline $\mathrm{H}$ & -8.1984404 & -0.8289755 & 5.8050925 \\
\hline $\mathrm{H}$ & -9.1890686 & -2.2565801 & 6.2390217 \\
\hline $\mathrm{H}$ & -8.8633656 & -0.9557243 & 7.4397932 \\
\hline C & -6.8194710 & -2.6359090 & 12.1714400 \\
\hline $\mathrm{H}$ & -6.8164271 & -3.7128378 & 11.961549 \\
\hline $\mathrm{H}$ & -6.4216580 & -2.4510925 & 13.183511 \\
\hline $\mathrm{H}$ & -7.8584943 & -2.2732162 & 12.156990 \\
\hline $\mathrm{C}$ & -6.0124570 & -1.9177100 & 11.124675 \\
\hline C & -5.6271660 & -0.6005100 & 11.336325 \\
\hline $\mathrm{H}$ & -5.9255266 & -0.1436645 & 12.282291 \\
\hline C & -4.9379620 & 0.1923750 & 10.437233 \\
\hline $\mathrm{C}$ & -4.6379000 & 1.6272620 & 10.757125 \\
\hline $\mathrm{H}$ & -4.9687150 & 2.2754787 & 9.937642 \\
\hline $\mathrm{H}$ & -5.0898779 & 1.9554636 & 11.697760 \\
\hline $\mathrm{H}$ & -3.5415299 & 1.7620241 & 10.823568 \\
\hline $\mathrm{C}$ & -0.8561440 & -2.8297920 & 10.150945 \\
\hline $\mathrm{H}$ & -1.2934176 & -3.6158926 & 10.783730 \\
\hline $\mathrm{H}$ & 0.0565494 & -3.1806629 & 9.663271 \\
\hline $\mathrm{H}$ & -0.6011491 & -1.9804427 & 10.807198 \\
\hline $\mathrm{C}$ & -1.9049940 & -2.3771460 & 9.141182 \\
\hline $\mathrm{C}$ & -1.5084470 & -1.8143560 & 7.940776 \\
\hline $\mathrm{H}$ & -0.4403959 & -1.7330545 & 7.754323 \\
\hline $\mathrm{C}$ & -2.3772240 & -1.4039570 & 6.934082 \\
\hline $\mathrm{C}$ & -1.8550660 & -0.8788880 & 5.612666 \\
\hline $\mathrm{H}$ & -2.6391449 & -0.2724099 & 5.146055 \\
\hline $\mathrm{H}$ & -0.9384069 & -0.2947449 & 5.756679 \\
\hline $\mathrm{H}$ & -1.6259520 & -1.7227737 & 4.946320 \\
\hline $\mathrm{Ga}$ & 9.3092320 & -2.0040900 & 8.553096 \\
\hline 0 & 9.1669420 & -3.7954370 & 7.758372 \\
\hline 0 & 7.6496870 & -1.4537480 & 7.664020 \\
\hline 0 & 8.2417020 & -2.5951700 & 10.087935 \\
\hline 0 & 9.4857900 & -0.2082170 & 9.310983 \\
\hline 0 & 10.9096650 & -2.5453790 & 9.5093820 \\
\hline 0 & 10.3636120 & -1.4235720 & 7.012925 \\
\hline $\mathrm{C}$ & 8.3683040 & -5.6837250 & $6.625660 \mathrm{c}$ \\
\hline $\mathrm{H}$ & 8.6565343 & -6.3156482 & 7.474820 \\
\hline $\mathrm{H}$ & 7.4671612 & -6.0855822 & 6.146077 \\
\hline $\mathrm{H}$ & 9.2053754 & -5.7168175 & 5.9021372 \\
\hline $\mathrm{C}$ & 8.1980410 & -4.2571360 & 7.098068 \\
\hline C & 7.0437550 & -3.5464810 & 6.786899 \\
\hline $\mathrm{H}$ & 6.2521050 & -4.0773019 & 6.2562401 \\
\hline $\mathrm{C}$ & 6.8345640 & -2.2058950 & 7.064786 \\
\hline $\mathrm{C}$ & 5.5618150 & -1.5405050 & 6.5998100 \\
\hline $\mathrm{H}$ & 5.7918871 & -0.8656717 & 5.755137. \\
\hline $\mathrm{H}$ & 4.8167715 & -2.2754219 & 6.268968 \\
\hline $\mathrm{H}$ & 5.1463366 & -0.9231951 & $7.399030^{\circ}$ \\
\hline $\mathrm{C}$ & 7.1775290 & -2.6359090 & 12.171440 \\
\hline $\mathrm{H}$ & 7.1989687 & -3.7221586 & $11.975076^{\circ}$ \\
\hline $\mathrm{H}$ & 7.5385927 & -2.4304827 & 13.180421 \\
\hline $\mathrm{H}$ & 6.1221936 & -2.3063320 & 12.122035 \\
\hline $\mathrm{C}$ & 7.9845430 & -1.9177100 & 11.124679 \\
\hline $\mathrm{C}$ & 8.3698340 & -0.6005100 & 11.336325 \\
\hline $\mathrm{H}$ & 8.0627144 & -0.1522050 & 12.280637 \\
\hline $\mathrm{C}$ & 9.0590380 & 0.1923750 & 10.4372330 \\
\hline $\mathrm{C}$ & 9.3591000 & 1.6272620 & 10.757125 \\
\hline $\mathrm{H}$ & 9.0753808 & 2.2696844 & 9.922353 \\
\hline $\mathrm{H}$ & 8.8598822 & 1.9611208 & 11.681799 \\
\hline $\mathrm{H}$ & 10.4433612 & 1.7357847 & 10.881240 \\
\hline $\mathrm{C}$ & 13.1408560 & -2.8297920 & 10.150945 \\
\hline $\mathrm{H}$ & 12.7295778 & -3.6661945 & 10.737150 \\
\hline $\mathrm{H}$ & 14.0773921 & -3.1446891 & 9.659032 \\
\hline $\mathrm{H}$ & 13.3629024 & -2.0053988 & 10.830333 \\
\hline $\mathrm{C}$ & 12.0920060 & -2.3771460 & 9.141182 \\
\hline $\mathrm{C}$ & 12.4885530 & -1.8143560 & 7.940776 \\
\hline $\mathrm{H}$ & 13.5570051 & -1.7344979 & 7.745535 \\
\hline $\mathrm{C}$ & 11.6197760 & -1.4039570 & 6.934082 \\
\hline $\mathrm{C}$ & 12.1419340 & -0.8788880 & 5.612666 \\
\hline $\mathrm{H}$ & 11.3946462 & -0.1906530 & 5.187015 \\
\hline $\mathrm{H}$ & 13.1067557 & -0.3662946 & 5.721268 \\
\hline $\mathrm{H}$ & 12.27 & -1.7177086 & 4.916107 \\
\hline
\end{tabular}




\begin{tabular}{|c|c|c|c|}
\hline $\mathrm{Ga}$ & 9.3092320 & 5.5400100 & 8.5530960 \\
\hline 0 & 9.1669420 & 3.7486630 & 7.7583720 \\
\hline O & 7.6496870 & 6.0903520 & 7.664020 \\
\hline O & 8.2417020 & 4.9489300 & 10.087935 \\
\hline $\mathrm{O}$ & 9.4857900 & 7.3358830 & 9.310983 \\
\hline O & 10.9096650 & 4.9987210 & 9.509382 \\
\hline O & 10.3636120 & 6.1205280 & 7.012925 \\
\hline C & 8.3683040 & 1.8603750 & 6.625660 \\
\hline $\mathrm{H}$ & 8.6780098 & 1.2130603 & 7.468388 \\
\hline $\mathrm{H}$ & 7.4719986 & 1.4549384 & 6.161130 \\
\hline $\mathrm{H}$ & 9.2046618 & 1.8333131 & 5.903511 \\
\hline c & 8.1980410 & 3.2869640 & 7.098068 \\
\hline C & 7.0437550 & 3.9976190 & 6.786899 \\
\hline $\mathrm{H}$ & 6.2609916 & 3.4657876 & 6.255001 \\
\hline C & 6.8345640 & 5.3382050 & 7.064786 \\
\hline C & 5.5618150 & 6.0035950 & 6.599810 \\
\hline $\mathrm{H}$ & 5.8103864 & 6.6791016 & 5.759429 \\
\hline $\mathrm{H}$ & 4.8271577 & 5.2703975 & 6.262283 \\
\hline $\mathrm{H}$ & 5.1458709 & 6.6255835 & 7.392849 \\
\hline C & 7.1775290 & 4.9081910 & 12.171440 \\
\hline $\mathrm{H}$ & 7.1911368 & 3.8350834 & 11.969375 \\
\hline H & 7.5370267 & 5.1226083 & 13.178553 \\
\hline $\mathrm{H}$ & 6.1211227 & 5.2529803 & 12.120082 \\
\hline C & 7.9845430 & 5.6263900 & 11.124679 \\
\hline C & 8.3698340 & 6.9435900 & 11.336325 \\
\hline $\mathrm{H}$ & 8.0564098 & 7.4090556 & 12.271385 \\
\hline c & 9.0590380 & 7.7364750 & 10.437233 \\
\hline C & 9.3591000 & 9.1713620 & 10.757125 \\
\hline $\mathrm{H}$ & 9.0563938 & 9.7986658 & 9.890881 \\
\hline $\mathrm{H}$ & 8.8646803 & 9.5283619 & 11.662414 \\
\hline $\mathrm{H}$ & 10.4473739 & 9.2957010 & 10.855046 \\
\hline $\mathrm{C}$ & 13.1408560 & 4.7143080 & 10.150945 \\
\hline $\mathrm{H}$ & 12.7324221 & 3.9198682 & 10.774070 \\
\hline $\mathrm{H}$ & 14.0788499 & 4.4092706 & 9.68083 \\
\hline $\mathrm{H}$ & 13.3765306 & 5.5787706 & 10.82867 \\
\hline $\mathrm{C}$ & 12.0920060 & 5.1669540 & 9.14118 \\
\hline $\mathrm{C}$ & 12.4885530 & 5.7297440 & 7.940776 \\
\hline $\mathrm{H}$ & 13.5634237 & 5.8126708 & 7.753084 \\
\hline $\mathrm{C}$ & 11.6197760 & 6.1401430 & 6.934082 \\
\hline $\mathrm{C}$ & 12.1419340 & 6.6652120 & 5.612666 \\
\hline $\mathrm{H}$ & 11.3943149 & 7.3285959 & 5.176136 \\
\hline 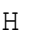 & 13.1073019 & 7.1744062 & 5.718278 \\
\hline & 12.2900517 & 5.8081198 & 4.923225 \\
\hline
\end{tabular}

\section{Compound 2a - X-ray cluster}

$\begin{array}{lrrr}261 & & & \\ \mathrm{Cu} & 5.135700 & 0.000000 & 0.000000 \\ \mathrm{O} & 3.470447 & 0.956207 & -0.065317 \\ \mathrm{O} & 5.870571 & 0.935842 & -1.511206 \\ \mathrm{C} & 3.214688 & 1.942498 & -0.820244 \\ \mathrm{C} & 4.070655 & 2.458090 & -1.805867 \\ \mathrm{H} & 3.778107 & 3.213892 & -2.301330 \\ \mathrm{C} & 5.336837 & 1.921670 & -2.102558 \\ \mathrm{C} & 1.885546 & 2.616378 & -0.595187 \\ \mathrm{H} & 1.250971 & 1.970267 & -0.219980 \\ \mathrm{H} & 1.542467 & 2.954244 & -1.448484 \\ \mathrm{H} & 1.999648 & 3.362460 & 0.029331 \\ \mathrm{C} & 6.150126 & 2.557136 & -3.200428 \\ \mathrm{H} & 6.805160 & 1.911025 & -3.536602 \\ \mathrm{H} & 6.617257 & 3.343021 & -2.847331 \\ \mathrm{H} & 5.556114 & 2.830668 & -3.930310 \\ \mathrm{O} & 6.800953 & -0.956207 & 0.065317 \\ \mathrm{O} & 4.400829 & -0.935842 & 1.511206 \\ \mathrm{C} & 7.056712 & -1.942498 & 0.820244 \\ \mathrm{C} & 6.200745 & -2.458090 & 1.805867 \\ \mathrm{H} & 6.493293 & -3.213892 & 2.301330 \\ \mathrm{C} & 4.934563 & -1.921670 & 2.102558 \\ \mathrm{C} & 8.385854 & -2.616378 & 0.595187\end{array}$




\begin{tabular}{|c|c|c|c|}
\hline $\mathrm{H}$ & 9.020429 & -1.970267 & 0.219980 \\
\hline $\mathrm{H}$ & 8.728933 & -2.954244 & 1.448484 \\
\hline $\mathrm{H}$ & 8.271752 & -3.362460 & -0.029331 \\
\hline C & 4.121274 & -2.557136 & 3.200428 \\
\hline $\mathrm{H}$ & 3.466240 & -1.911025 & 3.536602 \\
\hline $\mathrm{H}$ & 3.654143 & -3.343021 & 2.847331 \\
\hline $\mathrm{H}$ & 4.715286 & -2.830668 & 3.930310 \\
\hline $\mathrm{Cu}$ & 5.135700 & -4.628300 & 0.000000 \\
\hline 0 & 3.470447 & -3.672093 & -0.065317 \\
\hline 0 & 5.870571 & -3.692458 & -1.511206 \\
\hline $\mathrm{C}$ & 3.214688 & -2.685802 & -0.820244 \\
\hline $\mathrm{C}$ & 4.070655 & -2.170210 & -1.805867 \\
\hline $\mathrm{H}$ & 3.778107 & -1.414408 & -2.301330 \\
\hline $\mathrm{C}$ & 5.336837 & -2.706630 & -2.102558 \\
\hline $\mathrm{C}$ & 1.885546 & -2.011922 & -0.595187 \\
\hline $\mathrm{H}$ & 1.250971 & -2.658033 & -0.219980 \\
\hline $\mathrm{H}$ & 1.542467 & -1.674056 & -1.448484 \\
\hline $\mathrm{H}$ & 1.999648 & -1.265840 & 0.029331 \\
\hline $\mathrm{C}$ & 6.150126 & -2.071164 & -3.200428 \\
\hline $\mathrm{H}$ & 6.805160 & -2.717275 & -3.536602 \\
\hline $\mathrm{H}$ & 6.617257 & -1.285279 & -2.847331 \\
\hline $\mathrm{H}$ & 5.556114 & -1.797632 & -3.930310 \\
\hline 0 & 6.800953 & -5.584507 & 0.065317 \\
\hline 0 & 4.400829 & -5.564142 & 1.511206 \\
\hline $\mathrm{C}$ & 7.056712 & -6.570798 & 0.820244 \\
\hline $\mathrm{C}$ & 6.200745 & -7.086390 & 1.805867 \\
\hline $\mathrm{H}$ & 6.493293 & -7.842192 & 2.301330 \\
\hline $\mathrm{C}$ & 4.934563 & -6.549970 & 2.102558 \\
\hline $\mathrm{C}$ & 8.385854 & -7.244678 & 0.595187 \\
\hline $\mathrm{H}$ & 9.020429 & -6.598567 & 0.219980 \\
\hline $\mathrm{H}$ & 8.728933 & -7.582544 & 1.448484 \\
\hline $\mathrm{H}$ & 8.271752 & -7.990760 & -0.029331 \\
\hline $\mathrm{C}$ & 4.121274 & -7.185436 & 3.200428 \\
\hline $\mathrm{H}$ & 3.466240 & -6.539325 & 3.536602 \\
\hline $\mathrm{H}$ & 3.654143 & -7.971321 & 2.847331 \\
\hline $\mathrm{H}$ & 4.715286 & -7.458968 & 3.930310 \\
\hline $\mathrm{Cu}$ & 5.135700 & 4.628300 & 0.000000 \\
\hline 0 & 3.470447 & 5.584507 & -0.065317 \\
\hline 0 & 5.870571 & 5.564142 & -1.511206 \\
\hline $\mathrm{C}$ & 3.214688 & 6.570798 & -0.820244 \\
\hline $\mathrm{C}$ & 4.070655 & 7.086390 & -1.805867 \\
\hline $\mathrm{H}$ & 3.778107 & 7.842192 & -2.301330 \\
\hline $\mathrm{C}$ & 5.336837 & 6.549970 & -2.102558 \\
\hline $\mathrm{C}$ & 1.885546 & 7.244678 & -0.595187 \\
\hline $\mathrm{H}$ & 1.250971 & 6.598567 & -0.219980 \\
\hline $\mathrm{H}$ & 1.542467 & 7.582544 & -1.448484 \\
\hline $\mathrm{H}$ & 1.999648 & 7.990760 & 0.029331 \\
\hline $\mathrm{C}$ & 6.150126 & 7.185436 & -3.200428 \\
\hline $\mathrm{H}$ & 6.805160 & 6.539325 & -3.536602 \\
\hline $\mathrm{H}$ & 6.617257 & 7.971321 & -2.847331 \\
\hline $\mathrm{H}$ & 5.556114 & 7.458968 & -3.930310 \\
\hline 0 & 6.800953 & 3.672093 & 0.065317 \\
\hline 0 & 4.400829 & 3.692458 & 1.511206 \\
\hline $\mathrm{C}$ & 7.056712 & 2.685802 & 0.820244 \\
\hline $\mathrm{C}$ & 6.200745 & 2.170210 & 1.805867 \\
\hline $\mathrm{H}$ & 6.493293 & 1.414408 & 2.301330 \\
\hline $\mathrm{C}$ & 4.934563 & 2.706630 & 2.102558 \\
\hline $\mathrm{C}$ & 8.385854 & 2.011922 & 0.595187 \\
\hline $\mathrm{H}$ & 9.020429 & 2.658033 & 0.219980 \\
\hline $\mathrm{H}$ & 8.728933 & 1.674056 & 1.448484 \\
\hline $\mathrm{H}$ & 8.271752 & 1.265840 & -0.029331 \\
\hline $\mathrm{C}$ & 4.121274 & 2.071164 & 3.200428 \\
\hline $\mathrm{H}$ & 3.466240 & 2.717275 & 3.536602 \\
\hline $\mathrm{H}$ & 3.654143 & 1.285279 & 2.847331 \\
\hline $\mathrm{H}$ & 4.715286 & 1.797632 & 3.930310 \\
\hline $\mathrm{Cu}$ & 0.234830 & -2.314150 & -5.640514 \\
\hline 0 & 1.900083 & -1.357943 & -5.575197 \\
\hline 0 & -0.500041 & -1.378308 & -4.129307 \\
\hline $\mathrm{C}$ & 2.155842 & -0.371652 & -4.82027 \\
\hline $\mathrm{C}$ & 1.299875 & 0.143940 & -3.83464 \\
\hline
\end{tabular}




\begin{tabular}{|c|c|c|c|}
\hline $\mathrm{H}$ & 1.592422 & 0.899742 & -3.339184 \\
\hline $\mathrm{C}$ & 0.033693 & -0.392480 & -3.537956 \\
\hline $\mathrm{C}$ & 3.484984 & 0.302228 & -5.045327 \\
\hline $\mathrm{H}$ & 4.119559 & -0.343883 & -5.420534 \\
\hline $\mathrm{H}$ & 3.828062 & 0.640094 & -4.192030 \\
\hline $\mathrm{H}$ & 3.370882 & 1.048310 & -5.669844 \\
\hline $\mathrm{C}$ & -0.779596 & 0.242986 & -2.440086 \\
\hline $\mathrm{H}$ & -1.434630 & -0.403125 & -2.103912 \\
\hline $\mathrm{H}$ & -1.246727 & 1.028871 & -2.793182 \\
\hline $\mathrm{H}$ & -0.185585 & 0.516518 & -1.710204 \\
\hline 0 & -1.430424 & -3.270357 & -5.705831 \\
\hline 0 & 0.969700 & -3.249992 & -7.151720 \\
\hline $\mathrm{C}$ & -1.686182 & -4.256648 & -6.460757 \\
\hline $\mathrm{C}$ & -0.830215 & -4.772240 & -7.446381 \\
\hline $\mathrm{H}$ & -1.122763 & -5.528042 & -7.941843 \\
\hline $\mathrm{C}$ & 0.435966 & -4.235820 & -7.743072 \\
\hline $\mathrm{C}$ & -3.015324 & -4.930528 & -6.235701 \\
\hline $\mathrm{H}$ & -3.649900 & -4.284417 & -5.860494 \\
\hline $\mathrm{H}$ & -3.358403 & -5.268394 & -7.088998 \\
\hline $\mathrm{H}$ & -2.901223 & -5.676610 & -5.611183 \\
\hline $\mathrm{C}$ & 1.249255 & -4.871286 & -8.840941 \\
\hline $\mathrm{H}$ & 1.904289 & -4.225175 & -9.177116 \\
\hline $\mathrm{H}$ & 1.716387 & -5.657171 & -8.487845 \\
\hline $\mathrm{H}$ & 0.655244 & -5.144818 & -9.570824 \\
\hline $\mathrm{Cu}$ & 10.036570 & 2.314150 & 5.640514 \\
\hline 0 & 11.701824 & 3.270357 & 5.705831 \\
\hline 0 & 9.301700 & 3.249992 & 7.151720 \\
\hline $\mathrm{C}$ & 11.957582 & 4.256648 & 6.460757 \\
\hline $\mathrm{C}$ & 11.101615 & 4.772240 & 7.446381 \\
\hline $\mathrm{H}$ & 11.394163 & 5.528042 & 7.941843 \\
\hline $\mathrm{C}$ & 9.835434 & 4.235820 & 7.743072 \\
\hline $\mathrm{C}$ & 13.286724 & 4.930528 & 6.235701 \\
\hline $\mathrm{H}$ & 13.921300 & 4.284417 & 5.860494 \\
\hline $\mathrm{H}$ & 13.629803 & 5.268394 & 7.088998 \\
\hline $\mathrm{H}$ & 13.172623 & 5.676610 & 5.611183 \\
\hline $\mathrm{C}$ & 9.022145 & 4.871286 & 8.840941 \\
\hline $\mathrm{H}$ & 8.367111 & 4.225175 & 9.17711 \\
\hline $\mathrm{H}$ & 8.555013 & 5.657171 & 8.48784 \\
\hline $\mathrm{H}$ & 9.616156 & 5.144818 & 9.570824 \\
\hline 0 & 8.371317 & 1.357943 & 5.575197 \\
\hline 0 & 10.771441 & 1.378308 & 4.129307 \\
\hline $\mathrm{C}$ & 8.115558 & 0.371652 & 4.820270 \\
\hline $\mathrm{C}$ & 8.971525 & -0.143940 & 3.834647 \\
\hline $\mathrm{H}$ & 8.678978 & -0.899742 & 3.339184 \\
\hline $\mathrm{C}$ & 10.237707 & 0.392480 & 3.537956 \\
\hline $\mathrm{C}$ & 6.786416 & -0.302228 & 5.045327 \\
\hline $\mathrm{H}$ & 6.151841 & 0.343883 & 5.420534 \\
\hline $\mathrm{H}$ & 6.443338 & -0.640094 & 4.192030 \\
\hline $\mathrm{H}$ & 6.900518 & -1.048310 & 5.66984 \\
\hline $\mathrm{C}$ & 11.050996 & -0.242986 & 2.44008 \\
\hline $\mathrm{H}$ & 11.706030 & 0.403125 & 2.103912 \\
\hline $\mathrm{H}$ & 11.518127 & -1.028871 & 2.793182 \\
\hline $\mathrm{H}$ & 10.456985 & -0.516518 & 1.710204 \\
\hline $\mathrm{Cu}$ & -0.234830 & 2.314150 & 5.640514 \\
\hline 0 & 1.430424 & 3.270357 & 5.705831 \\
\hline 0 & -0.969700 & 3.249992 & 7.15172 \\
\hline $\mathrm{C}$ & 1.686182 & 4.256648 & 6.460757 \\
\hline $\mathrm{C}$ & 0.830215 & 4.772240 & 7.446381 \\
\hline $\mathrm{H}$ & 1.122763 & 5.528042 & 7.94184 \\
\hline $\mathrm{C}$ & -0.435966 & 4.235820 & 7.743072 \\
\hline $\mathrm{C}$ & 3.015324 & 4.930528 & 6.23570 \\
\hline $\mathrm{H}$ & 3.649900 & 4.284417 & 5.86049 \\
\hline $\mathrm{H}$ & 3.358403 & 5.268394 & 7.08899 \\
\hline $\mathrm{H}$ & 2.901223 & 5.676610 & 5.611183 \\
\hline $\mathrm{C}$ & -1.249255 & 4.871286 & 8.840941 \\
\hline $\mathrm{H}$ & -1.904289 & 4.225175 & 9.17711 \\
\hline $\mathrm{H}$ & -1.716387 & 5.657171 & 8.48784 \\
\hline $\mathrm{H}$ & -0.655244 & 5.144818 & 9.570824 \\
\hline 0 & -1.900083 & 1.357943 & 5.57519 \\
\hline 0 & 0.500041 & 1.378308 & 4.12930 \\
\hline
\end{tabular}




\begin{tabular}{|c|c|c|c|}
\hline $\mathrm{C}$ & -2.155842 & 0.371652 & 4.820270 \\
\hline $\mathrm{C}$ & -1.299875 & -0.143940 & 3.834647 \\
\hline $\mathrm{H}$ & -1.592422 & -0.899742 & 3.339184 \\
\hline C & -0.033693 & 0.392480 & 3.537956 \\
\hline $\mathrm{C}$ & -3.484984 & -0.302228 & 5.045327 \\
\hline $\mathrm{H}$ & -4.119559 & 0.343883 & 5.420534 \\
\hline $\mathrm{H}$ & -3.828062 & -0.640094 & 4.192030 \\
\hline $\mathrm{H}$ & -3.370882 & -1.048310 & 5.669844 \\
\hline $\mathrm{C}$ & 0.779596 & -0.242986 & 2.440086 \\
\hline $\mathrm{H}$ & 1.434630 & 0.403125 & 2.103912 \\
\hline $\mathrm{H}$ & 1.246727 & -1.028871 & 2.793182 \\
\hline $\mathrm{H}$ & 0.185585 & -0.516518 & 1.710204 \\
\hline $\mathrm{Cu}$ & 10.506230 & -2.314150 & -5.640514 \\
\hline 0 & 12.171483 & -1.357943 & -5.575197 \\
\hline 0 & 9.771359 & -1.378308 & -4.129307 \\
\hline $\mathrm{C}$ & 12.427242 & -0.371652 & -4.820270 \\
\hline $\mathrm{C}$ & 11.571275 & 0.143940 & -3.834647 \\
\hline $\mathrm{H}$ & 11.863822 & 0.899742 & -3.339184 \\
\hline $\mathrm{C}$ & 10.305093 & -0.392480 & -3.537956 \\
\hline $\mathrm{C}$ & 13.756384 & 0.302228 & -5.045327 \\
\hline $\mathrm{H}$ & 14.390959 & -0.343883 & -5.420534 \\
\hline $\mathrm{H}$ & 14.099462 & 0.640094 & -4.192030 \\
\hline $\mathrm{H}$ & 13.642282 & 1.048310 & -5.669844 \\
\hline $\mathrm{C}$ & 9.491804 & 0.242986 & -2.440086 \\
\hline $\mathrm{H}$ & 8.836770 & -0.403125 & -2.103912 \\
\hline $\mathrm{H}$ & 9.024673 & 1.028871 & -2.793182 \\
\hline $\mathrm{H}$ & 10.085815 & 0.516518 & -1.710204 \\
\hline O & 8.840976 & -3.270357 & -5.705831 \\
\hline 0 & 11.241100 & -3.249992 & -7.151720 \\
\hline $\mathrm{C}$ & 8.585218 & -4.256648 & -6.460757 \\
\hline $\mathrm{C}$ & 9.441185 & -4.772240 & -7.446381 \\
\hline $\mathrm{H}$ & 9.148637 & -5.528042 & -7.941843 \\
\hline $\mathrm{C}$ & 10.707366 & -4.235820 & -7.743072 \\
\hline $\mathrm{C}$ & 7.256076 & -4.930528 & -6.235701 \\
\hline $\mathrm{H}$ & 6.621500 & -4.284417 & -5.860494 \\
\hline $\mathrm{H}$ & 6.912997 & -5.268394 & -7.088998 \\
\hline $\mathrm{H}$ & 7.370177 & -5.676610 & -5.611183 \\
\hline $\mathrm{C}$ & 11.520655 & -4.871286 & -8.840941 \\
\hline $\mathrm{H}$ & 12.175689 & -4.225175 & -9.177116 \\
\hline $\mathrm{H}$ & 11.987787 & -5.657171 & -8.48784 \\
\hline $\mathrm{H}$ & 10.926644 & -5.144818 & -9.570824 \\
\hline $\mathrm{Cu}$ & 0.234830 & 2.314150 & -5.640514 \\
\hline 0 & 1.900083 & 3.270357 & -5.575197 \\
\hline 0 & -0.500041 & 3.249992 & -4.129307 \\
\hline $\mathrm{C}$ & 2.155842 & 4.256648 & -4.820270 \\
\hline $\mathrm{C}$ & 1.299875 & 4.772240 & -3.834647 \\
\hline $\mathrm{H}$ & 1.592422 & 5.528042 & -3.339184 \\
\hline $\mathrm{C}$ & 0.033693 & 4.235820 & -3.537956 \\
\hline $\mathrm{C}$ & 3.484984 & 4.930528 & -5.045327 \\
\hline $\mathrm{H}$ & 4.119559 & 4.284417 & -5.420534 \\
\hline $\mathrm{H}$ & 3.828062 & 5.268394 & -4.192030 \\
\hline $\mathrm{H}$ & 3.370882 & 5.676610 & -5.669844 \\
\hline $\mathrm{C}$ & -0.779596 & 4.871286 & -2.440086 \\
\hline $\mathrm{H}$ & -1.434630 & 4.225175 & -2.103912 \\
\hline $\mathrm{H}$ & -1.246727 & 5.657171 & -2.793182 \\
\hline $\mathrm{H}$ & -0.185585 & 5.144818 & -1.710204 \\
\hline 0 & -1.430424 & 1.357943 & -5.705831 \\
\hline 0 & 0.969700 & 1.378308 & -7.151720 \\
\hline $\mathrm{C}$ & -1.686182 & 0.371652 & -6.460757 \\
\hline $\mathrm{C}$ & -0.830215 & -0.143940 & -7.446381 \\
\hline $\mathrm{H}$ & -1.122763 & -0.899742 & -7.941843 \\
\hline $\mathrm{C}$ & 0.435966 & 0.392480 & -7.743072 \\
\hline $\mathrm{C}$ & -3.015324 & -0.302228 & -6.235701 \\
\hline $\mathrm{H}$ & -3.649900 & 0.343883 & -5.86049 \\
\hline $\mathrm{H}$ & -3.358403 & -0.640094 & -7.08899 \\
\hline $\mathrm{H}$ & -2.901223 & -1.048310 & -5.611183 \\
\hline $\mathrm{C}$ & 1.249255 & -0.242986 & -8.84094 \\
\hline $\mathrm{H}$ & 1.904289 & 0.403125 & -9.177116 \\
\hline $\mathrm{H}$ & 1.716387 & -1.028871 & -8.48784 \\
\hline $\mathrm{H}$ & 0.655244 & -0.516518 & -9.57082 \\
\hline
\end{tabular}




$\begin{array}{lrrr}\mathrm{Cu} & 10.036570 & -2.314150 & 5.640514 \\ \mathrm{O} & 11.701824 & -1.357943 & 5.705831 \\ \mathrm{O} & 9.301700 & -1.378308 & 7.151720 \\ \mathrm{C} & 11.957582 & -0.371652 & 6.460757 \\ \mathrm{C} & 11.101615 & 0.143940 & 7.446381 \\ \mathrm{H} & 11.394163 & 0.899742 & 7.941843 \\ \mathrm{C} & 9.835434 & -0.392480 & 7.743072 \\ \mathrm{C} & 13.286724 & 0.302228 & 6.235701 \\ \mathrm{H} & 13.921300 & -0.343883 & 5.860494 \\ \mathrm{H} & 13.629803 & 0.640094 & 7.088998 \\ \mathrm{H} & 13.172623 & 1.048310 & 5.611183 \\ \mathrm{C} & 9.022145 & 0.242986 & 8.840941 \\ \mathrm{H} & 8.367111 & -0.403125 & 9.177116 \\ \mathrm{H} & 8.555013 & 1.028871 & 8.487845 \\ \mathrm{H} & 9.616156 & 0.516518 & 9.570824 \\ \mathrm{O} & 8.371317 & -3.270357 & 5.575197 \\ \mathrm{O} & 10.771441 & -3.249992 & 4.129307 \\ \mathrm{C} & 8.115558 & -4.256648 & 4.820270 \\ \mathrm{C} & 8.971525 & -4.772240 & 3.834647 \\ \mathrm{H} & 8.678978 & -5.528042 & 3.339184 \\ \mathrm{C} & 10.237707 & -4.235820 & 3.537956 \\ \mathrm{C} & 6.786416 & -4.930528 & 5.045327 \\ \mathrm{H} & 6.151841 & -4.284417 & 5.420534 \\ \mathrm{H} & 6.443338 & -5.268394 & 4.192030 \\ \mathrm{H} & 6.900518 & -5.676610 & 5.669844 \\ \mathrm{C} & 11.050996 & -4.871286 & 2.440086 \\ \mathrm{H} & 11.706030 & -4.225175 & 2.103912 \\ \mathrm{H} & 11.518127 & -5.657171 & 2.793182 \\ \mathrm{H} & 10.456985 & -5.144818 & 1.710204\end{array}$

\section{Compound 2a with Ca atoms in embedding - optimized central molecule}

$\begin{array}{lrrr}261 & & \\ \mathrm{Cu} & 5.1323123 & 0.0003672 & 0.0015527 \\ \mathrm{O} & 3.4497759 & 0.9541777 & -0.0807462 \\ \mathrm{O} & 5.8506792 & 0.9509925 & -1.5153522 \\ \mathrm{C} & 3.2049363 & 1.9585903 & -0.8004195 \\ \mathrm{C} & 4.0731402 & 2.4921725 & -1.7679058 \\ \mathrm{H} & 3.7161805 & 3.3271685 & -2.3565304 \\ \mathrm{C} & 5.3323773 & 1.9549953 & -2.0750507 \\ \mathrm{C} & 1.8992898 & 2.6461809 & -0.5522562 \\ \mathrm{H} & 1.1431416 & 1.9134439 & -0.2754031 \\ \mathrm{H} & 1.5785249 & 3.2284545 & -1.4142961 \\ \mathrm{H} & 2.0430798 & 3.3300242 & 0.2895719 \\ \mathrm{C} & 6.1750279 & 2.6157053 & -3.1204489 \\ \mathrm{H} & 6.5981529 & 1.8573716 & -3.7799265 \\ \mathrm{H} & 6.9928935 & 3.1284837 & -2.6081810 \\ \mathrm{H} & 5.6179515 & 3.3485722 & -3.6969508 \\ \mathrm{O} & 6.8151536 & -0.9524616 & 0.0847008 \\ \mathrm{O} & 4.4134650 & -0.9508554 & 1.5180508 \\ \mathrm{C} & 7.0606217 & -1.9559784 & 0.8054184 \\ \mathrm{C} & 6.1925098 & -2.4898704 & 1.7727426 \\ \mathrm{H} & 6.5499988 & -3.3241047 & 2.3621058 \\ \mathrm{C} & 4.9325429 & -1.9537870 & 2.0789107 \\ \mathrm{C} & 8.3675219 & -2.6417875 & 0.5588306 \\ \mathrm{H} & 9.1230339 & -1.9073723 & 0.2846788 \\ \mathrm{H} & 8.6874555 & -3.2248424 & 1.4206650 \\ \mathrm{H} & 8.2264901 & -3.3244281 & -0.2844391 \\ \mathrm{C} & 4.0903338 & -2.6143244 & 3.1248129 \\ \mathrm{H} & 3.6661228 & -1.8559657 & 3.7836442 \\ \mathrm{H} & 3.2732222 & -3.1285904 & 2.6128227 \\ \mathrm{H} & 4.6481415 & -3.3459531 & 3.7022219 \\ \mathrm{Ca} & 5.1357000 & -4.6283000 & 0.0000000 \\ \mathrm{O} & 3.4704470 & -3.6720930 & -0.0653170 \\ \mathrm{O} & 5.8705710 & -3.6924580 & -1.5112060 \\ \mathrm{C} & 3.2146880 & -2.6858020 & -0.8202440 \\ \mathrm{C} & 4.0706550 & -2.1702100 & -1.8058670 \\ \mathrm{H} & 3.7080659 & -1.3409284 & -2.4124282 \\ \mathrm{C} & 5.3368370 & -2.7066300 & -2.1025580 \\ \mathrm{C} & 1.8855460 & -2.0119220 & -0.5951870\end{array}$




\begin{tabular}{|c|c|c|c|}
\hline $\mathrm{H}$ & 1.1251742 & -2.7708814 & -0.3671478 \\
\hline $\mathrm{H}$ & 1.5742327 & -1.3875334 & -1.4418266 \\
\hline $\mathrm{H}$ & 1.9979662 & -1.3599992 & 0.2845380 \\
\hline C & 6.1501260 & -2.0711640 & -3.2004280 \\
\hline $\mathrm{H}$ & 6.6046878 & -2.8449256 & -3.8348038 \\
\hline $\mathrm{H}$ & 6.9862230 & -1.5285117 & -2.7326445 \\
\hline $\mathrm{H}$ & 5.5755938 & -1.3617457 & -3.8065638 \\
\hline 0 & 6.8009530 & -5.5845070 & 0.0653170 \\
\hline 0 & 4.4008290 & -5.5641420 & 1.5112060 \\
\hline $\mathrm{C}$ & 7.0567120 & -6.5707980 & 0.8202440 \\
\hline $\mathrm{C}$ & 6.2007450 & -7.0863900 & 1.8058670 \\
\hline $\mathrm{H}$ & 6.5411688 & -7.9466137 & 2.3818398 \\
\hline $\mathrm{C}$ & 4.9345630 & -6.5499700 & 2.1025580 \\
\hline $\mathrm{C}$ & 8.3858540 & -7.2446780 & 0.5951870 \\
\hline $\mathrm{H}$ & 9.1664602 & -6.4735374 & 0.5250632 \\
\hline $\mathrm{H}$ & 8.6463541 & -7.9794268 & 1.3680194 \\
\hline $\mathrm{H}$ & 8.3592340 & -7.7497387 & -0.3837166 \\
\hline $\mathrm{C}$ & 4.1212740 & -7.1854360 & 3.2004280 \\
\hline $\mathrm{H}$ & 3.8965267 & -6.4203893 & 3.9601614 \\
\hline $\mathrm{H}$ & 3.1539129 & -7.5043455 & 2.7830730 \\
\hline $\mathrm{H}$ & 4.6163968 & -8.0406070 & 3.6776035 \\
\hline $\mathrm{Ca}$ & 5.1357000 & 4.6283000 & 0.0000000 \\
\hline 0 & 3.4704470 & 5.5845070 & -0.0653170 \\
\hline 0 & 5.8705710 & 5.5641420 & -1.5112060 \\
\hline $\mathrm{C}$ & 3.2146880 & 6.5707980 & -0.8202440 \\
\hline $\mathrm{C}$ & 4.0706550 & 7.0863900 & -1.8058670 \\
\hline $\mathrm{H}$ & 3.7302264 & 7.9466701 & -2.3817675 \\
\hline C & 5.3368370 & 6.5499700 & -2.1025580 \\
\hline $\mathrm{C}$ & 1.8855460 & 7.2446780 & -0.5951870 \\
\hline $\mathrm{H}$ & 1.1048984 & 6.4736414 & -0.5245778 \\
\hline $\mathrm{H}$ & 1.6248720 & 7.9792126 & -1.3681689 \\
\hline $\mathrm{H}$ & 1.9123777 & 7.7501266 & 0.3835233 \\
\hline $\mathrm{C}$ & 6.1501260 & 7.1854360 & -3.2004280 \\
\hline $\mathrm{H}$ & 6.3752274 & 6.4203218 & -3.9599602 \\
\hline $\mathrm{H}$ & 7.1172987 & 7.5047192 & -2.7829004 \\
\hline $\mathrm{H}$ & 5.6548539 & 8.0404214 & -3.6777875 \\
\hline 0 & 6.8009530 & 3.6720930 & 0.0653170 \\
\hline 0 & 4.4008290 & 3.6924580 & 1.5112060 \\
\hline $\mathrm{C}$ & 7.0567120 & 2.6858020 & 0.8202440 \\
\hline $\mathrm{C}$ & 6.2007450 & 2.1702100 & 1.8058670 \\
\hline $\mathrm{H}$ & 6.5633421 & 1.3410011 & 2.4124995 \\
\hline $\mathrm{C}$ & 4.9345630 & 2.7066300 & 2.1025580 \\
\hline $\mathrm{C}$ & 8.3858540 & 2.0119220 & 0.5951870 \\
\hline $\mathrm{H}$ & 9.1460539 & 2.7708242 & 0.3664226 \\
\hline $\mathrm{H}$ & 8.6974140 & 1.3880930 & 1.4421552 \\
\hline $\mathrm{H}$ & 8.2731331 & 1.3594056 & -0.2840063 \\
\hline $\mathrm{C}$ & 4.1212740 & 2.0711640 & 3.2004280 \\
\hline $\mathrm{H}$ & 3.6677648 & 2.8450600 & 3.8354467 \\
\hline $\mathrm{H}$ & 3.2843180 & 1.5296152 & 2.7329313 \\
\hline $\mathrm{H}$ & 4.6954353 & 1.3610166 & 3.8060327 \\
\hline $\mathrm{Ca}$ & 0.2348300 & -2.3141500 & -5.6405140 \\
\hline 0 & 1.9000830 & -1.3579430 & -5.5751970 \\
\hline 0 & -0.5000410 & -1.3783080 & -4.1293070 \\
\hline $\mathrm{C}$ & 2.1558420 & -0.3716520 & -4.8202700 \\
\hline $\mathrm{C}$ & 1.2998750 & 0.1439400 & -3.8346470 \\
\hline $\mathrm{H}$ & 1.6700082 & 0.9652119 & -3.2189279 \\
\hline $\mathrm{C}$ & 0.0336930 & -0.3924800 & -3.5379560 \\
\hline $\mathrm{C}$ & 3.4849840 & 0.3022280 & -5.0453270 \\
\hline $\mathrm{H}$ & 4.2238405 & -0.4517746 & -5.3443500 \\
\hline $\mathrm{H}$ & 3.8317565 & 0.8691460 & -4.1711481 \\
\hline $\mathrm{H}$ & 3.3568129 & 1.0099179 & -5.8817269 \\
\hline $\mathrm{C}$ & -0.7795960 & 0.2429860 & -2.4400860 \\
\hline $\mathrm{H}$ & -1.2363565 & -0.5470585 & -1.8279488 \\
\hline $\mathrm{H}$ & -1.5913975 & 0.8137332 & -2.9202678 \\
\hline $\mathrm{H}$ & -0.1977826 & 0.9301200 & -1.8144432 \\
\hline 0 & -1.4304240 & -3.2703570 & -5.7058310 \\
\hline 0 & 0.9697000 & -3.2499920 & -7.1517200 \\
\hline $\mathrm{C}$ & -1.6861820 & -4.2566480 & -6.4607570 \\
\hline $\mathrm{C}$ & -0.8302150 & -4.7722400 & -7.4463810 \\
\hline $\mathrm{H}$ & -1.1724531 & -5.6291738 & -8.025567 \\
\hline
\end{tabular}




\begin{tabular}{|c|c|c|c|}
\hline C & 0.4359660 & -4.2358200 & -7.7430720 \\
\hline$C$ & -3.0153240 & -4.9305280 & -6.2357010 \\
\hline I & -3.8147427 & -4.1802123 & -6.3377444 \\
\hline H & -3.2075267 & -5.7679193 & -6.9183061 \\
\hline $\mathrm{H}$ & -3.0541773 & -5.2885376 & -5.1951599 \\
\hline $\mathrm{C}$ & 1.2492550 & -4.8712860 & -8.8409410 \\
\hline $\mathrm{H}$ & 1.4833753 & -4.1032692 & -9.5945986 \\
\hline $\mathrm{H}$ & 2.2125009 & -5.2003346 & -8.4211860 \\
\hline $\mathrm{H}$ & 0.7497830 & -5.7201677 & -9.3244199 \\
\hline $\mathrm{Ca}$ & 10.0365700 & 2.3141500 & 5.6405140 \\
\hline 0 & 11.7018240 & 3.2703570 & 5.705831 \\
\hline O & 9.3017000 & 3.2499920 & 7.151720 \\
\hline C & 11.9575820 & 4.2566480 & 6.460757 \\
\hline $\mathrm{C}$ & 11.1016150 & 4.7722400 & 7.4463810 \\
\hline $\mathrm{H}$ & 11.4438068 & 5.6291784 & 8.0256197 \\
\hline $\mathrm{C}$ & 9.8354340 & 4.2358200 & 7.743072 \\
\hline $\mathrm{C}$ & 13.2867240 & 4.9305280 & 6.2357010 \\
\hline $\mathrm{H}$ & 14.0861226 & 4.1801476 & 6.337335 \\
\hline $\mathrm{H}$ & 13.4790623 & 5.7676470 & 6.918624 \\
\hline $\mathrm{H}$ & 13.3253527 & 5.2889968 & 5.195329 \\
\hline $\mathrm{C}$ & 9.0221450 & 4.8712860 & 8.840941 \\
\hline $\mathrm{H}$ & 8.7875258 & 4.1031981 & 9.594384 \\
\hline $\mathrm{H}$ & 8.0591054 & 5.2008554 & 8.4211018 \\
\hline $\mathrm{H}$ & 9.5217825 & 5.7198609 & 9.3248016 \\
\hline 0 & 8.3713170 & 1.3579430 & 5.5751970 \\
\hline 0 & 10.7714410 & 1.3783080 & 4.1293070 \\
\hline $\mathrm{C}$ & 8.1155580 & 0.3716520 & 4.8202700 \\
\hline $\mathrm{C}$ & 8.9715250 & -0.1439400 & 3.8346470 \\
\hline $\mathrm{H}$ & 8.6013713 & -0.9652760 & 3.2190569 \\
\hline $\mathrm{C}$ & 10.2377070 & 0.3924800 & 3.5379560 \\
\hline C & 6.7864160 & -0.3022280 & 5.0453270 \\
\hline $\mathrm{H}$ & 6.0483894 & 0.4514261 & 5.3473094 \\
\hline $\mathrm{H}$ & 6.4382397 & -0.8665816 & 4.1700298 \\
\hline $\mathrm{H}$ & 6.9151672 & -1.0123558 & 5.8795896 \\
\hline $\mathrm{C}$ & 11.0509960 & -0.2429860 & 2.4400860 \\
\hline $\mathrm{H}$ & 11.5047401 & 0.5472174 & 1.8258762 \\
\hline $\mathrm{H}$ & 11.8650333 & -0.8104467 & 2.9202912 \\
\hline $\mathrm{H}$ & 10.4702609 & -0.9328589 & 1.8165043 \\
\hline $\mathrm{Ca}$ & -0.2348300 & 2.3141500 & 5.640514 \\
\hline 0 & 1.4304240 & 3.2703570 & 5.705831 \\
\hline 0 & -0.9697000 & 3.2499920 & 7.151720 \\
\hline $\mathrm{C}$ & 1.6861820 & 4.2566480 & 6.460757 \\
\hline $\mathrm{C}$ & 0.8302150 & 4.7722400 & 7.446381 \\
\hline $\mathrm{H}$ & 1.1728103 & 5.6289968 & 8.0255887 \\
\hline C & -0.4359660 & 4.2358200 & 7.7430720 \\
\hline $\mathrm{C}$ & 3.0153240 & 4.9305280 & 6.2357010 \\
\hline $\mathrm{H}$ & 3.8173915 & 4.1871801 & 6.3655899 \\
\hline $\mathrm{H}$ & 3.1958088 & 5.7834972 & 6.902051 \\
\hline $\mathrm{H}$ & 3.0659077 & 5.2641189 & 5.1872850 \\
\hline C & -1.2492550 & 4.8712860 & 8.8409410 \\
\hline $\mathrm{H}$ & -1.4758720 & 4.1055969 & 9.5989809 \\
\hline $\mathrm{H}$ & -2.2158919 & 5.1928653 & 8.4234244 \\
\hline $\mathrm{H}$ & -0.7523973 & 5.7250904 & 9.3186027 \\
\hline O & -1.9000830 & 1.3579430 & 5.5751970 \\
\hline O & 0.5000410 & 1.3783080 & 4.1293070 \\
\hline C & -2.1558420 & 0.3716520 & 4.8202700 \\
\hline C & -1.2998750 & -0.1439400 & $3.834647 C$ \\
\hline $\mathrm{H}$ & -1.6416505 & -0.9993823 & 3.2531732 \\
\hline C & -0.0336930 & 0.3924800 & 3.5379560 \\
\hline C & -3.4849840 & -0.3022280 & 5.0453270 \\
\hline $\mathrm{H}$ & -4.2851904 & 0.4462745 & 4.9383136 \\
\hline $\mathrm{H}$ & -3.6738747 & -1.1426037 & 4.3655510 \\
\hline $\mathrm{H}$ & -3.5256317 & -0.6561677 & 6.0873177 \\
\hline $\mathrm{C}$ & 0.7795960 & -0.2429860 & 2.4400860 \\
\hline $\mathrm{H}$ & 1.1214535 & 0.5362749 & 1.7431321 \\
\hline $\mathrm{H}$ & 1.6949793 & -0.6795844 & 2.868227 \\
\hline $\mathrm{H}$ & 0.2358358 & -1.0213381 & 1.8915465 \\
\hline $\mathrm{Ca}$ & 10.5062300 & -2.3141500 & -5.6405140 \\
\hline 0 & 12.1714830 & -1.3579430 & -5.575197 \\
\hline O & 9.7713590 & -1.3783080 & -4.129307 \\
\hline
\end{tabular}




\begin{tabular}{|c|c|c|c|}
\hline $\mathrm{C}$ & 12.4272420 & -0.3716520 & -4.8202700 \\
\hline C & 11.5712750 & 0.1439400 & -3.8346470 \\
\hline $\mathrm{H}$ & 11.9130473 & 0.9993832 & -3.2530982 \\
\hline C & 10.3050930 & -0.3924800 & -3.5379560 \\
\hline $\mathrm{C}$ & 13.7563840 & 0.3022280 & -5.0453270 \\
\hline $\mathrm{H}$ & 14.5566078 & -0.4462393 & -4.9382441 \\
\hline $\mathrm{H}$ & 13.9452522 & 1.1426202 & -4.3654760 \\
\hline $\mathrm{H}$ & 13.7969768 & 0.6562343 & -6.0872389 \\
\hline C & 9.4918040 & 0.2429860 & -2.4400860 \\
\hline $\mathrm{H}$ & 9.1499026 & -0.5359315 & -1.7427986 \\
\hline $\mathrm{H}$ & 8.5765557 & 0.6787221 & -2.8694229 \\
\hline $\mathrm{H}$ & 10.0348719 & 1.0220169 & -1.8918530 \\
\hline 0 & 8.8409760 & -3.2703570 & -5.7058310 \\
\hline 0 & 11.2411000 & -3.2499920 & -7.1517200 \\
\hline C & 8.5852180 & -4.2566480 & -6.4607570 \\
\hline C & 9.4411850 & -4.7722400 & -7.4463810 \\
\hline $\mathrm{H}$ & 9.0985593 & -5.6289788 & -8.0255438 \\
\hline C & 10.7073660 & -4.2358200 & -7.7430720 \\
\hline C & 7.2560760 & -4.9305280 & -6.2357010 \\
\hline $\mathrm{H}$ & 6.4539886 & -4.1871433 & -6.3654072 \\
\hline $\mathrm{H}$ & 7.0755232 & -5.7833984 & -6.9021699 \\
\hline $\mathrm{H}$ & 7.2055467 & -5.2642312 & -5.1873344 \\
\hline C & 11.5206550 & -4.8712860 & -8.8409410 \\
\hline $\mathrm{H}$ & 11.7470540 & -4.1056003 & -9.5990381 \\
\hline $\mathrm{H}$ & 12.4874149 & -5.1925626 & -8.423390 \\
\hline $\mathrm{H}$ & 11.0238976 & -5.7252082 & -9.318364 \\
\hline $\mathrm{Ca}$ & 0.2348300 & 2.3141500 & -5.6405140 \\
\hline 0 & 1.9000830 & 3.2703570 & -5.575197 \\
\hline 0 & -0.5000410 & 3.2499920 & -4.1293070 \\
\hline C & 2.1558420 & 66480 & -4.820270 \\
\hline $\mathrm{C}$ & 1.2998750 & 4.7722400 & -3.834647 \\
\hline $\mathrm{H}$ & 1.6479020 & 279823 & -3.2560290 \\
\hline $\mathrm{C}$ & 0.0336930 & 58200 & -3.5379560 \\
\hline C & 3.4849840 & 05280 & -5.0453270 \\
\hline $\mathrm{H}$ & 4.2549904 & 575463 & -5.1790897 \\
\hline $\mathrm{H}$ & 3.7638612 & 98946 & -4.2379482 \\
\hline $\mathrm{H}$ & 3.4364888 & 07039 & -5.9931391 \\
\hline $\mathrm{C}$ & -0.7795960 & 12860 & -2.4400860 \\
\hline $\mathrm{H}$ & -0.8775190 & 09033 & -1.6114686 \\
\hline $\mathrm{H}$ & -1.7965298 & 74167 & -2.8153545 \\
\hline $\mathrm{H}$ & -0.3462020 & 28862 & 576064 \\
\hline 0 & -1.4304240 & 79430 & -5.7058310 \\
\hline O & 0.9697000 & 33080 & 517200 \\
\hline $\mathrm{C}$ & -1.6861820 & 6520 & -6.4607570 \\
\hline $\mathrm{C}$ & -0.8302150 & -0.1439400 & -7.4463810 \\
\hline $\mathrm{H}$ & -1.1960936 & 650153 & -8.0640082 \\
\hline $\mathrm{C}$ & 0.4359660 & 0.3924800 & -7.7430720 \\
\hline $\mathrm{C}$ & -3.0153240 & -0.3022280 & -6.2357010 \\
\hline $\mathrm{H}$ & -3.7752955 & 0.4617814 & -6.023439 \\
\hline $\mathrm{H}$ & -3.3279014 & -0.9391469 & -7.073257 \\
\hline $\mathrm{H}$ & -2.9113374 & -0.9389 & -5.3410436 \\
\hline $\mathrm{C}$ & 1.2492550 & -0.2429860 & -8.840941 \\
\hline $\mathrm{H}$ & 1.7674974 & 0.5429 & -9.406803 \\
\hline $\mathrm{H}$ & 2.0134989 & -0.8753643 & -8.358079 \\
\hline $\mathrm{H}$ & 0.6536 & -0.8768 & -9.510310 \\
\hline $\mathrm{Ca}$ & 10.0365700 & -2.3141500 & 5.640514 \\
\hline 0 & 11.7018240 & -1.3579430 & 5.705831 \\
\hline 0 & 9.3017000 & -1.3783080 & 7.151720 \\
\hline $\mathrm{C}$ & 11.9575820 & -0.3716520 & 6.460757 \\
\hline C & 11.1016150 & 0.1439400 & 7.4463810 \\
\hline $\mathrm{H}$ & 11.4674363 & 0.9650169 & 8.064034 \\
\hline C & 9.8354340 & -0.3924800 & 7.7430720 \\
\hline $\mathrm{C}$ & 13.2867240 & 0.3022280 & 6.2357010 \\
\hline $\mathrm{H}$ & 14.0467748 & -0.4618067 & 6.023877 \\
\hline $\mathrm{H}$ & 13.5990701 & 0.9394626 & 7.0731210 \\
\hline $\mathrm{H}$ & 13.1828173 & 0.9386984 & 5.3408459 \\
\hline C & 9.0221450 & 0.2429860 & 8.8409410 \\
\hline $\mathrm{H}$ & 8.5039895 & -0.5429576 & 9.4069116 \\
\hline $\mathrm{H}$ & 8.2577811 & 0.8753115 & 8.358184 \\
\hline & & 0.7007 & \\
\hline
\end{tabular}




$\begin{array}{rrrr}\mathrm{O} & 8.3713170 & -3.2703570 & 5.5751970 \\ \mathrm{O} & 10.7714410 & -3.2499920 & 4.1293070 \\ \mathrm{C} & 8.1155580 & -4.2566480 & 4.8202700 \\ \mathrm{C} & 8.9715250 & -4.7722400 & 3.8346470 \\ \mathrm{H} & 8.6234414 & -5.6279897 & 3.2560715 \\ \mathrm{C} & 10.2377070 & -4.2358200 & 3.5379560 \\ \mathrm{C} & 6.7864160 & -4.9305280 & 5.0453270 \\ \mathrm{H} & 6.0165871 & -4.1575009 & 5.1799944 \\ \mathrm{H} & 6.5070812 & -5.6192009 & 4.2375220 \\ \mathrm{H} & 6.8351497 & -5.4914943 & 5.9926848 \\ \mathrm{C} & 11.0509960 & -4.8712860 & 2.4400860 \\ \mathrm{H} & 11.1467274 & -4.1517482 & 1.6104868 \\ \mathrm{H} & 12.0685974 & -5.0551880 & 2.8145764 \\ \mathrm{H} & 10.6187247 & -5.8040162 & 2.0590733\end{array}$

